Aftabur Dipu Rahman

\title{
Atmospheric Neutrino Induced Muons in the MINOS Far Detector
}

The Main Injector Neutrino Oscillation Search (MINOS) is a long baseline neutrino oscillation experiment. The MINOS Far Detector, located in the Soudan Underground Laboratory in Soudan MN, has been collecting data since August 2003. The scope of this dissertation involves identifying the atmospheric neutrino induced muons that are created by the neutrinos interacting with the rock surrounding the detector cavern, performing a neutrino oscillation search by measuring the oscillation parameter values of $\Delta m_{23}^{2}$ and $\sin ^{2} 2 \theta_{23}$, and searching for CPT violation by measuring the charge ratio for the atmospheric neutrino induced muons.

A series of selection cuts are applied to the data set in order to extract the neutrino induced muons. As a result, a total of 148 candidate events are selected. The oscillation search is performed by measuring the low to high muon momentum ratio in the data sample and comparing it to the same ratio in the Monte Carlo simulation in the absence of neutrino oscillation. The measured double ratios for the "all events" $(A)$ and high resolution $(H R)$ samples are

$$
R_{A}=\frac{R_{\text {low } / \text { high }}^{\text {data }}}{R_{\text {low } / \text { high }}^{M C}}=0.60_{-0.10}^{+0.11}(\text { stat }) \pm 0.08(\text { syst })
$$

and

$$
R_{H R}=\frac{R_{\text {low } / \text { high }}^{\text {data }}}{R_{\text {low } / \text { high }}^{M C}}=0.58_{-0.11}^{+0.14}(\text { stat }) \pm 0.05(\text { syst }),
$$


respectively. Both event samples show a significant deviation from unity giving a strong indication of neutrino oscillation.

A combined momentum and zenith angle oscillation fit is performed using the method of maximum log-likelihood with a grid search in the parameter space of $\Delta m^{2}$ and $\sin ^{2} 2 \theta$. The best fit point for both event samples occurs at $\Delta m_{23}^{2}=1.3 \times 10^{-3} \mathrm{eV}^{2}$, and $\sin ^{2} 2 \theta_{23}=1$. This result is compatible with previous measurements from the Super Kamiokande experiment and Soudan 2 experiments.

The MINOS Far Detector is the first underground neutrino detector to be able to distinguish the charge of the muons. The measured charge is used to test the rate of the neutrino to the anti-neutrino oscillations by measuring the neutrino induced muon charge ratio. Using the high resolution sample, the $\mu^{+}$to $\mu^{-}$double charge ratio has been determined to be

$$
R_{C P T}=\frac{R_{\mu^{-} / \mu^{+}}^{\text {data }}}{R_{\mu^{-} / \mu^{+}}^{M C}}=0.90_{-0.18}^{+0.24}(\text { stat }) \pm 0.09(\text { syst })
$$

With the uncertainties added in quadrature, the CPT double ratio is consistent with unity showing no indication for $\mathrm{CPT}$ violation. 


\title{
UNIVERSITY OF MINNESOTA
}

This is to certify that I have examined this bound copy of a doctoral thesis by

\begin{abstract}
Aftabur Dipu Rahman
and have found that it is complete and satisfactory in all respects and that any and all revisions required by the final examining committee have been made.
\end{abstract}

Professor Marvin L. Marshak

(Faculty Adviser)

Graduate School 


\title{
Atmospheric Neutrino Induced Muons in the MINOS Far Detector
}

\author{
A THESIS \\ SUBMITTED TO THE FACULTY OF THE GRADUATE SCHOOL \\ OF THE UNIVERSITY OF MINNESOTA \\ BY
}

Aftabur Dipu Rahman

IN PARTIAL FULFILLMENT OF THE REQUIREMENTS

FOR THE DEGREE OF

Doctor Of Philosophy 
(C) Aftabur Dipu Rahman 2007

\section{ALL RIGHTS RESERVED}




\title{
Atmospheric Neutrino Induced Muons in the MINOS Far Detector
}

\author{
by Aftabur Dipu Rahman \\ Under the supervision of Professor Marvin L. Marshak
}

\begin{abstract}
The Main Injector Neutrino Oscillation Search (MINOS) is a long baseline neutrino oscillation experiment. The MINOS Far Detector, located in the Soudan Underground Laboratory in Soudan MN, has been collecting data since August 2003. The scope of this dissertation involves identifying the atmospheric neutrino induced muons that are created by the neutrinos interacting with the rock surrounding the detector cavern, performing a neutrino oscillation search by measuring the oscillation parameter values of $\Delta m_{23}^{2}$ and $\sin ^{2} 2 \theta_{23}$, and searching for CPT violation by measuring the charge ratio for the atmospheric neutrino induced muons.

A series of selection cuts are applied to the data set in order to extract the neutrino induced muons. As a result, a total of 148 candidate events are selected. The oscillation search is performed by measuring the low to high muon momentum ratio in the data sample and comparing it to the same ratio in the Monte Carlo simulation in the absence of neutrino oscillation. The measured double ratios for the "all events" $(A)$ and high resolution $(H R)$ samples are$$
R_{A}=\frac{R_{\text {low } / \text { high }}^{\text {data }}}{R_{\text {low } / \text { high }}^{M C}}=0.60_{-0.10}^{+0.11}(\text { stat }) \pm 0.08(\text { syst })
$$ 
and

$$
R_{H R}=\frac{R_{\text {low } / \text { high }}^{\text {data }}}{R_{\text {low } / \text { high }}^{M C}}=0.58_{-0.11}^{+0.14}(\text { stat }) \pm 0.05(\text { syst }),
$$

respectively. Both event samples show a significant deviation from unity giving a strong indication of neutrino oscillation.

A combined momentum and zenith angle oscillation fit is performed using the method of maximum log-likelihood with a grid search in the parameter space of $\Delta m^{2}$ and $\sin ^{2} 2 \theta$. The best fit point for both event samples occurs at $\Delta m_{23}^{2}=1.3 \times 10^{-3} \mathrm{eV}^{2}$, and $\sin ^{2} 2 \theta_{23}=1$. This result is compatible with previous measurements from the Super Kamiokande experiment and Soudan 2 experiments.

The MINOS Far Detector is the first underground neutrino detector to be able to distinguish the charge of the muons. The measured charge is used to test the rate of the neutrino to the anti-neutrino oscillations by measuring the neutrino induced muon charge ratio. Using the high resolution sample, the $\mu^{+}$to $\mu^{-}$double charge ratio has been determined to be

$$
R_{C P T}=\frac{R_{\mu^{-} / \mu^{+}}^{d a t a}}{R_{\mu^{-} / \mu^{+}}^{M C}}=0.90_{-0.18}^{+0.24}(\text { stat }) \pm 0.09(\text { syst }) .
$$

With the uncertainties added in quadrature, the CPT double ratio is consistent with unity showing no indication for CPT violation. 


\section{Acknowledgements}

First of all, I would like to thank my adviser Professor Marvin Marshak for all the support he has give me for the last six years. His words of encouragement all those years ago, telling me not to quit graduate school when I was ready to give up, meant a lot. I am grateful that he has allowed me to work at my own pace. I am also grateful that he has given me the "push" when needed it.

I would also like to thank Dr. Peter Litchfield for overseeing all my work for the last ten months. His open door policy and friendly attitude was always appreciated. I greatly admire his patience and good advices. I could not have finished the dissertation in time if it wasn't for Peter. Thanks to all my committee members, professor Hans Courant, Professor Michal Voloshin, and Professor Bob Gerhz for reading the thesis, and passing me.

It gives me great pleasure to thank my girlfriend, Laurel Johnson. I cannot thank her enough for being my friend through the whole process. Laurel has been as kind as she has been patient, and as loving as she has been supportive. She has kept me sane with her 
words of encouragement in times of crisis. Having you in my life means so much! Thank you!

A special thank you goes to the Wayne and Ann Johnson, Laurel's parents. I have thoroughly enjoyed spending time with you and getting to know you. Thank you for bringing up a wonderful daughter and for being wonderful people! Thanks to Beck Johnson, Jim, Sharon, Emily and Jordan Eaton, Joe, Janet, Alex and George, and the rest of the Johnson family for giving me the family support I needed.

For the last several years many people have become important in my life. Their support and friendship will never be forgotten. I have known Rashed Ahmed since we were in middle school. He has been a dear friend to me. Rashed's practical advice and support was always appreciated. Thanks to Steve Giron for being my friend, mentor and caring for me like a brother. Thanks to Jessica Shryack for giving me the space I needed in the last couple of months. Thank you for your understanding. Thanks to Mike Matney for having faith in me all these years. Thanks to Kim and Devin. Thanks to Long Duong, Sharanya Udipi, Mike Myhrom, Siri, Scott Tretter, Joe Quam, Alexia Nompeli, Laura Smith and Esther Chong for being wonderful friends and enriching my life. Thanks to Toni Schwarz, Jenell Wilmot, Ryan, Anne, Janice Bisch, Irina Raskin, Niko Salgado, and April Haven, Bryan Kuzel, Ann-Sophie, Tim and Bridget Deen, and Monika Kumar. Thanks to Eric Wornson, Justin Pyon, Mickey Arora, Hari Arimilli, and Jake for being my tennis partners and friends.

I would also like to acknowledge the people I have known and cared about during my 
time in undergraduate and graduate school. Thanks to Terri Markos and CK Kwai for being my mentors and great bosses to work for. Thanks to Mary at the International Office for being a sweetheart. Thanks to my physics professors Fred Otto, Dan Bloom, and Richard Shields for being excellent teachers and advisers at Winona State University. Thanks to my graduate school professors Earl Peterson, Keith Ruddick, Ken Heller, Yuichi Kubota, Ron Polling, Jon Urheim, Chuck Campbell and Serge Rudaz. Thanks to all the post-docs from whom I have learned a lot. Thanks to Sue Kasahara for patiently helping me with programming. Thanks to David Petyt, Leon Mualem, Kregg Arms, and Pete Border. Thanks to Bobbi Eich, Judy Sione, Joyce Soine and Dave Holets for all the paper work help as well as keeping me out of trouble. I would also like to thank my officemates. A special thank you goes to Ben Speakman and Jeremy Gogos. Both Ben and Jeremy have given a tremendous amount of help with programming. Without their support I would not have finished in time. Thanks to Sujeewa Kumaratunga, Eric Grashorn, Erik Beall and Bernie Becker for putting up with me in the office. Thanks to Louie Barrett, Bill Miller, Jerry Meier and Eileen Amos at the Soudan Underground Laboratory for providing an excellent working atmosphere. Thanks to Brian Rebel for providing the framework for the thesis and answering any questions I had. Thanks to all my friends at the MINOS collaboration; Andy Blake, Leo Jenner, Caius Howcroft, Alex Sousa and Jeff Hartnell.

I would like to acknowledge family. Thanks to my grandfather for raising the Rahmans with integrity. Thanks to all my aunts and uncles, and cousins for the love they have given me. Thanks to my brother, Tipu Rahman, for being there for me in the last ten years. 
Finally, I would like to say thank you to my parents. I am certain that words will not justify my gratitude towards them. They have sacrificed their entire life for my brother and I. I thank my father, Commodore Mafizur Rahman, for being a role model and showing an incredible amount of support for the last ten years. I thank my mother, Dr. Anjuman Ara Rahman, for the care, love and kindness she has given me. To my abbu and ammu, I dedicate this dissertation in your honor. 


\section{Dedication}

This dissertation is dedicated to my loving parents. 


\section{Contents}

Abstract i

Acknowledgements

Dedication v v

List of Tables $\quad x$

List of Figures $\quad$ xi

1 Introduction 1

$\begin{array}{lll}2 & \text { Theorv of Neutrino Oscillations } & 7\end{array}$

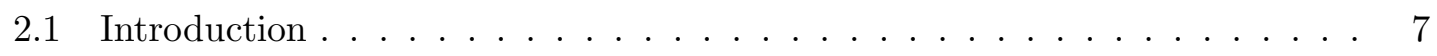

2.2 Neutrino Oscillations in Vacuum $\ldots \ldots \ldots \ldots$

2.2.1 Three Flavor Mixing . . . . . . . . . . . . . . . . 8

2.2 .2 Two Flavor Mixing . . . . . . . . . . . . . . . . . . . . . . . . . 10

2.3 Neutrino Oscillations in Matter . . . . . . . . . . . . . . 12 
3 Previous Measurements

3.1 Introduction . . . . . . . . . . . . . . . . . . . 18

3.2 The Super-Kamiokande Experiment . . . . . . . . . . . . . . . . . . 20

3.3 The Soudan 2 experiment . . . . . . . . . . . . . . . . . . . . . . 24

4 The MINOS Far Detector $\quad 28$

4.1 Introduction . . . . . . . . . . . . . . . . . . . 28

4.2 General Overview . . . . . . . . . . . . . . . . . . . . . . . . 29

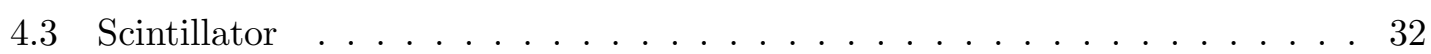

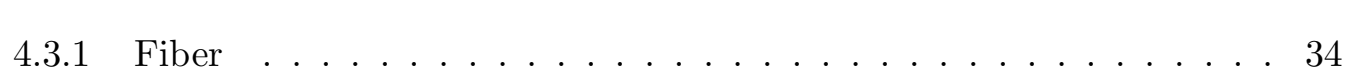

4.4 Photomultiplier Tubes (PMT) . . . . . . . . . . . . . . . . . . . 35

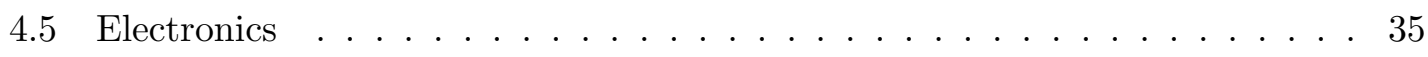

4.6 Data Acauisition Svstem (DAQ) $\ldots \ldots$. . . . . . . . . . . . . . 37

4.7 Phvsics Event Information . . . . . . . . . . . . . . . . . . 38

4.8 Magnetics $\ldots \ldots \ldots \ldots \ldots$. . . . . . . . . . . . . . . . . . . 40

4.9 Calibration . . . . . . . . . . . . . . . . . . . . 41

5 Cosmic Rav Muons and Atmospheric Neutrinos 43

5.1 Introduction . . . . . . . . . . . . . . . . . 43

5.2 Cosmic Rav Muons . . . . . . . . . . . . . . . . . . . . . . 44

5.2 .1 Cosmic Rav Flux . . . . . . . . . . . . . . . . . 44

5.2 .2 Simulation of Underground Muons . . . . . . . . . . . . . . 47 
5.2 .3 Data to $\mathrm{MC}$ comparison . . . . . . . . . . . . . . . . 49

5.3 Atmospheric Neutrinos . . . . . . . . . . . . . . . . . 53

5.3 .1 Atmospheric Neutrino Flux ... . . . . . . . . . . . . 53

5.3.2 Uncertainties in the Flux Calculations . . . . . . . . . . . . . 57

5.3.3 Simulation of the Neutrino Induced Muons . . . . . . . . . . . . 58

5.3 .4 Neutrino Cross Sections . . . . . . . . . . . . . . . 60

6 Data Selection $\quad 63$

6.1 Introduction . . . . . . . . . . . . . . . . . 63

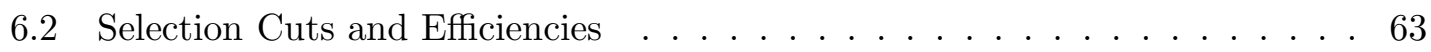

6.2 .1 Track Level Cuts . . . . . . . . . . . . . . . . . . 66

6.2 .2 Strip Level Cuts . . . . . . . . . . . . . . . . . . 72

6.3 Neutrino Induced Candidate Events $\ldots \ldots \ldots$. . . . . . . . . . 77

6.3.1 Upward Going Events . . . . . . . . . . . . . . . . . . . 77

6.3.2 Horizontal Events . . . . . . . . . . . . . . . . . . 81

6.3.3 Charge/Momentum Selection . . . . . . . . . . . . . . . 84

6.3.4 Event Distributions Summarv . . . . . . . . . . . . . . 85

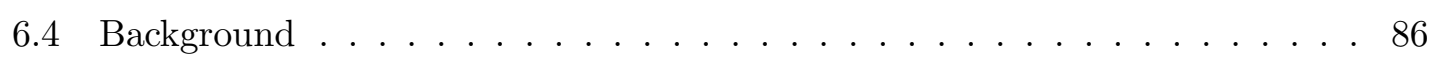

6.4.1 Cosmic Rav Muons . . . . . . . . . . . . . . . . . 86

6.4.2 Contained Upward Going Muons . . . . . . . . . . . . . . 88

6.5 Svstematic Uncertainties . . . . . . . . . . . . . . . . . . 90 


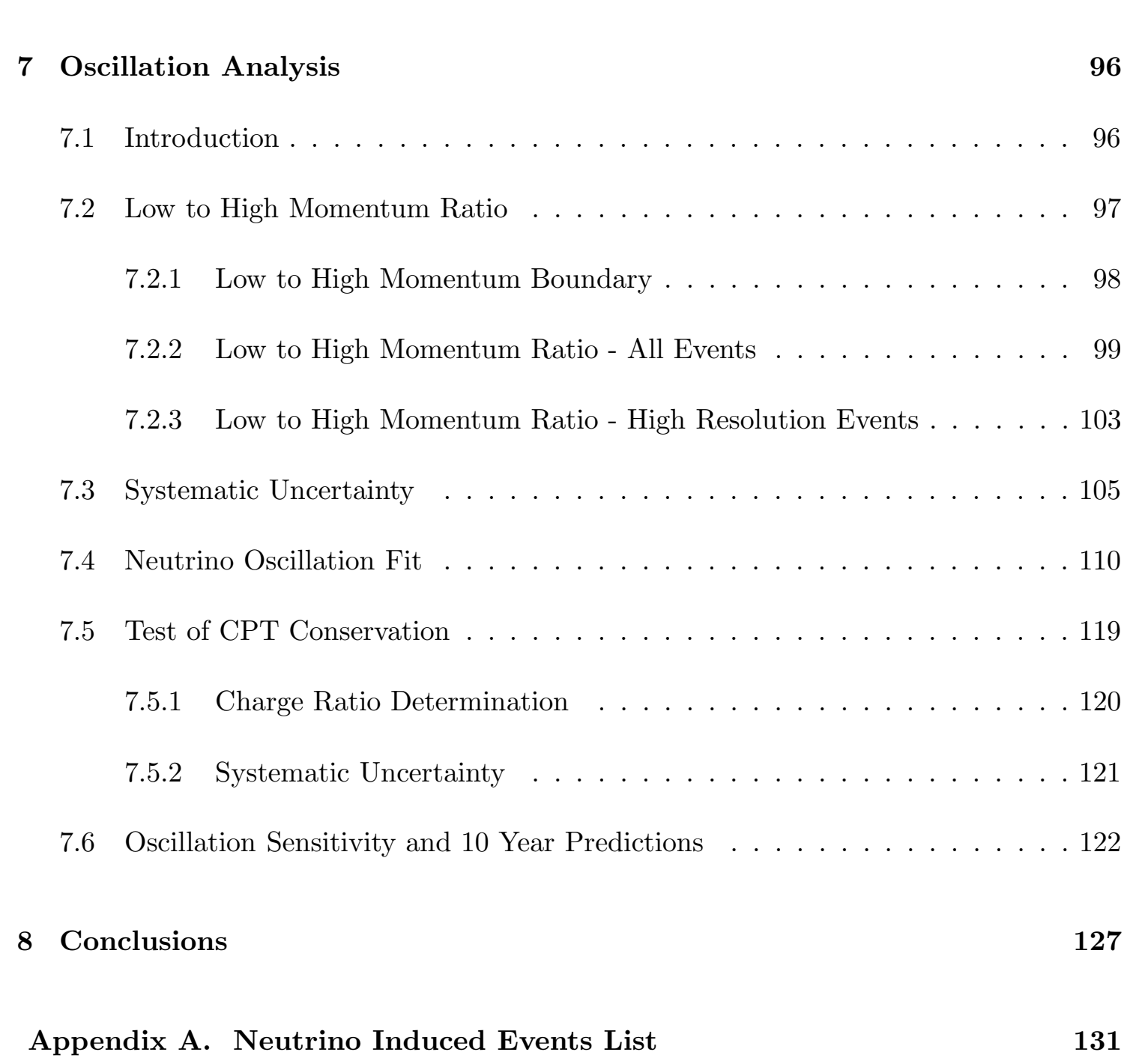




\section{List of Tables}

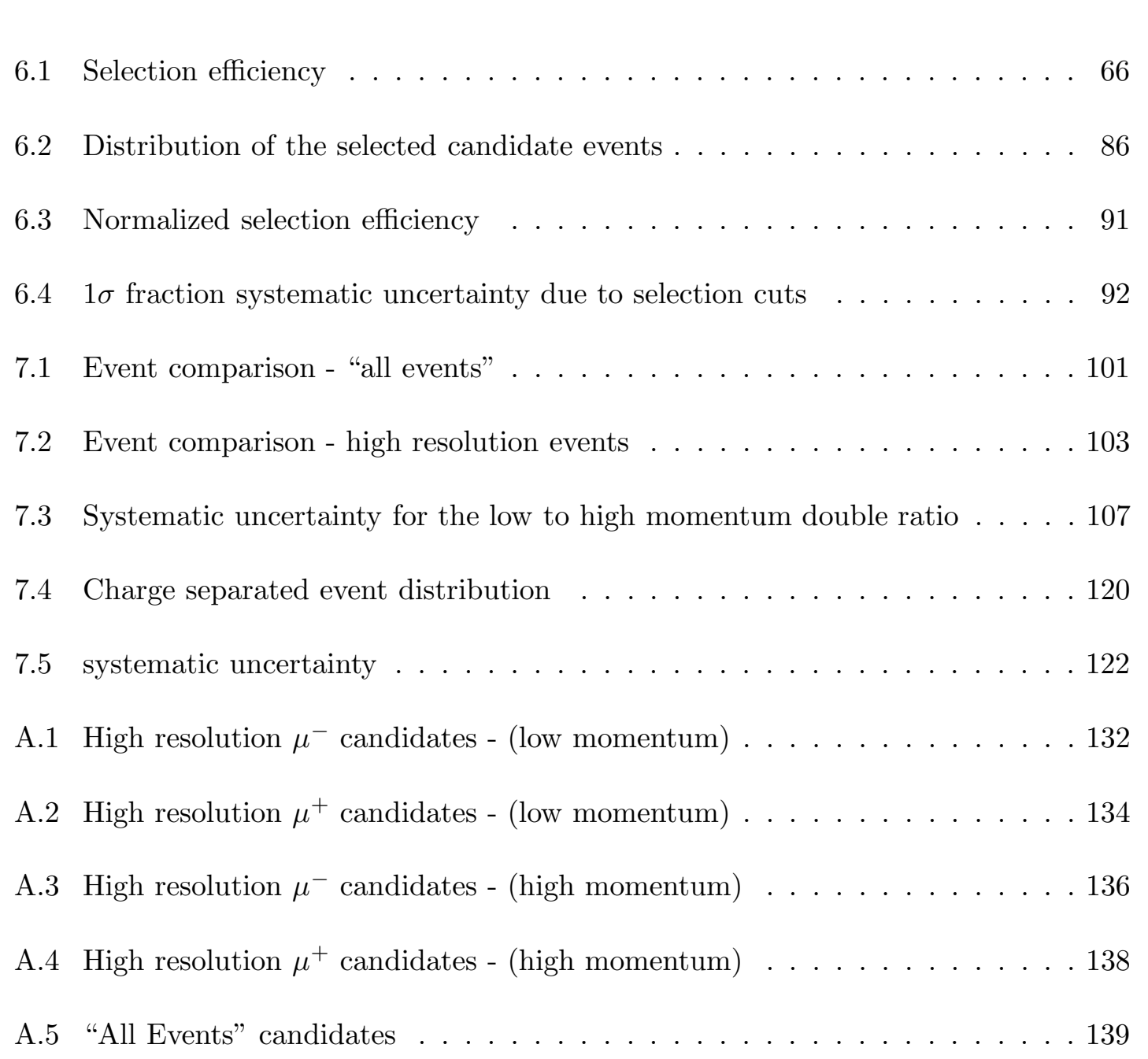




\section{List of Figures}

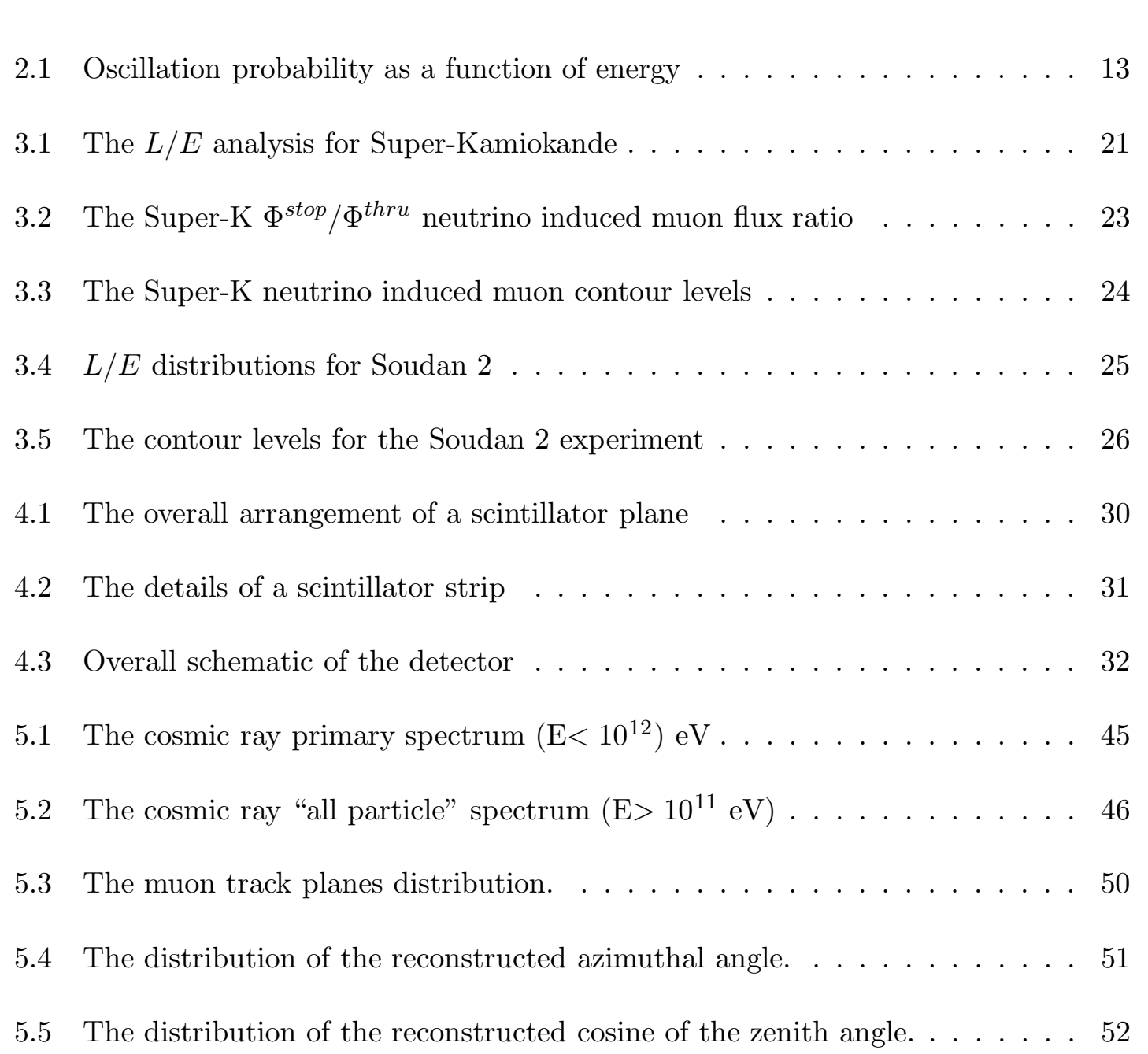


5.6 The flux contribution from the parent mesons . . . . . . . . . 56

5.7 The observed flux for the proton and helium cosmic rav primaries . . . . 58

5.8 Simulated parent neutrino energies $\ldots \ldots \ldots \ldots \ldots \ldots$. . . . . . . . . 59

5.9 Neutrino cross sections . . . . . . . . . . . . . . . . . . 61

5.10 Neutrino cross section ratios . . . . . . . . . . . . . . . . 62

$6.11 / \beta$ distribution for the events passing the track level cuts . . . . . . . . 69

6.2 Track fit $\chi^{2} /$ n.d.f. distributions for the data and the MC . . . . . . . 71

6.3 Time vs. Y scatter plot for two different events. . . . . . . . . . . . 73

6.4 The reconstructed and the re-calculated track fit $\chi^{2} / n d f \mid \ldots \ldots$. . . . 75

6.5 The difference between the spill trigger time and the event vertex time . . . 76

6.6 The inverse $\beta$ distribution for all selected muons $\ldots \ldots \ldots \ldots$

6.7 Candidate upward going muon event . . . . . . . . . . . . . . 80

6.8 The vertex $y$ direction cosine for data $\ldots \ldots \ldots$. . . . . . . . . 82

6.9 Candidate horizontal muon event .................... 83

6.10 Binned charge/momentum purity as a function of $\mid p / \delta p\rfloor \ldots$. . . . . . 84

6.11 Vertex direction cosine and the figure of merit . . . . . . . . . 87

6.12 The radial vertex distribution for the contained atmospheric MC . . . 89

6.13 The radial vertex distribution for the data . . . . . . . . . . . . 90

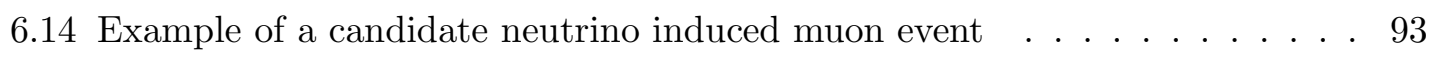

6.15 Example of a candidate neutrino induced muon event . . . . . . . . . 94

6.16 Example of a candidate neutrino induced muon event . . . . . . . . . 95 
7.1 the parent neutrino energy distribution from the MC sample . . . . . . . 97

7.2 MC double ratios as a function of the low/high momentum cut-off _. . . . . 99

7.3 The statistical significance in low/high momentum boundarv selection . . . 100

7.4 Calculation of Poisson confidence limits . . . . . . . . . . . . . . . . . 102

7.5 The momentum separated zenith angle distributions . . . . . . . . . 112

7.6 The $68 \%$ and the $90 \%$ log-likelihood contours . . . . . . . . . . . 113

7.7 The contour levels from the MINOS contained atmospheric analvsis . . . . 115

7.8 The low to high momentum ratio sensitivity in MC . . . . . . . . 116

7.9 The log-likelihood contour plots extended into the unphvsical region. . . . . 118

7.10 The $68 \%$ and the $90 \%$ oscillation sensitivity contours . . . . . . . . . . 124

7.11 Projected 10 year oscillation sensitivity contours $\ldots . .125$ 


\section{Chapter 1}

\section{Introduction}

The "Standard Model" of elementary particle physics describes the current status of knowledge about the basic building blocks of matter and their fundamental interactions. According to the Standard Model, all known matter consists of 12 elementary particles-6 particles called quarks and 6 particles called leptons (from the Greek for "light particles"). The 6 quarks consist of three "families" of two quarks each, called "up" and "down", "strange" and "charmed", and "top" and "bottom." The 6 leptons also consist of three families, each of which consists of a charged lepton and an uncharged lepton. The charged leptons are the "electron," the "muon" and the "tau." The uncharged leptons are known as "neutrinos" and, in all, there are three neutrinos, one associated with each of the charged leptons.

According to the Standard Model, the 12 elementary particles interact with each other 
through four fundamental forces or interactions. The intrinsic strength of these interactions varies by an overall factor of about $10^{40}$. The strongest of these interactions is known simply as the strong (or nuclear interaction). The strong interaction involves only the quarks and not the leptons. It holds the nuclei of atoms together and is responsible for the existence of the 92 naturally stable elements. The next strongest force is electromagnetism, which is about two orders of magnitude weaker than the strong interaction. Electromagnetism only affects particles with electric charge. Thus, electromagnetic interactions affect the 6 quarks and the three charged leptons, but not the uncharged leptons known as neutrinos. All 12 elementary particles are affected by the weak interaction, which is about 11 orders of magnitude weaker than the strong interaction. The weak interaction is mediated by the massive $\mathrm{W}$ and $\mathrm{Z}$ bosons and is thus a short range force. The weak interaction is unique, in that it is the only fundamental interaction capable of changing flavor. The weak interaction also violates both parity $(\mathrm{P})$ conservation and charge conjugation-parity (CP) conservation. The weak interaction only affects particles with left-handed helicity and all known neutrinos are believed to be left-handed. The final fundamental interaction is gravity, which is about 38 orders of magnitude weaker than the strong interaction. Despite its intrinsic weakness, gravity is the most macroscopically visible force because it affects all mass and energy, is always attractive and has a long range. Several large interferometric experiments are currently seeking more information about gravity that might be useful towards the formulation of a consistent model of quantum gravity. 
Neutrinos have played a fascinating role in the history of the physics of elementary particles. The existence of neutrinos was first hypothesized by the Austrian physicist Wolfgang Pauli in 1930 as an explanation for the observed energy, momentum and angular momentum non-conservation in radioactive beta decay, the processes in which a neutron decays into a proton and a electron. The electron in beta decay has a continuous energy spectrum, while a two-body decay should result in a well-defined electron energy. Although Pauli initially named his particle as neutronen in German, the discovery of the stronglyinteracting neutron by James Chadwick led Enrico Fermi to rename Pauli's particle as a "neutrino," using the Italian diminutive suffix, when Fermi published his theory of the weak interactions in 1933.

The neutrino was first observed by Fred Reines and Clyde Cowan in 1956 at the Savannah River Reactor in South Carolina after an initial, inconclusive experiment at the Hanford Reactor in Washington. The detection was by the inverse beta decay reaction in which an anti-neutrino from the reactor combined with a proton in the $\mathrm{CdCl}_{2}$ aqueous solution target to produce a neutron and a positron. The positron was detected through the annihilation reaction that produces two back-to-back $0.5 \mathrm{MeV} \gamma$ rays. The neutron was slowed by the water and then captured by a cadmium atom resulting in the delayed emission of several gamma rays. Reines received the Nobel Prize in 1995 for this work.

Subsequent experiments following the discovery of the neutrino continued to yield surprising results. Leon Lederman, Mel Schwartz and Jack Steinberger were awarded the Nobel Prize in 1988 for their discovery of the neutrino associated with the muon in the 
1960's. The electroweak unification model, also developed in the 1960's, earned the Nobel Prize for Sheldon Glashow, Abdus Salam and Steven Weinberg in 1979. It's first verified prediction was the existence of neutral current neutrino nucleon scattering, whereas the only previously known process was charged current scattering. Neutral current neutrino scattering was first observed in a bubble chamber experiment at CERN during the early 1970's, which prepared the path for the later discovery in the early 1980's, also at CERN, of the $\mathrm{W}$ and $\mathrm{Z}$ bosons as the carriers of the weak force. Neutral current scattering is associated with the neutral $\mathrm{Z}$ boson, while charged current scattering is associated with the charged $\mathrm{W}$ boson.

Other surprises in the neutrino sector were the results of radiochemical, deep underground solar neutrino studies. The first such experiment was initiated by Ray Davis in the Homestake Mine in Lead, SD in the late 1960's. The experiment was originally conceived as a way to probe processes in the solar core using neutrinos as a probe because, unlike all other particles, they could easily travel directly through the sun. Davis installed a tank with approximately 600 tons of perchloroethylene about $1.5 \mathrm{~km}$ underground. The goal of the experiment was to detect neutrinos through the inverse beta decay reaction that transforms ${ }^{37} \mathrm{Cl}$ to ${ }^{37} \mathrm{Ar}$ and then to detect argon by counting its radioactive decays. Both the Davis experiment and later radiochemical experiments using gallium detected solar neutrino fluxes significantly lower than predicted by standard solar models. Because of the difficult nature of these experiments and uncertainties regarding the solar models, the Davis and later experiments were not taken seriously by many observers for a number of 
years. However, by the early 1990's, it became clear that a significant fraction of the solar neutrino flux disappeared between the Sun and the Earth. Davis received the Nobel Prize in 2002 for his pioneering contributions to the detection of cosmic neutrinos.

In the early 1980's, a series of experiments began searching for proton decay, the spontaneous conversion of matter into energy by the spontaneous disintegration of protons and bound neutrons. The success of electroweak unification spurred the development of the so-called "Grand Unified Theories" or "GUTS" during the late 1970's. These theories unified the strong, electromagnetic and weak interactions and thus generally connected quarks and leptons, leading to a probability for quark disappearance and lepton appearance. The initial estimates for the proton lifetime, using a particularly simple symmetry known as $\mathrm{SU}(5)$, was about $10^{30}$ years. The search for proton decay thus required a multi-kiloton detector instrumented sufficiently to recognize the energy and momentum of a single nucleon decay. About a half-dozen such detectors were built in various laboratories around the world, all of which were located deep underground in order to shield the detectors from cosmic rays.

The most significant expected background to the search for proton decay was cosmic ray neutrino interactions in the deep underground detectors. For this reason, physicists searching for proton decay carefully studied neutrino interactions in their detectors, in order to be sure that they could uniquely identify a nucleon decay, if one occurred. While no uniquely identifiable proton decays were ever observed, most proton decay experiments 
measured a deficit of muon-type cosmic ray neutrinos compared to the number of electrontype cosmic ray neutrinos. By the middle 1990's, there was significant evidence pointing towards the existence of spontaneous flavor changes during neutrino propagation, which resulted in measurable deficits for both the solar electron-type neutrinos and the cosmic ray atmospheric muon-type neutrinos. This flavor-changing process is now known as "neutrino oscillations" and is the subject of the research described in this dissertation. 


\section{Chapter 2}

\section{Theory of Neutrino Oscillations}

\section{$2.1 \quad$ Introduction}

The phenomenon of neutrino oscillations is the first observation of elementary particle physics beyond the Standard Model (SM). The SM predicts massless neutrinos, similar to the photon and in accordance with Pauli's initial hypothesis. Neutrino oscillation is a model motivated by observation, namely the disappearance of electron-type solar neutrinos and muon-type atmospheric neutrinos. The essence of the model is the hypothesis that neutrinos have mass and that the mass eigenstates and the flavor eigenstates are not identical. The oscillation phenomenon can be observed by preparing a beam of neutrinos in flavor eigenstates by pion or kaon decay. The beam neutrinos of energy $E$ are allowed to propagate a distance $L$. This propagation "analyzes" the neutrinos into mass eigenstates. The neutrinos are then re-analyzed into flavor eigenstates by observing their 
charged current interactions in a suitable detector. Because the mass eigenstates are a linear superposition of the flavor eigenstates, even a initially single-flavor neutrino beam propagates as three different mass neutrinos. The superposition of these mass eigenstates into flavor eigenstates at a far detector leads to the possible detection of all three neutrino flavors, even if the initial beam had only a single flavor. The degree to which the initial flavor is depleted and the other flavors populated depends on the neutrino energy, the length of the beam and the square of the mass difference between the neutrino states. There is also an additional parameter that indicates the degree to which this mixing process affects the neutrinos. In a two-neutrino oscillation scenario, these parameters are known as $\Delta m_{23}^{2}$ and $\sin ^{2} 2 \theta_{23}$.

\subsection{Neutrino Oscillations in Vacuum}

\subsubsection{Three Flavor Mixing}

The neutrino flavor eigenstates can be written as linear combination mass eigenstates assuming they have non-zero Dirac mass:

$$
\nu_{\alpha}=\sum_{i} U_{\alpha i} \nu_{i}
$$

here $\alpha$ denotes the three flavor eigenstates $\left(\nu_{e}, \nu_{\mu}\right.$ and $\left.\nu_{\tau}\right)$, and $i$ denotes the mass eigenstates $\left(\nu_{1}, \nu_{2}\right.$ and $\left.\nu_{3}\right)$. U is the unitary Maki-Nakagawa-Sakata (MNS) lepton mixing matrix [1]. U can be parameterized as in the quark Cabibbo-Kobayashi-Maskawa (CKM) 
mixing matrix:

$$
\left(\begin{array}{c}
\nu_{e} \\
\nu_{\mu} \\
\nu_{\tau}
\end{array}\right)=\left(\begin{array}{ccc}
c_{1} & s_{1} c_{3} & s_{1} s_{3} \\
-s_{1} c_{2} & c_{1} c_{2} c_{3}-s_{2} s_{3} e^{i \delta} & c_{1} c_{2} s_{3}+s_{2} c_{3} e^{i \delta} \\
-s_{1} s_{2} & c_{1} s_{2} s_{3}+c_{2} s_{3} e^{i \delta} & c_{1} s_{2} s_{3}-c_{2} c_{3} e^{i \delta}
\end{array}\right)\left(\begin{array}{c}
\nu_{1} \\
\nu_{2} \\
\nu_{3}
\end{array}\right)
$$

where $c_{i}=\cos \theta_{i}, s_{i}=\sin \theta_{i}, \theta_{i}$ are the weak mixing angles and $e^{i \delta}$ is a complex phase. The initial state, at time $t=0$ for $\nu_{\alpha}$, using the Einstein summation convention is:

$$
\left|\nu_{\alpha}(0)\right\rangle=U_{i}\left|\nu_{i}\right\rangle
$$

The mass eigenstates for the neutrinos with energy $E_{i}$ and momentum $p$, are given by:

$$
E^{2}=p^{2}+m_{i}^{2}
$$

The time evolution which are dependent on energy eigenvalues is described by:

$$
\left|\nu_{\alpha}(t)\right\rangle=U_{\alpha i} e^{i E_{i} t}\left|\nu_{i}\right\rangle
$$

One can now state that the probability of neutrino being in $\nu_{\beta}$ at a later time $t$ is:

$$
\begin{aligned}
P\left(\nu_{\alpha} \rightarrow \nu_{\beta}\right) & =\left|\left\langle\nu_{\beta} \mid \nu_{\alpha}(t)\right\rangle\right|^{2} \\
& =\left|\left\langle U_{\beta} i \nu_{i} \mid U_{\alpha} i e^{-i E_{i} t} \nu_{i}\right\rangle\right|^{2}
\end{aligned}
$$




$$
=\sum_{i}\left|U_{\alpha} i\right|^{2}\left|U_{\beta} i\right|^{2}+\sum_{i \neq j} U_{\alpha i} U_{\beta i}^{\star} U_{\alpha j}^{\star} U_{\beta j} e^{-i\left(E_{i}-E_{j}\right) t}
$$

The derivation of the disappearance probability of $\nu_{\alpha} \rightarrow \nu_{\beta}$ can also be achieved for Majorana mass terms. However, one has to take into account that the neutrinos can also oscillate to anti-neutrinos and the derivations become more complicated and hence it is left out.

\subsubsection{Two Flavor Mixing}

Although we expect that all the three flavors of neutrinos mix, there are, however, dominant modes for the three flavors. The probability of two neutrino oscillation, where two mass eigenstates and two flavors are relevant, can be approximated. The unitary matrix $\mathrm{U}$, when two mass eigenstates and one mass difference splitting $\left(\Delta m^{2}\right)$ are relevant, takes the form [2]:

$$
\left(\begin{array}{c}
\nu_{\alpha} \\
\nu_{\beta}
\end{array}\right)=\left(\begin{array}{cc}
\cos \theta & \sin \theta \\
-\sin \theta & \cos \theta
\end{array}\right)\left(\begin{array}{l}
\nu_{1} \\
\nu_{2}
\end{array}\right)
$$

where $\theta$ is the weak mixing angle between flavor and mass eigenstates. Taking $\alpha$ and $\beta$ to be $\nu_{e}$ and $\nu_{\mu}$ respectively, the probability of two flavor oscillation $\left(\nu_{\nu} \rightarrow \nu_{e}\right)$ can now be calculated. The time evolution equation is described by,

$$
\left|\nu_{\mu}(t)\right\rangle=\cos \theta e^{-i E_{2} t}\left|\nu_{2}\right\rangle-\sin \theta e^{-i E_{2} t}\left|\nu_{1}\right\rangle
$$


Hence the oscillation probability is given by:

$$
\begin{aligned}
P\left(\nu_{\mu} \rightarrow \nu_{e}\right) & =\left|\left\langle\nu_{\beta} \mid \nu_{\alpha}(t)\right\rangle\right|^{2} \\
& =\sin ^{2} \theta \cos ^{2} \theta\left(2-e^{-i\left(E_{1}-E_{2}\right) t}-e^{i\left(E_{1}-E_{2}\right) t}\right) \\
& =2 \sin ^{2} \theta \cos ^{2} \theta\left(1-\cos \left(\left(E_{1}-E_{2}\right) t\right)\right)
\end{aligned}
$$

Assuming $m_{i} \ll p$, Equation 2.4 yields:

$$
\begin{aligned}
E_{i} & =\sqrt{p^{2}+m_{i}^{2}} \\
& \approx p+\frac{m_{i}^{2}}{2 p}
\end{aligned}
$$

therefore:

$$
\begin{aligned}
E_{1}-E_{2} & \approx \frac{m_{1}^{2}-m_{2}^{2}}{2 p} \\
& \approx \frac{\Delta m_{12}^{2}}{2 p}
\end{aligned}
$$

where $\Delta m_{12}^{2}=m_{1}^{2}-m_{2}^{2}$. If the neutrinos travel a distance L, and substituting $L$ for $t$ (ultrarelativistic neutrinos), we can get the following result using natural units $(c=1, \hbar=1)$ :

$$
P\left(\nu_{\mu} \rightarrow \nu_{e}\right)=\sin ^{2} 2 \theta \sin ^{2} \theta\left(\frac{\Delta m^{2} L}{4 p}\right)
$$


It is useful at this point to develop a system of units where energy is in $\mathrm{GeV}$, mass is in $\mathrm{eV}$ and length is in kilometers. After reinserting the proper factors of $c$ and $\hbar$, we get the familiar expression for two flavor neutrino oscillation in vacuum,

$$
P\left(\nu_{\mu} \rightarrow \nu_{e}\right)=\sin ^{2} 2 \theta_{13} \sin ^{2}\left(\frac{1.27 \Delta m_{13}^{2} L}{E}\right)
$$

Similarly, the dominant two flavor oscillation mode $\nu_{\mu} \rightarrow \nu_{\tau}$ is given by replacing the mass eigenstate subscripts from " 13 " to " 23 ",

$$
P\left(\nu_{\mu} \rightarrow \nu_{\tau}\right)=\sin ^{2} 2 \theta_{23} \sin ^{2}\left(\frac{1.27 \Delta m_{23}^{2} L}{E}\right) .
$$

The survival probability $P_{s}\left(\nu_{\mu} \rightarrow \nu_{\mu}\right)$ is given by,

$$
P_{s}\left(\nu_{\mu} \rightarrow \nu_{\mu}\right)=1-P\left(\nu_{\mu} \rightarrow \nu_{\tau}\right)
$$

Figure 2.1 shows the oscillation probability as a function of the neutrino energy for different values of neutrino baseline using the Super Kamiokande best fit parameters [18, where $\Delta m^{2}=2.4 \times 10^{-3} \mathrm{eV}^{2}$ and $\sin ^{2} 2 \theta=1$.

\subsection{Neutrino Oscillations in Matter}

There are significant differences between neutrino oscillations in vacuum and neutrino oscillations in matter. The most illuminating is the resonant enhancement of probability 

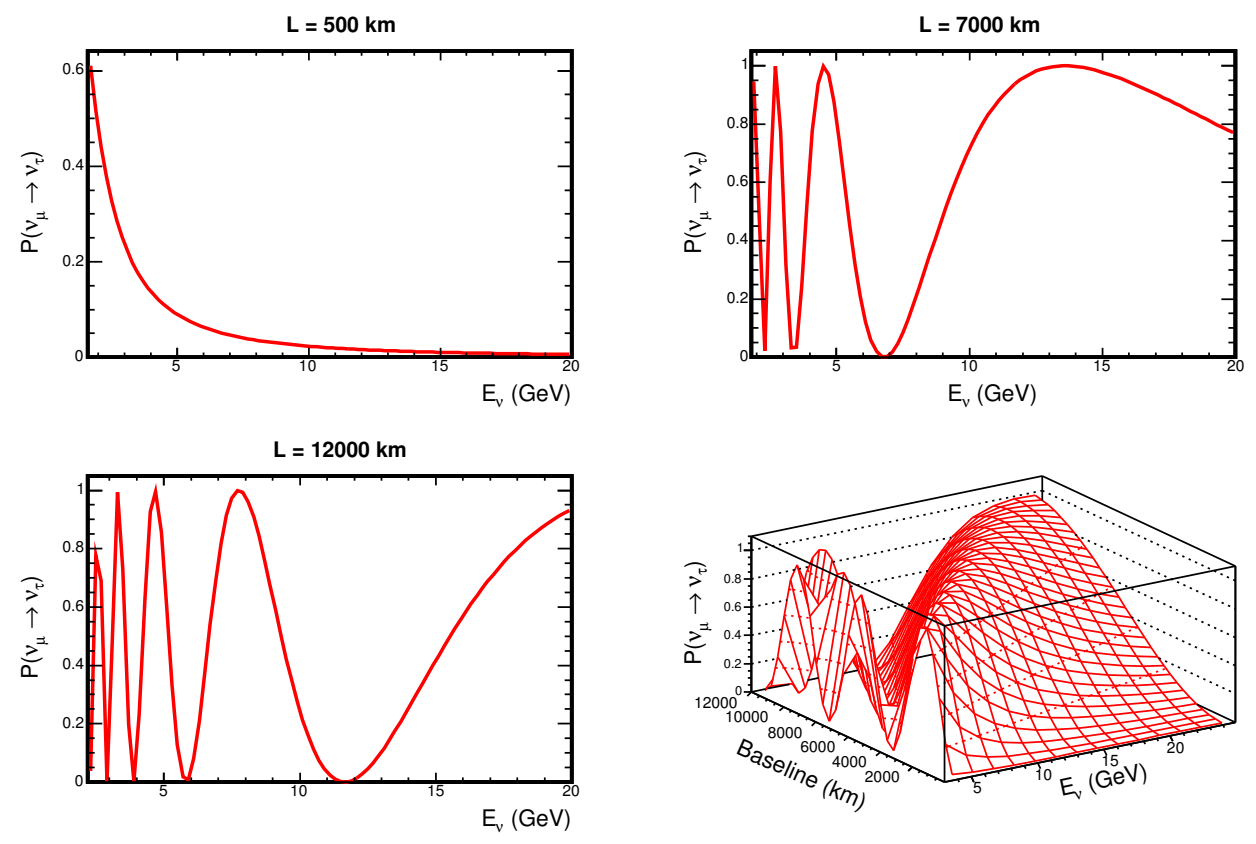

Figure 2.1: Oscillation probability as a function of energy for 3 different neutrino baselines, where $\mathrm{L}=500 \mathrm{~km}$ represents neutrinos coming from the horizon $\left(\theta_{\text {zenith }}=90^{\circ}\right)$, and $\mathrm{L}$ $=12000 \mathrm{~km}$ represents neutrinos that are produced on the other side of the Earth's atmosphere $\left(\theta_{\text {zenith }}=180^{\circ}\right)$. The bottom right plot shows the oscillation probability as a function of the neutrino baseline and energy. The oscillation parameters used were taken from the Super Kamiokande best fit result [18].

described by Mikheyev [3], Smirnov, and Wolfenstein [4], known as the MSW effect. Matter can enhance neutrino mixing. The probabilities for neutrino flavor change can be large despite the smallness of the vacuum mixing angle.

Neutrinos traveling through matter will interact with it once in a great while. The probability for the interaction, being proportional to the square of the Fermi constant, is very small. However, the probability goes up if the matter number density is high, for example, on the sun and maybe on Earth. Neutrinos in matter can undergo forward 
scattering where the momentum is unaltered. This scattering process sets up a mean potential, $V_{a}$, that is proportional to the matter number density. When this induced potential is comparable to the neutrino kinetic energy difference $\Delta m^{2} / 2 E$, the matter effect can strongly enhance neutrino oscillation.

All three types of neutrinos interact with matter through the neutral current (NC) interaction by exchanging a $Z^{0}$ boson. Only the electron interacts with matter through charged current (CC) interaction by exchanging a $W^{ \pm}$boson. At relatively low energies the potential for a $\mathrm{CC}$ interaction is

$$
\left(V_{\nu_{e}}\right)_{C C}=\sqrt{2} G_{F} N_{e}
$$

where $G_{F}$ is the Fermi constant and $N_{e}$ is the electron number density. For the antineutrino case, the interaction potential is

$$
\left(V_{\bar{\nu}_{e}}\right)_{C C}=-\left(V_{\nu_{e}}\right)_{C C}
$$

For the NC interaction the potential for all three types of neutrinos is the same since it is flavor independent. The mean interaction potential is described by $V_{N C}=-G_{F} N_{n} / \sqrt{2}$, where $N_{n}$ is the neutron number density. The contribution for $V_{N C}$ due to the electrons and the protons cancel each other out in a neutral environment. The total interaction 
potential $(C C+N C)$ is thus

$$
V_{e}=\sqrt{2} G_{F}\left(N_{e}-\frac{N_{n}}{2}\right), V_{\mu}=V_{\tau}=\sqrt{2} G_{F}\left(-\frac{N_{n}}{2}\right)
$$

The derivation of neutrino oscillation in matter is easier to derive in the flavor basis. For relativistic case for the two neutrino oscillations, we get

$$
i \frac{d}{d t}\left(\begin{array}{c}
\nu_{e} \\
\nu_{\mu}
\end{array}\right)=\left(\begin{array}{cc}
\left(p+\frac{m_{1}^{2}+m_{2}^{2}}{4 E}\right)-\frac{\Delta m^{2}}{4 E} \cos 2 \theta & \frac{\Delta m^{2}}{4 E} \sin 2 \theta \\
\frac{\Delta m^{2}}{4 E} \sin 2 \theta & \left(p+\frac{m_{1}^{2}+m_{2}^{2}}{4 E}\right)+\frac{\Delta m^{2}}{4 E} \cos 2 \theta
\end{array}\right)\left(\begin{array}{c}
\nu_{e} \\
\nu_{\mu}
\end{array}\right) .
$$

The terms in the parenthesis of the diagonal are the same and only change the common phase, and have no effect on oscillation probability. Thus the time evolution is described by

$$
i \frac{d}{d t}\left(\begin{array}{c}
\nu_{e} \\
\nu_{\mu}
\end{array}\right)=\left(\begin{array}{cc}
-\frac{\Delta m^{2}}{4 E} \cos 2 \theta & \frac{\Delta m^{2}}{4 E} \sin 2 \theta \\
\frac{\Delta m^{2}}{4 E} \sin 2 \theta & \frac{\Delta m^{2}}{4 E} \cos 2 \theta
\end{array}\right)\left(\begin{array}{c}
\nu_{e} \\
\nu_{\mu}
\end{array}\right)
$$

Let us now add the potential term. As seen from Equation 2.18 $N_{n}$ is common to both the potential terms and hence can be dropped, and we get (assuming no sterile interaction in matter),

$$
i \frac{d}{d t}\left(\begin{array}{c}
\nu_{e} \\
\nu_{\mu}
\end{array}\right)=\left(\begin{array}{cc}
-\frac{\Delta m^{2}}{4 E} \cos 2 \theta+\sqrt{2} G_{F} N_{e} & \frac{\Delta m^{2}}{4 E} \sin 2 \theta \\
\frac{\Delta m^{2}}{4 E} \sin 2 \theta & \frac{\Delta m^{2}}{4 E} \cos 2 \theta
\end{array}\right)\left(\begin{array}{c}
\nu_{e} \\
\nu_{\mu}
\end{array}\right) .
$$

In general, if the number density of the electrons is constant, we can diagonalize the 
effective Hamiltonian in matter:

$$
\begin{gathered}
\nu_{I}=\nu_{e} \cos \theta_{M}+\nu_{\mu} \sin \theta_{M}, \\
\nu_{I I}=-\nu_{e} \sin \theta_{M}+\nu_{\mu} \cos \theta_{M},
\end{gathered}
$$

where $\theta_{M}$ represents the mixing angle in matter and is described by

$$
\tan 2 \theta_{M}=\frac{\frac{\Delta m^{2}}{2 E} \sin 2 \theta}{\frac{\Delta m^{2}}{2 E} \cos 2 \theta-\sqrt{2} G_{F} N_{e}} .
$$

The eigenstates of $\nu_{1}$ and $\nu_{2}$ do not coincide with that of $\nu_{I}$ and $\nu_{I I}$ since $\theta_{M} \neq \theta$, the vacuum mixing angle. The difference in energies in matter is

$$
E_{I}-E_{I I}=\sqrt{\left(\frac{\Delta m^{2}}{2 E} \cos 2 \theta-\sqrt{2} G_{F} N_{e}\right)^{2}+\left(\frac{\Delta m^{2}}{2 E}\right)^{2} \sin ^{2} 2 \theta} .
$$

Finally, the neutrino oscillation probability in matter is

$$
P\left(\nu_{e} \rightarrow \nu_{\mu}\right)=\sin ^{2} 2 \theta_{M} \sin ^{2}\left(\frac{L\left(E_{I}-E_{I I}\right.}{2}\right)
$$

In the limiting case $N_{e} \rightarrow 0$, we get

$$
P\left(\nu_{e} \rightarrow \nu_{\mu}\right)=\sin 2 \theta \sin ^{2}\left(\frac{L}{2 E}\right)
$$

which is the familiar 2-neutrino vacuum oscillation equation. Thus the matter oscillation 
probability equation can be expressed as

$$
P\left(\nu_{e} \rightarrow \nu_{\mu}\right)=\sin 2 \theta_{M} \sin ^{2}\left(\frac{1.27 \Delta M^{2} L}{E}\right)
$$

where

$$
\Delta M^{2}=\Delta m^{2} \sqrt{\sin ^{2} 2 \theta+\left(\cos 2 \theta-\frac{\sqrt{2} G_{F} N_{e}}{\Delta m^{2} / 2 E}\right)^{2}},
$$

and we can see that if $N_{e} \rightarrow 0, \Delta M^{2} \rightarrow \Delta m^{2}$. The oscillation amplitude is described by

$$
\sin ^{2} 2 \theta_{M}=\frac{\left(\frac{\Delta m^{2}}{2 E}\right) \sin ^{2} 2 \theta}{\left(\frac{\Delta m^{2}}{2 E} \cos 2 \theta-\sqrt{2} G_{F} N_{e}\right)^{2}+\left(\frac{\Delta m^{2}}{2 E}\right) \sin ^{2} 2 \theta} .
$$

Maximal mixing occurs when $\Delta m^{2} / 2 E \cos 2 \theta=\sqrt{2} G_{F} N_{e},\left(\theta_{M}=45^{\circ}\right)$. This is known as the MSW effect, and it can occur independent from mixing angle in vacuum $\theta$. This implies that the probability for neutrino oscillation in matter can be large despite the smallness of the vacuum mixing angle! 


\section{Chapter 3}

\section{Previous Measurements}

\subsection{Introduction}

The neutrinos from atmospheric cosmic ray interactions provided the first compelling evidence for neutrino oscillations. Atmospheric neutrinos are produced from the decay of pions, kaons, and muons that are created in the upper atmosphere by the interaction of cosmic ray particles (mostly protons) with nucleons. The process is summarized as follows:

$$
\begin{aligned}
p+N & \rightarrow \pi^{ \pm} / K^{ \pm} \\
\pi^{ \pm} & \rightarrow \mu^{ \pm}+\nu_{\mu}\left(\bar{\nu}_{\mu}\right), \\
\mu^{ \pm} & \rightarrow e^{ \pm}+\nu_{e}\left(\bar{\nu}_{\mu}\right)+\nu_{\mu}\left(\bar{\nu}_{\mu}\right) .
\end{aligned}
$$


Thus we expect twice as many muon type neutrinos as electron type neutrinos. The double ratio $\mathrm{R}$, defined as the data ratio to the Monte Carlo ratio, is calculated for the electron and the muon type neutrinos as follows:

$$
R \equiv \frac{R_{D A T A}}{R_{M C}}=\frac{\left(N_{\mu} / N_{e}\right)_{D A T A}}{\left(N_{\mu} / N_{e}\right)_{M C}}
$$

where $N_{\mu}$ and $N_{e}$ are the number of muon type neutrino events and the electron type neutrino events respectively. A significant deviation from unity in the measured ratio would indicate an anomaly. Earliest measurement was done by the IMB experiment [11], and later confirmed by the Kamiokande experiment [13]. The double ratios measured by the experiments were $R=0.54 \pm 0.05$ (stat) \pm 0.11 (syst) by the IMB experiment [12], and $R=0.60_{-0.06}^{+0.07}($ stat $) \pm 0.05$ (syst) by the Kamiokande experiment [13]. These were among the first indications of the atmospheric neutrino anomaly.

Other measurements done by the NUSEX [14] [15] experiment and the Frejus [16] experiment found no real deviation from unity in the double ratio measurement. The Soudan 2 Experiment [21] resolved this discrepancy and confirmed the IMB and Kamiokande results. The double ratio measured by the Soudan 2 collaboration was $R=0.72 \pm$ $0.19(\text { stat })_{-0.07}^{+0.05}($ syst $)[80$. The NUSEX and Frejus results are believed to be due to fluctuations of their data. The atmospheric neutrino anomaly is currently believed to be due to the flavor oscillation between the three types of neutrinos.

One way to observe neutrino oscillation is by measuring the zenith angle distribution 
of the neutrinos. For neutrino detectors close to the surface of the earth the zenith angle corresponds to a wide range of neutrino distances that the neutrinos traverse $(L \sim$ $20-13,000 \mathrm{~km}$ ). If neutrinos do oscillate in these distances, then a deficit in the measured $\nu_{\mu}$ flux will occur at distances $\sim L$ that corresponds to a high probability of oscillation. The Super-Kamiokande experiment has made compelling measurements which indicate that neutrino oscillation is a real phenomenon.

\subsection{The Super-Kamiokande Experiment}

The Super-Kamiokande ("Super-K") [20] is $50 \mathrm{k}$-ton water Cerenkov detector located in Mozumi mine in Japan and has a mean over burden of 2700 meters of water equivalent. The inner detector is filled with pure water and has a cylindrical volume with a radius of 16.9 meters and a height of 36.2 meters. The signal from the neutrino interactions is received by 11,146 Hamamatsu R3600 photomultiplier tubes (PMT). The PMTs have a timing resolution of 2.5 ns. The large fiducial volume provides a big sample of multi-GeV charged current (CC) events. The outer detector containing a layer of water completely surrounds the inner detector, and is used as a shield from radioactive material from the rock that surrounds it. The outer detector data are used to identify incoming cosmic rays and outgoing muons from neutrino interaction from the detector.

The atmospheric events are divided into three categories: Fully Contained (FC), Partially Contained (PC), and neutrino induced Up-Going Muons (UPMU). A recent analysis shows that the multi-GeV $\nu_{\mu}$ distribution is approximately half the expected value 
at large zenith angles [17. Whereas the $\nu_{e}$ distributions show good agreement with the expectation. This was inferred as atmospheric neutrinos going through $\nu_{\mu} \rightarrow \nu_{\tau}$ oscillations at maximal mixing. The $90 \%$ confidence limit were set at $1.5 \times 10^{-3} \mathrm{eV}^{2}<$ $\Delta m_{23}^{2}<3.4 \times 10^{-3} \mathrm{eV}^{2}, \sin ^{2} 2 \theta_{23}>0.92$, with the best fit oscillation parameters at $\Delta m_{23}^{2}=2.1 \times 10^{-3} \mathrm{eV}^{2}, \sin ^{2} 2 \theta_{23}=1.0[17]$.

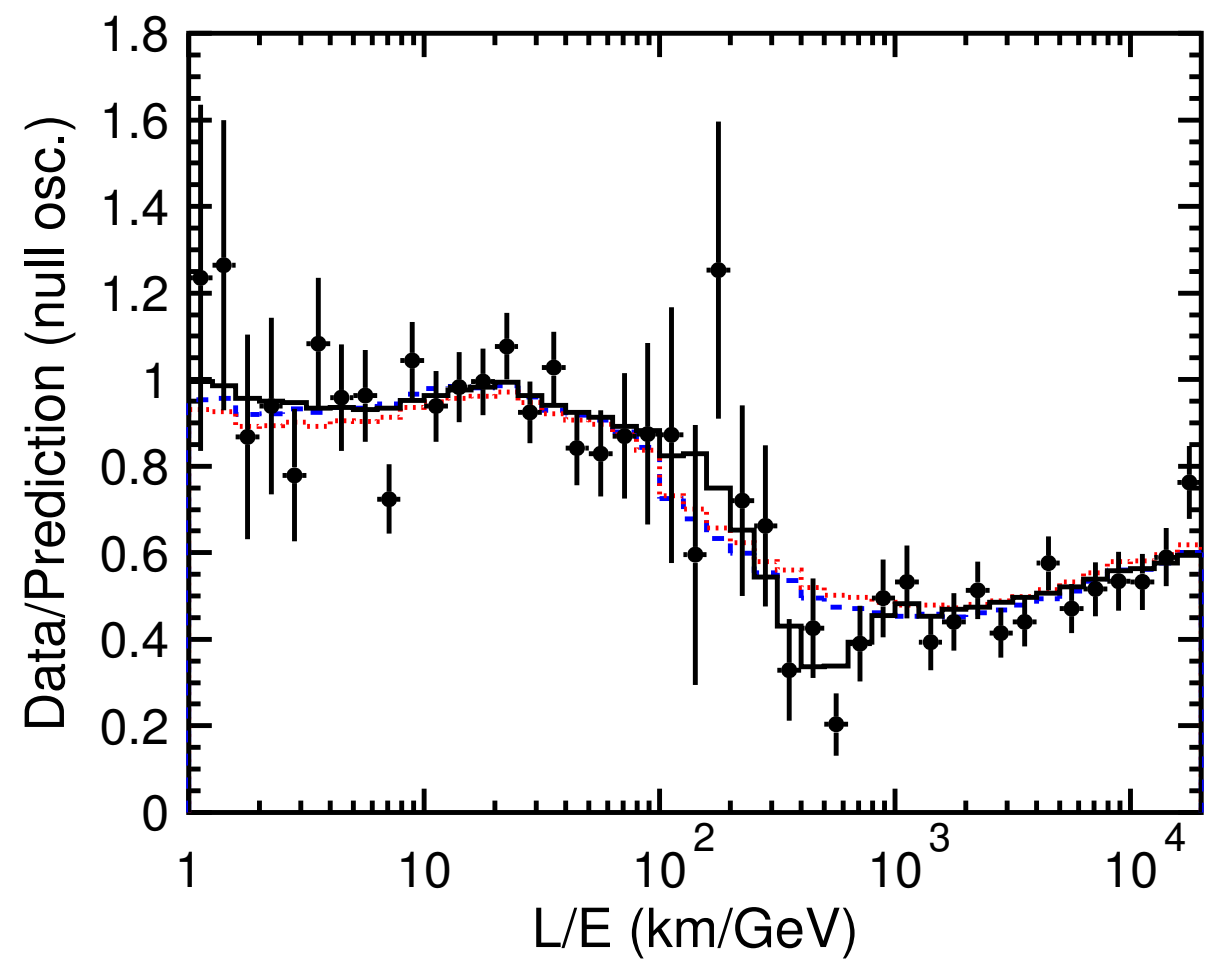

Figure 3.1: $L / E$ analysis for Super-K. The ratio of the measured $L / E$ distribution and the expectation in the absence of oscillation is included. The Solid line represents the best fit neutrino oscillation; dashed line represents neutrino decay; dotted line represents neutrino de-coherence [18].

Another measurement by the Super-K Collaboration [18] presented an $L / E$ oscillation analysis. The $L / E$ resolution was calculated as a function of log of the zenith angle and 
the neutrino energy. Monte Carlo studies with $L / E$ resolution of $<70 \%$ in data was used for oscillation analysis. The region with $L / E$ resolution $>70 \%$ are horizontal events. A dip has been observed at $L / E \simeq 500 \mathrm{~km} / \mathrm{GeV}$ which is consistent with the oscillatory periodicity of probability function. This is illustrated in Figure in 3.1 which shows the ratio of the data to the unoscillated $\mathrm{MC}$ as a function of the reconstructed $L / E$ values. The solid line represents the best fit expectation for the two flavor oscillation. The $90 \%$ confidence limits were set at $1.9 \times 10^{-3} \mathrm{eV}^{2}<\Delta m_{23}^{2}<3.0 \times 10^{-3} \mathrm{eV}^{2}$, and $\sin ^{2} 2 \theta_{23}>0.90$. The best fit parameter is found at $\Delta m_{23}^{2}=2.4 \times 10^{-3} \mathrm{eV}^{2}$ at maximal mixing [18]. This is consistent with the results from the zenith angle analysis by the collaboration. This measurement also rejects the alternative theories like neutrino decay [26] and neutrino de-coherence [27] to neutrino oscillation at 3.4 standard deviations since neither theories predict a dip in the $L / E$ distribution.

A separate Super Kamiokande analysis has been performed and published by looking at the neutrino induced muons in observed in the detector [19]. In this analysis, the upward going neutrino induced muons were separated into two categories. The neutrino induced muons that stopped inside the fiducial volume of the detector with a track length greater than seven meters long were called "upward stopping muons". The MC studies showed that the typical energy for the parent neutrinos of these stopping muons are $10 \mathrm{GeV}$. The neutrino induced muons that cross the entire detector were called "upward through-going muons," with typical parent neutrino energy around $100 \mathrm{GeV}$.

The oscillation effects were measured by taking the ratio of the stopping muon flux 


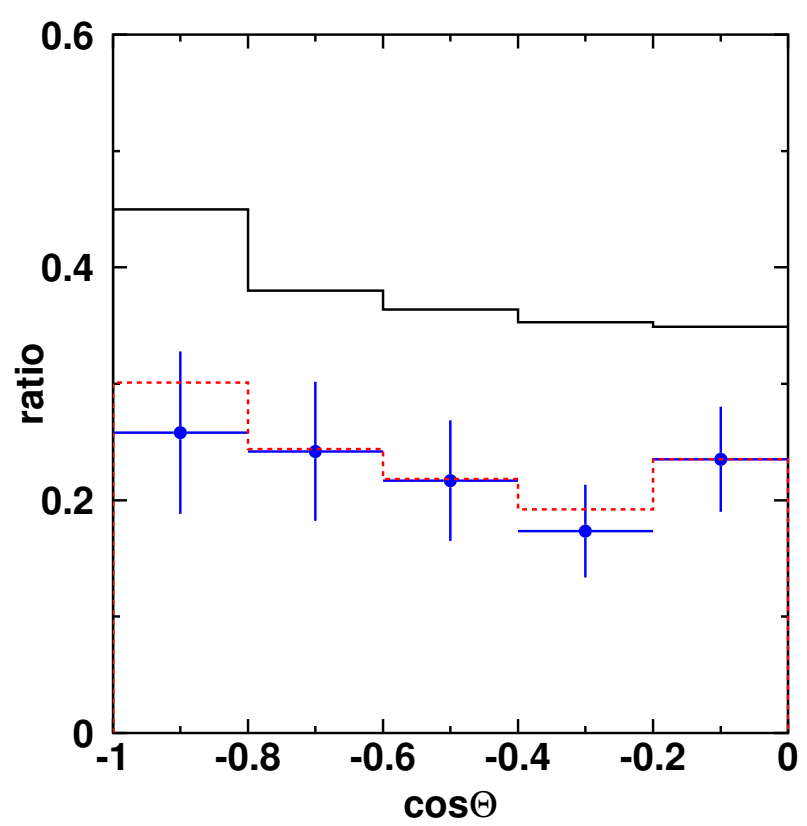

Figure 3.2: The upward stopping/through-going neutrino induced muon ratio as a function of cosine of the zenith angle. The solid histogram represents the unoscillated MC expectation. The dashed histogram represents the oscillated MC expectation.

to the through-going muon flux, $R=\Phi^{\text {stop }} / \Phi^{\text {thru }}$. Figure 3.2 shows the measured $R$ as a function of cosine of the zenith angle of the neutrino induced muons. The oscillated and the unoscillated MC expectations are shown by the dashed and the solid histogram respectively. There is a clear deficit in the muon neutrino flux indicating neutrino oscillations. The combined zenith angle and the upward stopping/through going ratio oscillation fit analysis was also done. Figure 3.3 shows the result of oscillation fit. The $68 \%$ (dotted line), the $90 \%$ (thick solid), and the $99 \%$ (dashed line) contours are compatible with the contained analysis [18]. 


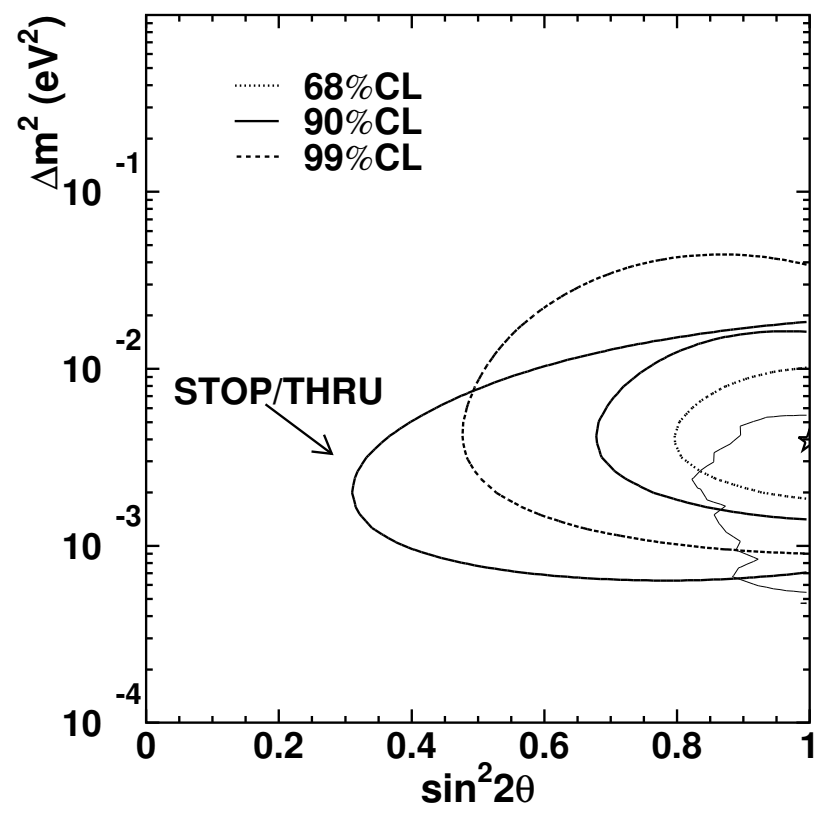

Figure 3.3: The allowed regions calculated from the combined zenith angle and stopping/though-going ratio oscillation fit. The best fit point is indicated by the star at $\left(\Delta m^{2}, \sin ^{2} 2 \theta\right)=\left(3.9 \times 10^{-3} \mathrm{eV}^{2}, 1.0\right)[19$. The Super-K contained analysis result (at $90 \%$ C. L.) is shown by the thin solid line.

\subsection{The Soudan 2 experiment}

The Soudan 2 experiment [22], located at the same depth as the MINOS Far Detector, was a time projection, iron calorimeter with a modular design. The experiment started taking data from April 1989 and ceased to operate in June 2001. The detector had a honeycomb geometry. The tracking elements of the detector were $1 \mathrm{~m}$ long and $1.5 \mathrm{~cm}$ diameter hytrel plastic drift tubes filled with an argon-CO2 gas mixture. The tubes were encased in honeycomb matrix with $1.6 \mathrm{~mm}$ thick corrugated steel plates. The deposited electrons 
from the passage of charged particles drifted under the influence of electric field to the end of the tubes where the signal was magnified by the vertical anode wires. The induced charge was read out by horizontal strips and the drift time for the electrons provided the third coordinate. The ionization was measured by the anode pulse height. The detector produced three dimensional track hits with approximately $1 \mathrm{~cm}^{3}$ spatial resolution. The detector was also constructed with a Veto Shield that surrounded it completely. The shield hits were analyzed to veto the cosmic ray muon events and the background events from the surrounding rock.
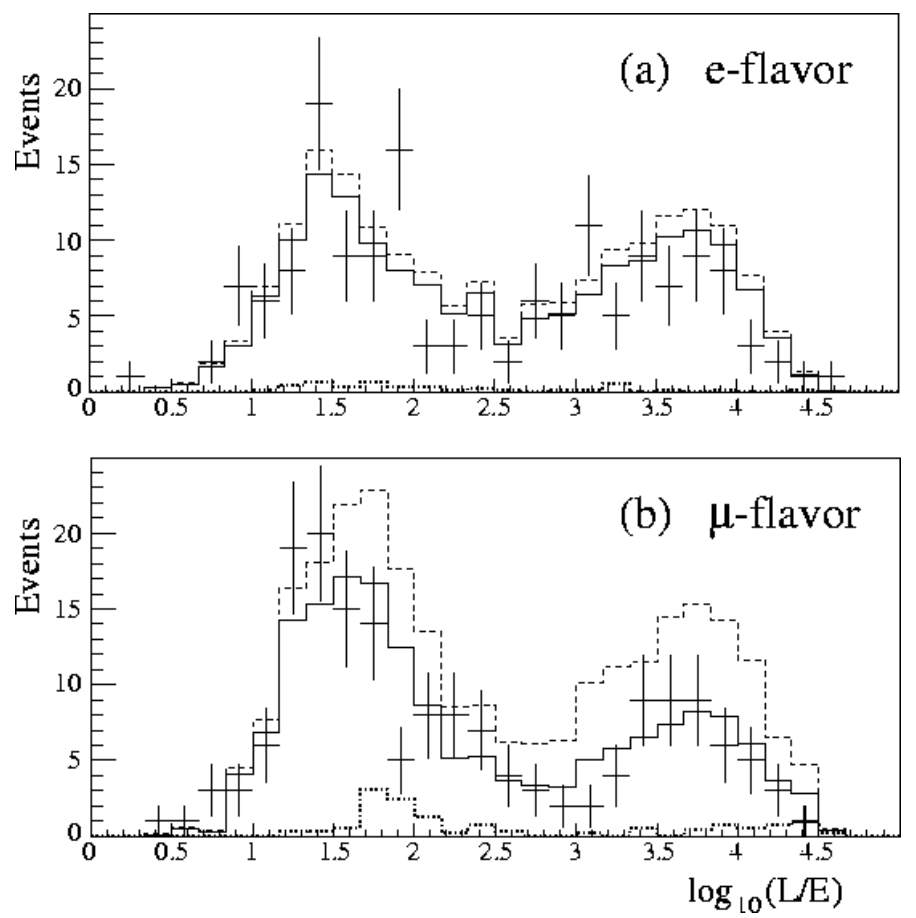

Figure 3.4: The high resolution $L / E$ distribution e-like and the $\mu$-like events. The data are shown with the error bars. The dashed histograms are the no oscillation expectation. The solid histograms are the oscillated prediction with the best fit parameters. The dotted histograms represent the background. 
The collaboration published an $L / E$ oscillation measurement with 5.90 kton-years of data 24] that consisted of fully contained and partially contained events. A sample of "high resolution" events were extracted by placing several energy cuts on the track events and the shower events. These events have more accurate neutrino pointing and energy resolution than the low resolution events. The track events were called the " $\mu$-flavor" events, while the shower events were called the "e-flavor" events.

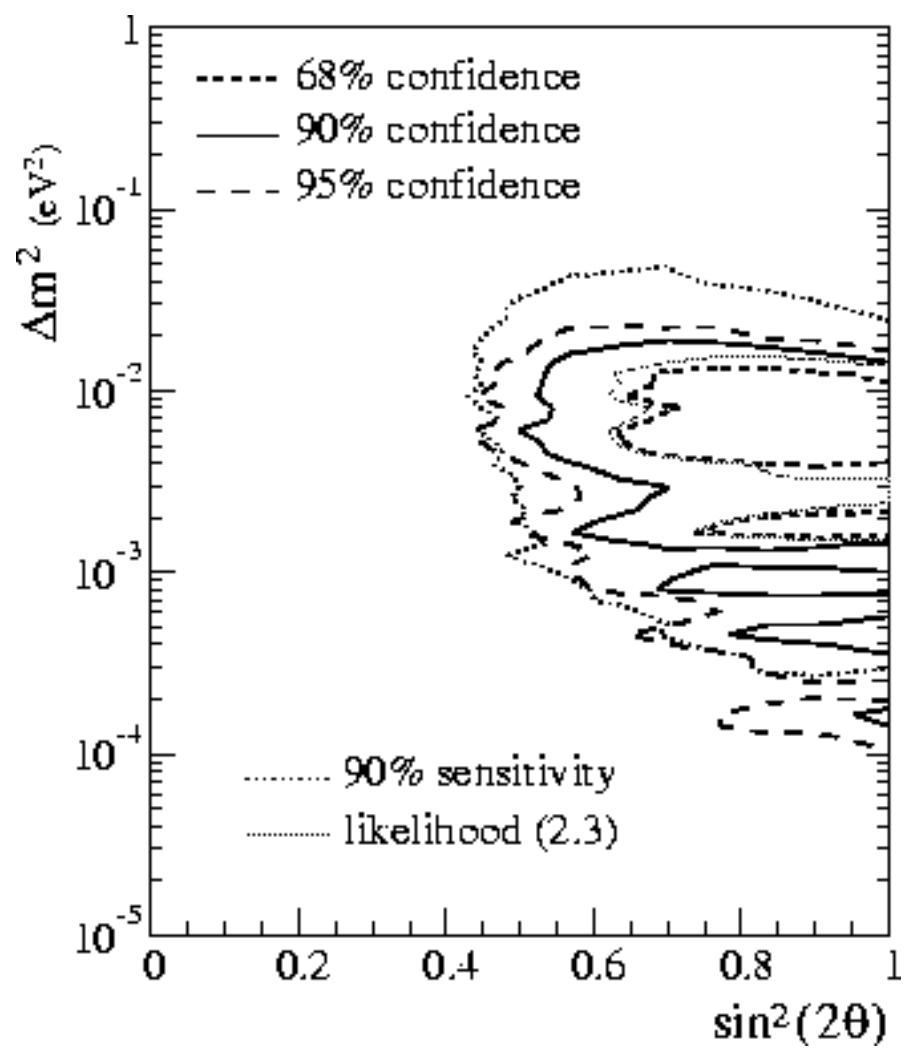

Figure 3.5: The confidence level contours generated by the Soudan 2 analysis using Feldman Cousins approach [25]. Also shown are the contours for the $90 \%$ sensitivity (dotted line), and the contour defined by a data likelihood rise of 2.3 . 
With the events identified as muon-like or electron-like events, the flavor double ratio was measured as

$$
R=0.69 \pm 0.10(\text { stat }) \pm 0.06(\text { syst }) .
$$

The oscillation analysis was done with the measured $L / E$ values assuming the dominant two flavor $\nu_{\mu} \rightarrow \nu_{\tau}$ oscillations. Figure 3.4 shows the measured and the expected $L / E$ distribution for the muons-like and the electron-like events. There is a clear depletion in the $\mu$-like events from the no oscillation expectation for higher values of $L / E$. Figure 3.5 shows the $68 \%, 90 \%$, and the $95 \%$ contour levels generated as a result of the oscillation fit analysis. The best fit oscillation parameter values were $\Delta m^{2}=0.0052 \mathrm{eV}^{2}$ and $\sin ^{2} 2 \theta=$ 0.97. The results are compatible with the best fit Super Kamiokande oscillation parameters [18].

The Super-K, Soudan 2, and other experiments have seen evidence of neutrino oscillations. This dissertation uses neutrino induced muons to measure the parameter values of $\Delta m^{2}$ and $\sin ^{2} 2 \theta$. Although the MINOS Far Detector was designed for a long baseline beam experiment, atmospheric neutrino oscillation analysis can be performed nonetheless. In order to select the neutrino induced muons, the workings of the detector must be understood. This is the topic of discussion for the following chapter. 


\section{Chapter 4}

\section{The MINOS Far Detector}

\subsection{Introduction}

Located at a depth of 2100 meter water equivalent (m. w. e.) in Soudan Underground Mine in Soudan, Minnesota, USA, the MINOS Far Detector is a 5.4 KTon iron calorimeter. The primary design goal for this device is to efficiently detect, distinguish and measure the energy of muon-type neutrinos through their charged current interaction in iron. The Far Detector is characterized by both its high density and high Z. This design both maximizes the detector efficiency and differentiates between muons from other particles by the ability of muons to penetrate long distances in iron without significant interaction. The MINOS Far Detector design is clearly optimized for the observation of beam neutrinos from Fermilab, with, for example, detector planes oriented perpendicular to the beam axis. However, except for nearly vertical upward going muons, the MINOS Far Detector 
also has good efficiency for atmospheric neutrino events.

This chapter gives the general physical overview of the MINOS Far Detector with an emphasis of features particularly relevant to the design goals. It describes in somewhat details the scintillator system, followed by the photodetectors, electronics and the data acquisition system. A brief description of physics event information is also given. The MINOS Far Detector is unique with it's ability to distinguish between the positively and negatively charged leptons by curvature in the detector's magnetic field. Details of the detector magnetics are also described, preceding a discussion of the calibration of the detector.

\subsection{General Overview}

The MINOS far detector is comprised of 484 planes of scintillator sandwiched between 485 planes of steel. Each plane is octagonal in shape and has a diameter of $8 \mathrm{~m}$. The detector is split into two supermodules. The southern module (closest to Fermilab) has 248 planes. The northern spuermodule has 243 planes. Each supermodule is fitted with $15 \mathrm{kA}$-turn coil that runs through a hole in the center of each plane and returns along the floor under the detector. The current in this coil generates a toroidal magnetic field with field lines lying within the iron planes and circling the central coil hole. The average magnetic field is $\sim 1.2 \mathrm{~T}$. This magnetic field allows both charge identification and momentum measurement of muons from charged current muon neutrino interactions.

Each detector plane consists of a layer of $2.54 \mathrm{~cm}$ thick steel absorber and a $1 \mathrm{~cm}$ thick 


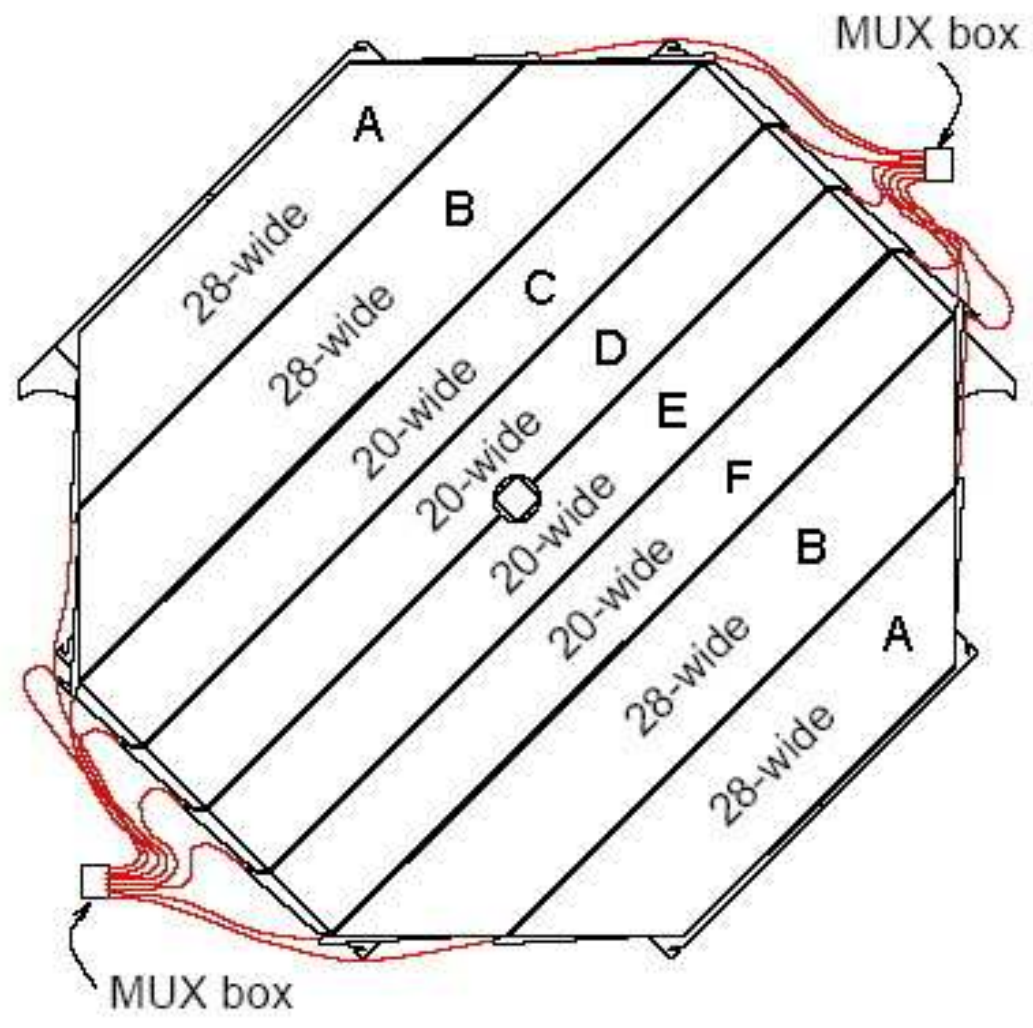

Figure 4.1: The overall arrangement of a scintillator plane showing the module positions and the multiplexed wavelength-shifting fiber optical readout.

plastic scintillator strips as an active detector. The spacing between plane centers is 9 $\mathrm{cm}$, leaving an air gap of $2.54 \mathrm{~cm}$ between planes. The arrangement of the scintillator and steel planes is shown in Figure 4.1. There are 8 scintillator modules (labeled) comprising each plane. Each module is encased in a thin aluminum shell to protect the scintillator and keep out external light. There are two basic module types. One type consists of 20 extruded polystyrene strips (known as CalTech modules); the other type has 28 similar 
strips (known as Minnesota modules). The Caltech modules, labeled C, D, E, F, cover the inner portion of the plane while the Minnesota modules, labeled A and B, cover the outer edge. Caltech modules have scintillator strips that are $\sim 8 \mathrm{~m}$ in length, although the strips located near the coil hole are shorter. Minnesota modules contain 28 strips that vary from $\sim 4-8 \mathrm{~m}$ in length. There are 192 strips per detector plane.

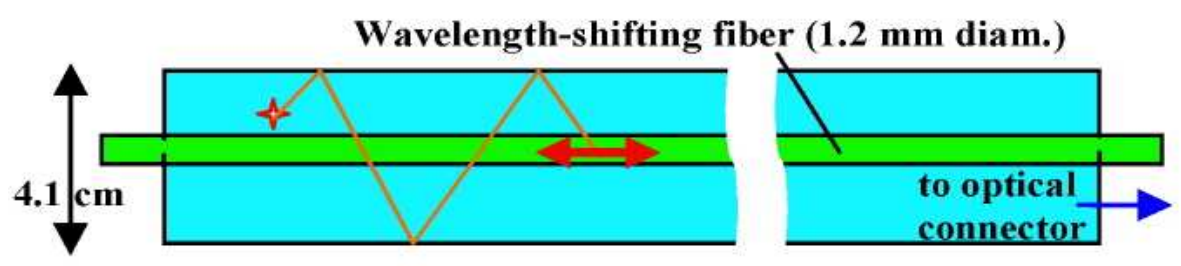

$1.0 \mathrm{~cm} \times 4.1 \mathrm{~cm}$ extruded polystyrene scintilaltor

Figure 4.2: The details of a scintillator strip showing the location of the fiber optical readout.

The $1 \mathrm{~cm}$ thick extruded polystyrene scintillators are $4.1 \mathrm{~cm}$ wide as shown in Figure 4.2. Each strip is co-extruded with an external layer of a $\mathrm{TiO}_{2}$ reflective coating and a central $1.3 \mathrm{~mm}$ diameter groove on the flat side for a $1.2 \mathrm{~mm}$ diameter wavelength-shifting fiber (WLS fiber). These WLS fibers are glued to the groove of each scintillator. Light produced within each scintillator is collected by the fiber glued to that strip and carried to the clear readout fibers at both ends of the detector. These clear fibers carry the light to multiplexer (MUX) boxes, which combine light from 8 well-separated scintillator strips. The multiplexing arrangement differs at the two strip ends, to permit software 
demultiplexing prior to event reconstruction. The multiplexed light is then routed to $16-$ pixel Hamamatsu R590-00-M16 photomultiplier tubes (PMTs) housed in the MUX box. Figure 4.3 shows the cross-section of the detector including the Veto Shield.

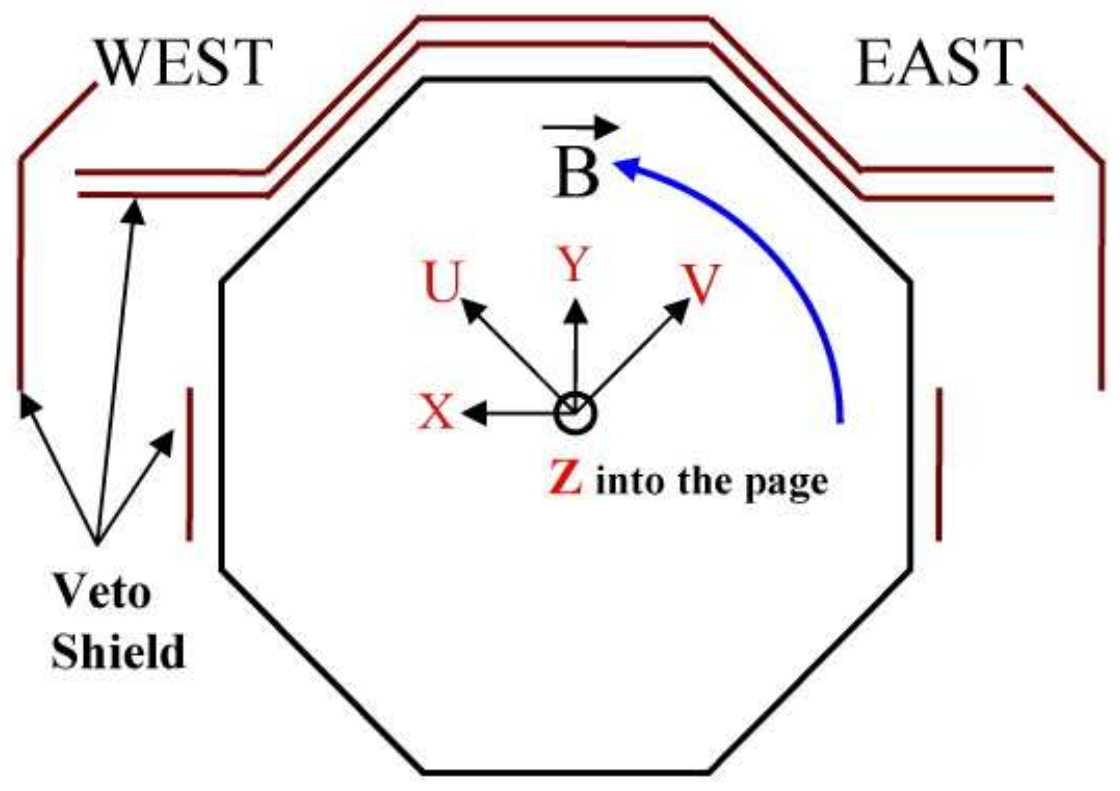

Figure 4.3: Overall schematic of the detector showing the coordinate systems used for event reconstruction.

\subsection{Scintillator}

The MINOS Far Detector is similar in size to particle detectors at collider experiments, but it differs from those devices because it consists of one rather than many primary detector systems. Compared to other particle detection techniques, the advantages of 
the extruded polystyrene scintillator used in MINOS are relative simplicity, stability and straightforward operation. The scintillator also facilitates nanosecond timing resolution, which is particularly important for this research in separating upward going from downward going muons by a factor of at least $10^{5}$. On the other hand, cost limitations result in a detector with limited spatial resolution and coarse sampling (with resulting poor energy resolution) for both hadronic and electromagnetic showers.

As described above, the extruded polystyrene scintillator strips used in the MINOS Far Detector have an external width of $4.1 \mathrm{~cm}$, a thickness of $1 \mathrm{~cm}$ and a length of up to $8 \mathrm{~m}$, all dimensions including the co-extruded $\mathrm{TiO}_{2}$ reflective outer coating. The active fluors in the polystyrene are a mixture of PPO and POPOP. The components of the scintillator system are known to slowly age, with the scintillator light output slowly dropping and the photomultiplier gain slowly increasing over time. A light injection calibration system is used to measure scintillator and PMT aging and other possible time-dependent variations on a strip-by-strip and pixel-by-pixel basis. The data from the light injection system is accumulated in a database and used to adjust the raw data during event reconstruction.

In order to determine the absolute energy calibration and the hadronic and electromagnetic energy resolutions of the MINOS Far Detector, a small detector was built using the same design. This Calibration Detector (Caldet) was then exposed to a test beam at the CERN PS. Using the Caldet detector, the energy resolutions of the MINOS design were measured as $\sim 23 \% / \sqrt{E}$ for electromagnetic showers and $\sim 53 \% / \sqrt{E}$ for hadronic showers. These energy resolutions are not directly relevant to the research described here, 
which actually measures only charged muon tracks.

\subsubsection{Fiber}

The readout of long plastic scintillator strips is difficult because of self-absorption within the scintillator. One technique for addressing this problem is the use of wavelengthshifting fiber. The wavelength shifter absorbs ultraviolet light, which comprises much of the scintillator light output and which is not well transmitted by even non-scintillator plastic, and re-emits the light as green $(\sim 500 \mathrm{~nm}$, where the fiber has a relatively long, $5 \mathrm{~m}$ attenuation length. At the scintillator strip ends, the WLS fiber is joined to clear fiber with an even longer, $12 \mathrm{~m}$ attenuation length. The WLS fiber is coated with two layers, to maximize transmission. The inner coating layer has an index of refraction of 1.55 compared to an index of refraction for the fiber core of $n=1.49$. The refraction index for the outer coating layer is 1.59. The WLS fiber used in MINOS is manufactured in Japan by Kuraray.

The clear optical fiber is routed in multi-fiber plastic casings along the edge of the detector steel planes to multiplexing (MUX) boxes. Within each MUX box, an array of fibers directs light from 8 well-separated scintillator strips to a single pixel in an optical disk or "cookie," which is then mated to the face of the multi-anode photomultiplier tube (PMT). The fibers are aligned to the appropriate PMT pixel with a resolution of $125 \mu \mathrm{m}$. The two ends of each scintillator strip are multiplexed differently to facilitate software de-multiplexing. The demultiplexing algorithms also use pulse height, pulse timing and 
multiplane tracking in order to resolve individual scintillator "hits".

\subsection{Photomultiplier Tubes (PMT)}

Three Hamamatsu R5900-00-M16 photomultiplier tubes are located in each MUX box. The MUX boxes are rack mounted along the upper and lower east and upper and lower west sides of the detector. Each photomultiplier tube has a bialkalai cathode coated onto a $1 \mathrm{~mm}$ thick borosilicate glass window. These PMTs are sensitive over the range of 300 to $650 \mathrm{~nm}$, with maximum sensitivity at $520 \mathrm{~nm}$ and a quantum efficiency of

13 percent at $420 \mathrm{~nm}$. The pixel size is $4 \mathrm{~mm}$ square. The average gain is $10^{6}$ at a working voltage of 800 to 1000 volts. The PMT timing jitter is $<2 \mathrm{~ns}$ for more than 10

photons. As a result, the overall timing resolution for small signals is dominated by timing jitter in the wavelength shifting fluor. The PMT gain is linear up to 20 photoelectrons. The dark current is less than $1 / 3$ photoelectron. Overall, the gain variations are well understood. The multichannel PMT is subject to cross-talk among pixels, but the amount of crosstalk is measured pixel-by-pixel and a software correction for cross-talk is applied during reconstruction.

\subsection{Electronics}

The MINOS Far Detector electronics seeks to acquire maximum information about the output of each PMT channel by digitizing each channel's waveform as a function of time 
and organizing and transmitting this information for later analysis. The electronics design takes advantage of the low rate underground environment in which the apparent signal rate is dominated by electronic noise of $3-6 \mathrm{kHz}$ per PMT. In this environment, a high speed digitizer can service many channels while maintaining a very low deadtime.

The readout electronics is based on a front end ASIC VA32-HDR11 (called a VA chip), developed in cooperation with the Norwegian company IDEA ASA. These chips provide a charge sensitive preamplifier, a shaper and a sample and hold for each PMT anode channel. The Outputs on all channels are processed in parallel. The VA chip also includes an analog output multiplexer, so that each of the 16 anode channels from a PMT can be addressed. Three VA chips are mounted on a VA Front End Board (VFB), one of which is attached to each MUX box. The VFBs are fully controlled by a VA Readout Controller (VARC) module. The VARC modules, each of which provides the readout and calibration control for 12 VFB's, are located at the mid-plane of the detector and are connected by twisted pair cables to VFB's above or below them. Each VARC houses 6 VA Mezzanine Modules (VMM). Each VMM performs analog to digital conversion for two VFB's, that is, 6 PMT's.

In addition to the multi-pixel anode outputs, PMT last dynode outputs are processed by the electronics to implement a readout trigger. The dynode signal is discriminated and then time-stamped with a $640 \mathrm{MHz}$ TDC. This trigger provides a 500 ns "hold" signal to the VA chip prior to digitization. There is a deadtime of $5 \mu s$ per dynode trigger. The system has been tuned so that 1 ADC count corresponds to $2 \mathrm{fC}$. The electronic noise in 
the fully installed system is typically around 2.5 ADC counts or 5 fC. The noise changes by less than 1 ADC count over 24 hours. The entire detector readout is synchronized by a $40 \mathrm{MHz}$ optical timing distribution signal slaved to a GPS clock.

The ACD outputs are stored in a local FIFO for further processing under control of the VARCs. This processing includes pedestal and common noise subtraction and sparsification. The data are then written into a VME memory and read out by the Data Acquisition system (DAQ).

\subsection{Data Acquisition System (DAQ)}

The principal function of the Data Acquisition System (DAQ) is to continuously read out the front end electronics in an untriggered, deadtime free mode and to transfer that data to trigger PC's for selection of interesting events and to perform monitoring and calibration tasks. Sixteen VME mounted computers called Readout Processors (ROP), one in each data crate, assemble consecutive time blocks into memory. Each time block, nominally one second in length, carries a header containing a full, self-description of the data in the block. Datablocks from monitoring and calibration tasks are appended to the time-frames as necessary. ROP's are PowerPC's running VxWorks that includes all the readout-specific software for the front-end electronics systems. The ROP's are daisy-chained together into branches and each branch is connected to a Branch Readout Processor (BRP). The BRP's control the transfer of data from the ROP's to the trigger PC's. One BRP acts as the master, coordinates the transfer of data and selects an available Trigger Processor (TP) 
for each data set. The TP then examines the time frame to determine whether it contains data of interest. The TP divides the data stream into segments separated by at least 150 ns with no hits. Each of these segments or candidate events are evaluated using flexible software algorithms.

Candidate events passing an algorithm are written to an output data stream. The data streams are written in parallel, that is, a candidate event can satisfy more than one trigger algorithm and therefore be written to more than one data stream. While several data streams are associated with "spill" triggers time-coincident with beam from Fermilab, the most important data stream for the analysis reported here is the "Plane Trigger." This trigger requires $\mathrm{M}$ out of $\mathrm{N}$ consecutive planes to contain at least one hit. Although the values of $\mathrm{M}$ and $\mathrm{N}$ can be changed by software, the $\mathrm{M}$ and $\mathrm{N}$ values that have been used since the detector began operation are $\mathrm{M}=4$ and $\mathrm{N}=5$. Because a time frame can contain more than one interaction or event, the time frames selected by the Trigger Processors are called "snarls." Snarls written to an output buffer include $30 \mu$ s of detector activity prior to snarl. This "pre-trigger window" is used to provide sufficient data to identify "dead" channels. Snarls are written and archived as ROOT files for off-line reconstruction analysis.

\subsection{Physics Event Information}

The lowest level of data in each snarl archived for off-line reconstruction are called "candidate digits" or "cand-digits." Each cand-digit includes an electronics channel number, the 
digitized pulse height and time-stamp indicating when the cand-digit was observed. The channel number identifies the specific photomultiplier pixel associated with the cand-digit. Because of optical multiplexing, each pixel is associated with 8 physical scintillator strips. The multiplexing pattern is different on the east and west sides of the detector, so that if a single scintillator strip in a plane produces detectable light at both ends and no other strip in the plane fires, that strip is uniquely identifiable by comparing the east and west cand-digits for that plane. If more than one strip is hit, de-multiplexing may or may not be unique. De-multiplexing algorithms were developed to address this situation by using information from more than one plane [32]. For most event topologies, the de-multiplexing algorithms are highly successful in determining an unique assignment for each cand-digit to a physical scintillator strip. The de-multiplexed "hits" are then passed to track-finding algorithms.

A Hough Transform forms the core of the track-finding algorithms. This transform takes hits from physical space and maps them into a space where parallel lines cluster. A large cluster of hits in the Hough Transform space defines a linear trajectory or "highway" through a set of hits. Of course, the more planes that form a track, the higher the efficiency for finding the track. However, experience with the MINOS Far Detector indicates that track-finding efficiency plateaus for 7 or more planes [33]. Once tracks are found, the analysis software uses a Kalman Filter [75] algorithm to recursively decide which hits to include into the final fitted track. The track fitter uses a magnetic field map (described below $)$ in the fitting process. For low momentum tracks $(\leq 20 \mathrm{GeV} / \mathrm{c})$, the curvature in the 
toroidal magnetic field uniquely defines both the charge and momentum of the track. For higher momentum tracks (up to $\sim 100 \mathrm{GeV} / \mathrm{c}$ ), the curvature provides some information about charge and momentum, but both measurements have some uncertainty.

\subsection{Magnetics}

The MINOS Far Detector is the first large underground detector with a magnetic field. As a result, MINOS is able to make new measurements of the charge and energy spectrum of deep underground cosmic ray muons. The toroidal magnetic field in the Far Detector is produced by a $15 \mathrm{kA}$ coil (Figure 4.3), which runs through a center hole in the Detector and then returns underneath the Detector. The Detector is divided into two "Supermodules" and each Supermodule has its own independent coil. The magnetic field in the iron produced by this current varies from $\sim 1.7 \mathrm{~T}$ near the central coil hole to $\sim 1.2 \mathrm{~T}$ near the outer edges of the Detector. The field also varies slightly from plane to plane depending on the exact chemical composition, and rolling and heating history of the iron. A field map (B field as a function of position) has been determined by an ANSYS Finite Element Analysis calculation [34] and verified by actual measurements on the Detector. Because of the constant evolution of the software, slightly different field maps have been used for the Monte Carlo simulation and the data analysis, but the map changes do not, in general, produce physically different results. Both the Monte Carlo simulation and the data analysis programs use the field map to determine the relationship between particle charge and momentum and track curvature. 


\subsection{Calibration}

A considerable effort has been devoted to determining calibration constants to correct for non-uniformities in the MINOS Far Detector. These inhomogeneities in pulse height are particularly important for shower reconstruction in beam-related events. They are generally not very important for track analysis that is necessary for the studies described here. As long as a "hit" produces a pulse-height above some minimum threshold, it will contribute correctly to a track analysis, regardless of its exact pulse height. The calibration that is important for this analysis is timing, which is used to distinguish upward-going and near horizontal muons from the much larger number of downward going muons. For this reason, a brief description of the calibration procedure is given here.

Inhomogeneities in the Detector exist in both the light output and the electronic gain. The light output can be non-uniform because of the intrinsic properties of each scintillator strip, its Wavelength-Shifting Fiber and the clear fiber that carries the light to the photomultiplier tube (PMT). The electronic gain can vary because of the properties of the PMT's and the amplification and digitization circuitry that processes the PMT outputs. Light variations generally affect one strip. Electronic variations generally affect multiple strips, in some cases pixel-by-pixel and in other cases for the PMT as a whole.

The calibration process performs both pulse-height and timing calibration. It includes light injection, charge injection and cosmic ray calibration. Light Injection (LI) measures and corrects for non-linearities, gain drifts over time and also measures the observed pulse height associated with the single photoelectron peak for each PMT. The system uses blue 
LED's to produce the injected light and PIN to monitor the injected light level separately from the normal detector electronics signal-analyzing path. Charge injection calibration is built into the front-end chip, which can place a known charge into each electronics channel. Reading out the ADC associated with that channel provides a $1 \%$ calibration of the pulse height electronics [36]. Finally, the reconstruction of minimum-ionizing cosmic ray muons provides an overall check on the detector. Of course, the pulse height with such a muon depends on the muon path length through the scintillator, which depends on the angle between the track and the detector $\mathrm{z}$ axis. However, at the depth of the MINOS Far Detector, there are a sufficient number of high energy muon tracks to provide useful information about pulse height, position and timing of every detector strip. The timing resolution, which is important for the analysis reported here, is $2.4 \mathrm{~ns}$ [32]. The width of the $1 / \beta$ distribution is an indicator of the quality of the timing corrections. 


\section{Chapter 5}

\section{Cosmic Ray Muons and}

\section{Atmospheric Neutrinos}

\subsection{Introduction}

In order to verify neutrino oscillations in the MINOS Far Detector the flux and the simulation of the cosmic ray muons and the atmospheric neutrinos must be studied. In this chapter an account for the cosmic ray flux and the simulation of the cosmic ray muons is given. The predicted distributions are compared to the collected data to verify the detector response. A description of the flux and the simulation of the atmospheric neutrinos is included with the uncertainty in the predicted flux. Finally, the cross section for the atmospheric neutrinos is discussed. 


\subsection{Cosmic Ray Muons}

The cosmic ray muons contribute to the largest fraction of events recorded in the MINOS Far Detector. The cosmic ray analysis is important in several ways. First, the cosmic ray analysis provides the means understanding the detector well. For example, the acceptance of the detector can be determined by using the cosmic ray muons. The acceptance of the detector has been measured to be $7.6 \times 10^{6} \mathrm{~cm}^{2}$ sr by Brian Rebel [32]. The detector timing can also be calibrated using the cosmic ray muons. As discussed in Chapter 4 the strip timing resolution for the Far Detector is 2.3 ns. In this section, the flux of these cosmic ray muons is discussed, along with the simulation of the underground cosmic ray muons. The data distributions the is compared to that of the the Monte Carlo (MC).

\subsubsection{Cosmic Ray Flux}

The primary cosmic ray particles constantly bombard the Earth at a rate of $\sim 1000$ $m^{-2} s^{-1}$. The composition of these particles are $95 \%$ protons, $4 \%$ alpha particles, and $1 \%$ heavier nuclei 37] 38]. The primary cosmic rays have a vast range in energies from $10^{7} \mathrm{eV}$ to $10^{20} \mathrm{eV}$. Direct measurements from air-borne experiments can measure up to $10^{12} \mathrm{eV}$ of the primary particle energy [39] [40]. Figure 5.1] shows major components of the primary flux measured by different experiments [78] for primary energies below $10^{12} \mathrm{eV}$. For the primary cosmic rays at higher energies, indirect measurement is taken by observing the air showers using ground based detectors [41] 42]. Figure 5.2 shows the "all particle" spectra measured by a multitude of experiments for energies above $10^{11} \mathrm{eV}[78]$. 


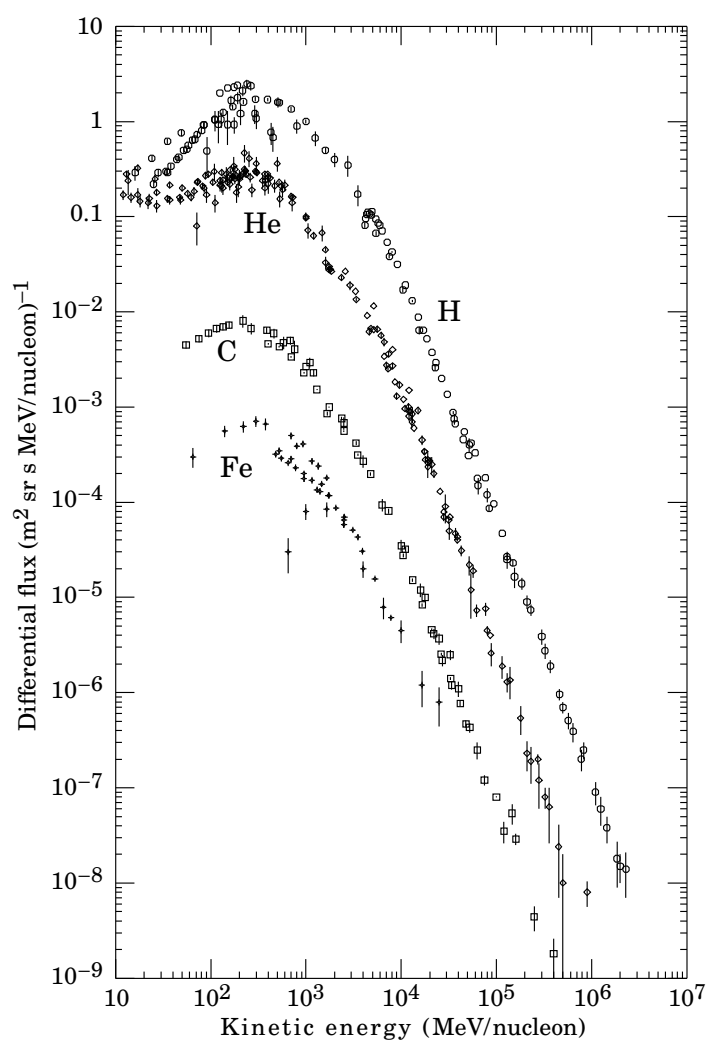

Figure 5.1: The cosmic ray spectrum for primary particle energies below $10^{12} \mathrm{eV}[78$.

The solar wind modulates the cosmic ray flux with energies below $10^{10} \mathrm{eV}$. For energies above $10^{10} \mathrm{eV}$, the spectrum follows a steeply falling power law spectrum in energy, $E^{-(1+\gamma)}$, where $\gamma$ is called the spectral index and it's value is $1.7 \pm 0.05$ [76]. For primary cosmic ray energies above $5 \times 10^{15} \mathrm{eV}$, the spectrum steepens to $\gamma \sim 2.0$. This is commonly known as the knee of the spectrum. Majority of the particles with energies below the knee originates from within the Milky Way galaxy. Above the primary energies of $\sim 10^{18} \mathrm{eV}$, the energy spectrum becomes flat. This feature of the spectrum is called 


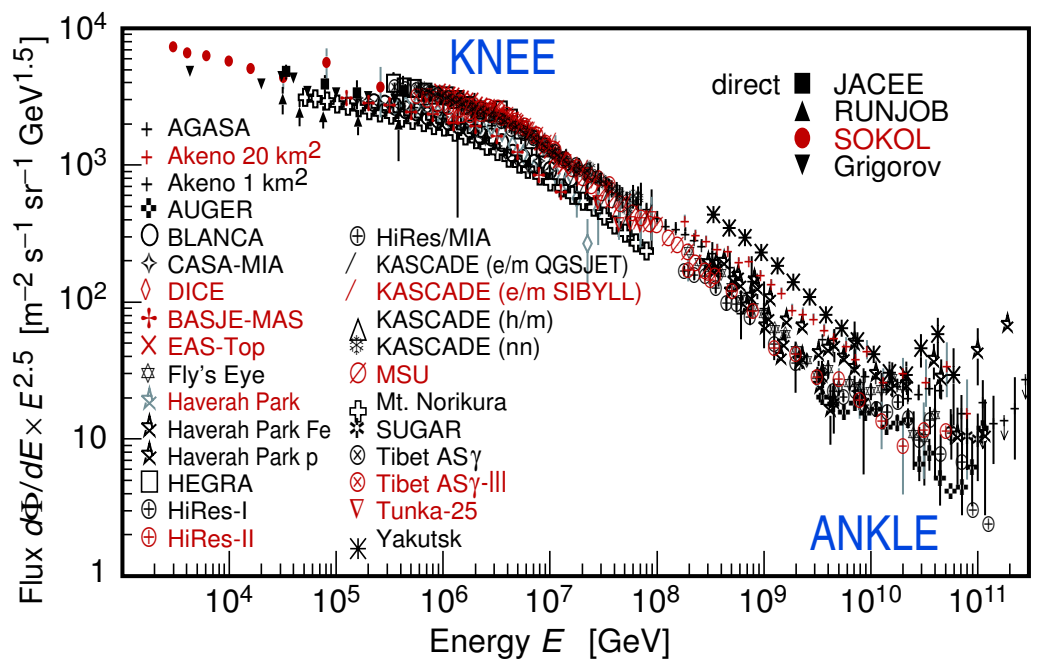

Figure 5.2: The "all particle" spectrum of cosmic rays with energies above $10^{11} \mathrm{eV}[78]$.

the ankle. Cosmic rays with energy in the ankle range originate from outside the galaxy, since the magnetic field of the galaxy can no longer confine the particles beyond energies of $\sim 10^{18} \mathrm{eV}$.

The primary cosmic ray particles interact in the atmosphere to produce the secondary cascades of pions and kaons. These pions and kaons themselves decay to produce a large number of cosmic ray muons. The production height is typically in the order of $20 \mathrm{~km}$ from the surface of the Earth. The energy spectrum and the angular distribution of the muons follow the primary spectrum. The flux of the cosmic ray muons has been measured my multiple experiments [43] 44] [45]. The muon spectrum also follows the steeply falling spectrum as the primary cosmic rays. The mean energy of the cosmic muons on the surface of the Earth is $4 \mathrm{GeV}$. The measured spectrum from different experiment agrees on the 
shape, however, there is a $20 \%$ disagreement in the measured value of the absolute flux.

The cosmic ray muons with a sufficiently high energy are able to penetrate down to the detector site in Soudan, MN. The flux for the muons drops off rapidly with depth as the threshold energy increases with increasing depth. The flux of the cosmic muons at the

Far Detector decreases by a factor of $\sim 10^{5}$ relative to the surface. The rate of incident muons at the detector site is $\sim 1 \mathrm{~Hz}$. The cosmic ray muons are important in validating and calibrating the far Detector. It is important to correctly simulate the muons.

\subsubsection{Simulation of Underground Muons}

The calculation of the cosmic ray muon flux at the Far Detector site involves propagating the cosmic muons flux measured at the surface through a digitized map of the rock overburden above the Soudan Underground Laboratory cavern. The muon flux at the surface is parameterized as follows [46]:

$$
N(E, \cos [\theta])=\frac{0.14 E^{-2.7}}{c m^{2} \mathrm{sr} \mathrm{GeV} \mathrm{s}}\left(\frac{1}{1+\frac{1.1 E \cos \theta}{115 \mathrm{GeV}}}+\frac{0.054}{1+\frac{1.1 E \cos \theta}{850 \mathrm{GeV}}}\right) .
$$

This parameterization is valid for $\theta<70^{\circ}$ when the curvature of the Earth is neglected and $\mathrm{E}>100 \mathrm{GeV}$ where the decay of the muons can be neglected. The first term in Equation 5.1 represents contributions from pions and the second term represent contributions from the kaons. To extrapolate the muon flux from the surface to the Far Detector, the information about the density and the composition of the local rock is required. The 
primary composition of the rock above the detector cavern site is known as lake Vermiliion Greenstone. The measured density of the rock is $2.8 \mathrm{~g} \mathrm{~cm}^{-3}$ [47]. The rock also contains a mixture of iron. Hence the average rock density varies with direction of the muon. The cosmic ray muon simulation for the MINOS Far Detector uses a rock density map developed by the Soudan 2 collaboration. The Soudan 2 rock map, combined with a digitized map of the surface topography, is used to calculate the overburden for a given muon direction [47]. The cosmic ray muon energy loss through the rock is parameterized as follows [46]:

$$
\frac{d E}{d x}=A+\frac{E}{L}
$$

The first term of the Equation 5.2 represents the energy losses from ionization, where $A \approx 1.9 \mathrm{MeV} / \mathrm{g} \mathrm{cm}^{-2}$. The second term accounts for the radiative energy losses, with $L \approx 2.5 \times 10^{5} \mathrm{~g} \mathrm{~cm}^{-2}$. In practice, however, $A$ and $L$ vary slowly with energy and $d E / d x$ undergoes stochastic fluctuations. Thus all three terms can be treated as constant. Thus using this approximation Equation 5.2 can integrated to give the $E_{X}$ by,

$$
E_{X}=\left(E_{o}+A L\right) e^{-X / L}-A L
$$

where $X$ is the depth considered, and $E_{o}$ is the surface energy. The flux at the surface level is used to calculate the flux at the Far Detector as a function of $E_{X}$ and $\theta$ with,

$$
N\left(E_{X}, X, \cos \theta\right)=N\left(E_{o}, 0, \cos \theta\right) d E_{o}=N\left(E_{o}, 0, \cos \theta\right) e^{X / L} d E_{X}
$$


The rock overburden $\mathrm{X}$ is determined for each value of $\theta$ using the Soudan 2 rock map. The surface energy $E_{o}$ is converted from $E_{X}$ using Eqn. 5.3. The flux at the Dar Detector site with the energy $E_{X}$ and the angle $\theta$ determined from the flux at the surface level with the energy $E_{o}$ and the angle $\theta$.

\subsubsection{Data to MC comparison}

The MINOS cosmic ray MC is generated in three steps. The first step is to select an initial energy and direction randomly for the muons using Equation [5.4 After which a random initial position for the propagation is chosen. The initial chosen positions are on an imaginary box placed around the detector. In the last step the muon is propagated to the Far Detector using the gminos package [48], and the detector response is simulated using the GEANT based [49] Photon Transport and the DetSim Package [50].

The data and the MC are compared using muons with well defined track length and fit. The events with only one track is kept. The tracks are required to cross 20 detector planes and have a length of 2 meters or more. The length cut is required to eliminate the neutron and the pion background. The muons with good fit to the track are selected $\left(\chi_{f i t}^{2} / n d f<1.5\right)$ to account for directionality (the zenith and the azimuth angle). The events passing these cuts have reliable track direction and reliable track fit. The cuts are applied to a large fraction of data and the MC. The selected events are compared for the distribution in number of planes crossed, in azimuthal distribution and the zenith angle distribution. 


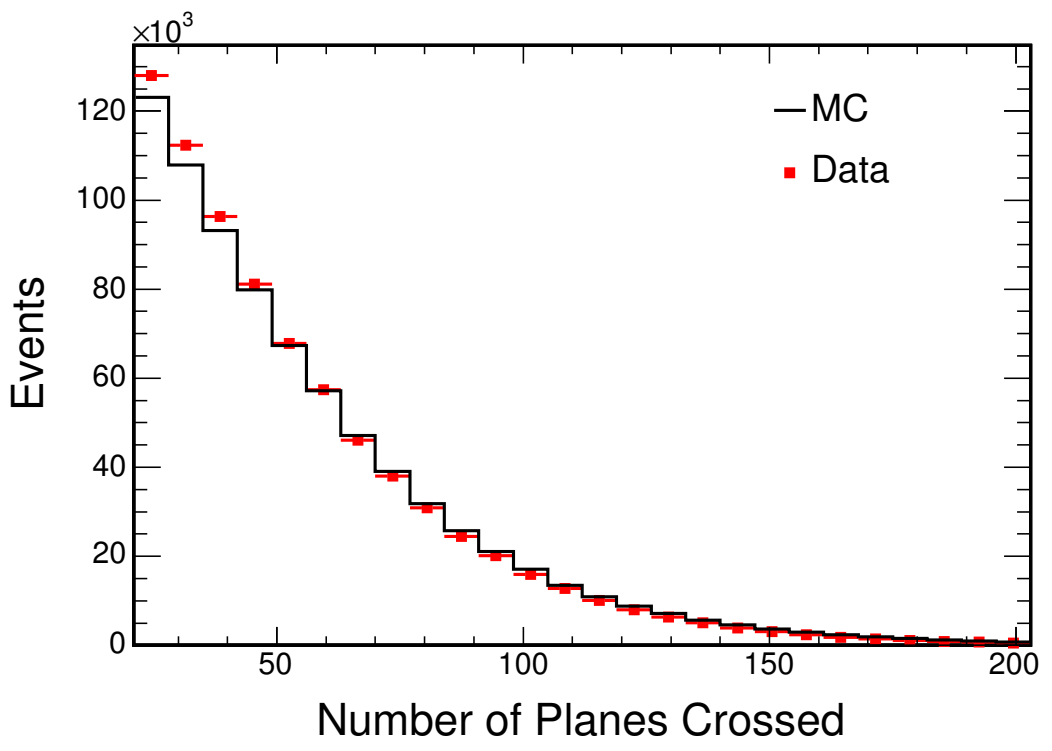

Figure 5.3: The muon track planes distribution.

The first distribution that is compared is the number of planes that the the muons cross. Figure 5.3 show the total number of detector planes crossed by the muons in the data and the MC. The distributions are normalized to give the same integral number of events. There is a good agreement between the data and the MC. The reconstructed azimuthal distribution of the data and the MC is shown in Figure 5.4. There is a general agreement between the data and the MC. The acceptance of the detector determines the shape of the distribution. The distribution around the azimuthal angles $70^{\circ}$ and $240^{\circ}$ are suppressed, because the trajectories near the X-Y plane, there are fewer number of events that satisfy the number of planes cut selection. The central "hump" in the azimuthal distribution $\left(65^{\circ}<\phi_{\text {azimuth }}<245^{\circ}\right)$ represents the events entering from the south side 


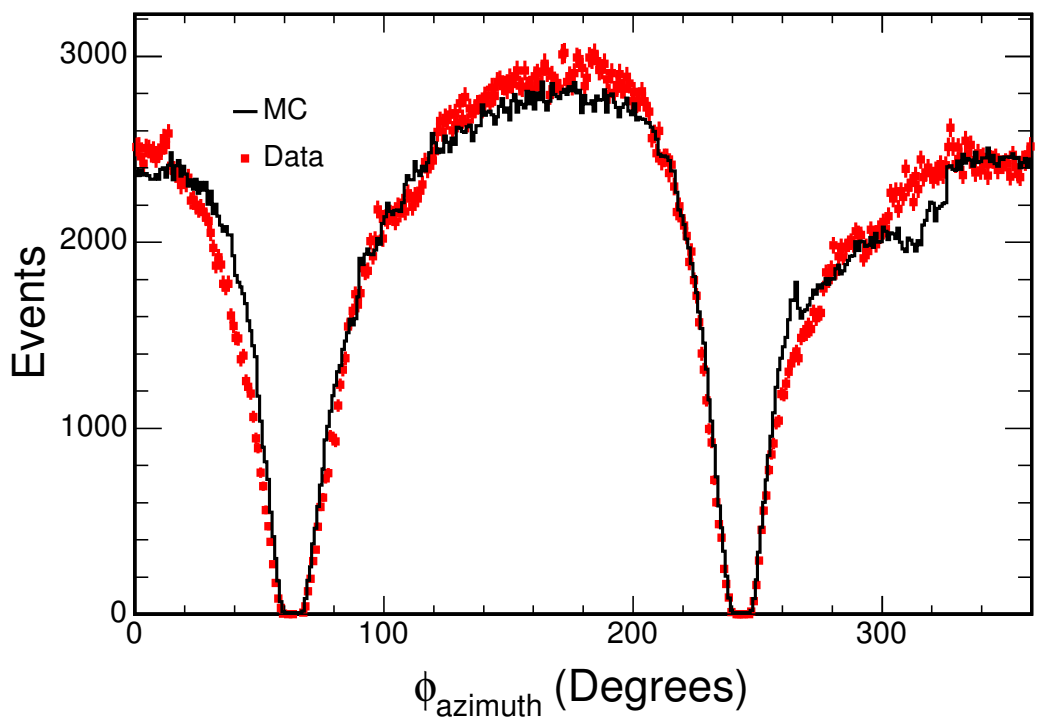

Figure 5.4: The distribution of the reconstructed azimuthal angle.

of the detector. While the events entering from $\phi_{\text {azimuth }}<65^{\circ}$ and $\phi_{\text {azimuth }}>250^{\circ}$ are muons coming from the north side of the detector. There are more events entering from the north side of the detector than the south side. This is because the muons have to cross more rock coming from the north than the south. In the simulation, the shape of the azimuthal distributions of the data and the $\mathrm{MC}$ from the north at $\phi_{\text {azimuth }}=330^{\circ}$ do not agree. This is because the east and the west overburden profiles in the simulation have been reversed. This effect is also seen and noted by other members of the MINOS collaboration [32] [51. The MC has a spike around around $\phi_{\text {azimuth }}=260^{\circ}$, while the data does not. This could be due to the slight difference in the position of the Soudan 2 detector, from which the rock map was used, and the MINOS Far Detector. 


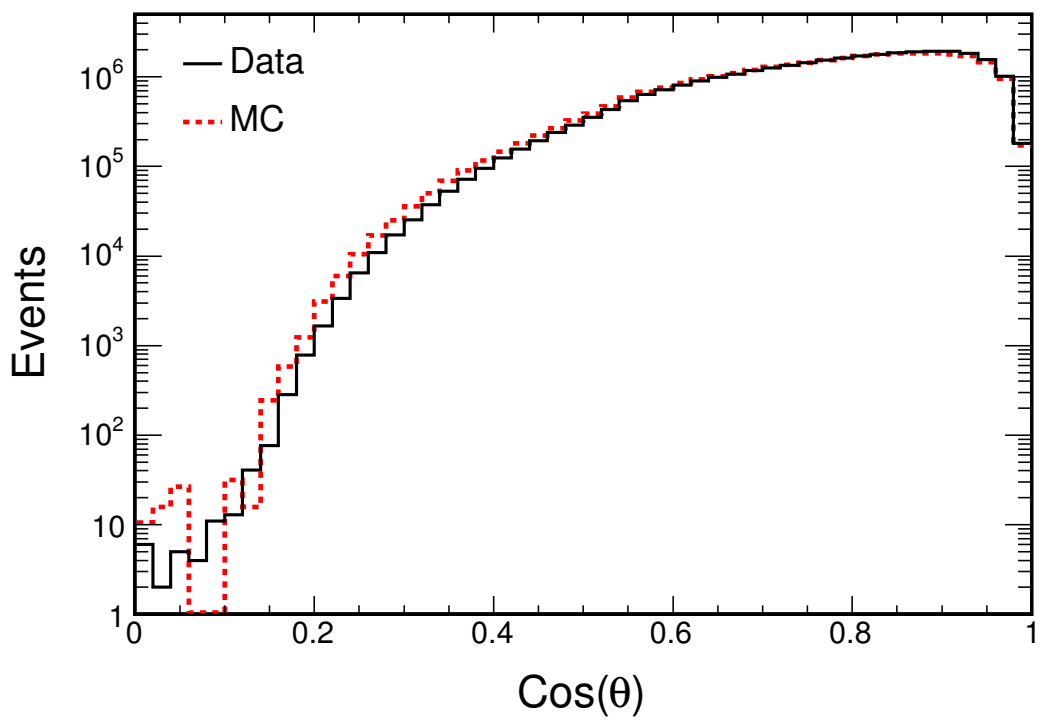

Figure 5.5: The distribution of the reconstructed cosine of the zenith angle.

The zenith angle distribution of the data and the MC is shown in Figure [5.5. The MC distribution is scaled to gave the same integral number of events as the data. The $\mathrm{MC}$ agrees with the $\mathrm{MC}$ within a few percent for $0.6<\cos \theta<0.9$. The $\mathrm{MC}$ simulation over estimates the data for $0.2<\cos \theta<0.5$ by over $10 \%$. Near the horizon, the MC over estimates the data by a factor of two. This discrepancy between the data and the MC could be attributed to the simplicity of the MC modeling. The Equation 5.1] puts a hard low energy cutoff for the allowed muon energy as a function of the muon direction. The actual energy required for the muons on the surface to propagate to the given distance is not a hard threshold. This needs to be addressed in the simulation for a proper agreement between the data and the MC. 
The cosmic ray muons are important to the the MINOS Far Detector in understanding the detector response and the rock surrounding the detector. The simulation of the cosmic rays agrees with the data well demonstrating that the detector response is well understood. In the following section the neutrino induced muons are discussed.

\subsection{Atmospheric Neutrinos}

The atmospheric neutrinos are created as a result of the parent meson decays and the muon decays in the atmosphere. In this section an analytical description of the atmospheric neutrino flux is given, with the description of the uncertainties in the flux. The description of the simulation of the neutrino induced muons is also included. The neutrino cross section and the neutrino to anti-neutrino cross section ratio is discussed as well.

\subsubsection{Atmospheric Neutrino Flux}

The following is a summary of the flux calculations done by T. Gaisser and M. Honda [52]. The neutrino flux $\phi_{\nu_{\alpha}}$ for a neutrino flavor $\alpha$ is given by

$$
\phi_{\nu_{\alpha}}=\phi_{p} \otimes R_{p} \otimes Y_{p \rightarrow \nu_{\alpha}}+\sum_{A} \phi_{A} \otimes R_{A} \otimes Y_{A \rightarrow \nu_{\alpha}}
$$

where $\phi_{p}$ is the flux of the primary cosmic ray protons coming from outside the geomagnetic field, $R_{p}$ is the filtration of the low energy particles by the geomagnetic field, and $Y_{p}$ is the yield of neutrinos per primary cosmic ray protons. The subscript $A$ stands for the atomic 
mass of the primary particles. The primary cosmic rays of low energies are prevented from entering and interacting in the atmosphere by the presence of the geomagnetic field. The threshold energy for the primary cosmic rays that are blocked by the magnetic field varies with the detector's geomagnetic latitude. The threshold energy increases with decreasing geomagnetic latitude.

The atmospheric neutrinos are produced primarily from the two body decays of the charged pions and kaons into muons. The muons in turn decay to give electrons and neutrinos,

$$
\begin{aligned}
\pi^{ \pm} & \rightarrow \mu^{ \pm}+\nu_{\mu}\left(\bar{\nu}_{\mu}\right) \\
\text { and }, \mu^{ \pm} & \rightarrow e^{ \pm}+\nu_{e}\left(\bar{\nu}_{e}\right)+\bar{\nu}_{\mu}\left(\nu_{\mu}\right)
\end{aligned}
$$

with a similar chain for the kaon decays. Assuming that all the particles (mesons, and muons) decay, we expect,

$$
\begin{aligned}
\frac{\nu_{\mu}+\bar{\nu}_{\mu}}{\nu_{e}+\bar{\nu}_{e}} & \sim 2, \\
\frac{\nu_{\mu}}{\bar{\nu}_{\mu}} & \sim 1, \text { and } \\
\frac{\nu_{e}}{\bar{\nu}_{e}} & \sim \frac{\mu^{+}}{\mu^{-}} .
\end{aligned}
$$

The flux of $\bar{\nu}_{\mu}$ and $\nu_{\mu}$ are approximately the same because the muon decay produces a $\bar{\nu}_{\mu}$ and the meson decay produces a $\nu_{\mu}$. In addition, the kinematics of the $\pi$ and the $\mu$ decay 
are such that neutrinos in each chain carry approximately the same amount of energy [52].

An analytic approximation of the neutrino flux can be made for the neutrinos of energies greater than $1 \mathrm{GeV}$. This is a good approximation for the primary energy power law spectrum and assumes inclusive cross sections that obey the Feynman scaling in the fragmentation region [52]. The differential flux of the neutrinos from the meson decay is given by,

$d N\left(E_{\nu}, \theta\right) / d E_{\nu}=\frac{\phi_{N}\left(E_{\nu}\right)}{\left(1-Z_{N N}\right)(1+\gamma)}\left\{\left[\frac{Z_{N \pi}\left(1-r_{\pi}\right)^{\gamma}}{1+B_{\pi \nu} \cos \theta E_{\nu} / \epsilon_{\pi}}\right]+0.635\left[\frac{Z_{N K}\left(1-r_{K}\right)^{\gamma}}{1+B_{K \nu} \cos \theta E_{\nu} / \epsilon_{K}}\right]\right\}$,

where, $\phi_{N}\left(E_{o}\right)=d N / d E_{i}=A \times E_{i}^{-(1+\gamma)}$ is the differential primary spectrum of energy $E_{i}$, with $\gamma=1.7$ and $A=1.8$ [46]. The neutrino flux in Equation [5.9] is proportional to the primary spectrum for the neutrino energies in consideration. The $r_{\pi}$ and the $r_{K}$ are constants, and are defined as the square of the ratio of the muon mass to the meson mass, $\theta$ refers to the zenith angle of the neutrinos. $B_{N(\pi, K)}$ are constants, and are defined by 46]

$$
B_{N_{j}}=\left(\frac{\gamma+2}{\gamma+1}\right)\left(\frac{1}{1-r_{j}}\right)\left(\frac{\Lambda_{j}-\Lambda_{N}}{\Lambda_{j} \ln \left(\Lambda_{j} / \Lambda_{N}\right)}\right)
$$

where $\Lambda_{j}$ is the attenuation length of the mesons $(\pi$ and $K)$, and $\Lambda_{N}$ is the attenuation length of the primary nucleon. The $\epsilon(\pi, K)$ are the critical energies in GeV below which the energy loss of the mesons and their decays can be neglected [46]. The $Z_{N \rightarrow \pi(K)}$ is the spectrum weighted moments that contains the physics of production of the parent pions and the kaons. 
The flux from Equation 5.9 becomes more accurate as the neutrino energy increases from $1 \mathrm{GeV}$. The energy and the angular dependence of the neutrinos are determined by the competition between the decay interaction in the atmosphere of the parent pions and kaons. From Equation [5.9] the neutrino spectrum follows the same power law as the production spectrum of the parent mesons for $\left.E_{\nu} \ll \epsilon_{(} \pi, K\right) / \cos \theta$. On the other hand, when $E_{\nu} \gg \epsilon(\pi, K) / \cos \theta$, the power spectrum is steeper and is proportional to $\sec \theta$. The spectrum of neutrinos from the pion decays steepens before the spectrum from the kaon decays 52 . Thus with an increase in the neutrino energy, the kaons become more important. Figure [5.6 shows the relative contributions from the parent mesons to the

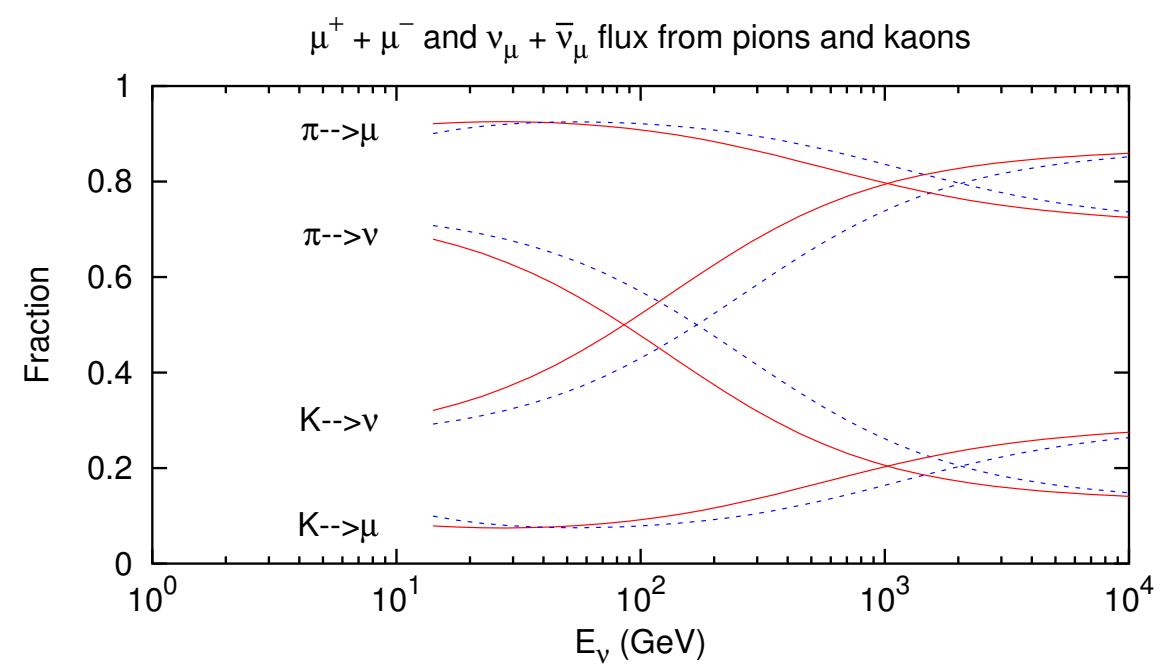

Figure 5.6: The parent mesons' fractional contribution to the muon flux and the atmospheric neutrino flux. The solid lines are for the vertical flux, and the horizontal lines are for $\theta=60^{\circ}[52$.

cosmic ray muons fluxes and the atmospheric neutrino fluxes as a function of energy [52]. 
For the pions, the vertical direction is represented by the solid line, the $\theta=60^{\circ}$ curve is represented by the dashed line. The reverse is true for the kaons.

\subsubsection{Uncertainties in the Flux Calculations}

The largest uncertainty in the flux calculations of the atmospheric neutrinos come from the primary spectrum and from the treatment of the hadronic interactions [52]. Recent measurements by the AMS experiment [53] and the BESS [54] show that the uncertainty in measurements of the primary spectrum is $\pm 5 \%$ below $100 \mathrm{GeV}$ per nucleon and $\pm 10 \%$ at $10 \mathrm{TeV}$ per nucleon [52]. The estimate on the uncertainty was done fitting a power law spectrum to the AMS and the BESS data to extrapolate the error beyond the upper limits of the measurements $(\approx 100 \mathrm{GeV})$. However, an extensive estimate of the primary spectrum can be done using all valid measurements. A collection of data from different experiments, gathered by Gaisser and Honda [52, for primary protons and helium is shown in Figure 5.7 The figure includes measurements below $100 \mathrm{GeV}$. There is a lack of measurement in the $\mathrm{TeV}$ range that is important for the neutrino induced muons. If the high energy data is renormalized to the low energy data, the uncertainties are $\pm 20 \%$ below $20 \mathrm{GeV}$, and $\pm 30 \%$ above.

The uncertainties due to the hadronic interactions are more difficult to estimate. The phase space for proton-air interactions that contribute to the neutrino signals are not well measured. There is an overall spread of $\pm 20-25 \%$ in the data for the hadronic interactions [52]. When the uncertainties from the primary spectrum and the hadronic interactions 

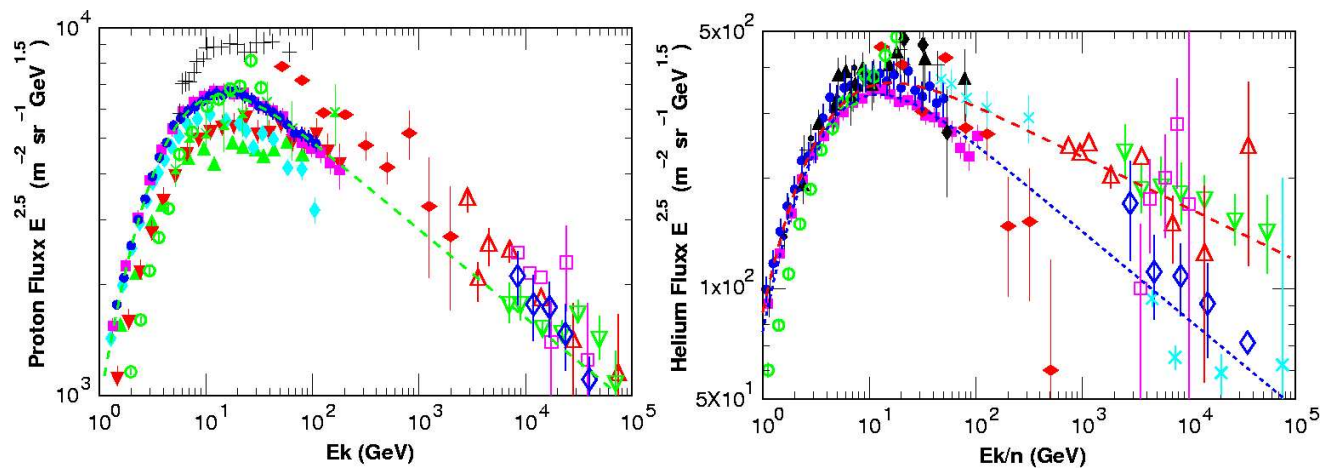

Figure 5.7: The observed flux for the proton and helium cosmic ray primaries. The dashed lines show the extrapolated fits. In the data set: Weber [55], crosses LEAP [56], upward solid triangles; MASSI [57], open circles; CAPRICE [58], vertical solid diamonds; IMAX [59], downward solid triangles; BESS98 [54], solid circles; AMS [53], solid squares; Ryan 60], horizontal solid diamonds; JACEE [61], downward open triangles; Ivanenko 62], upward open triangles; Runjob [63], open diamonds; Kawamura 64, open squares.

are combined assuming that they were independent, the overall theoretical uncertainty for the relevant range for the neutrino interactions is $\pm 20-25 \%$ [52]. For this oscillation analysis done with neutrino induced muons, a conservative $\pm 25 \%$ uncertainty is assumed in Chapter 7

\subsubsection{Simulation of the Neutrino Induced Muons}

For the oscillation analysis described in Chapter 7 the simulation for the neutrino induced muons was done using the Nuance neutrino generator [70]. The flux for the neutrino induced muons were generated by BARTOL96 flux tables [71]. The parton distribution functions were generated by GRV94 [72]. The primary Nuance MC program generates a list of events in the rock surrounding the MINOS Far Detector with the events' kinematic 
information for a given detector exposure. The kinematic information includes the parent neutrino energy, it's initial zenith angle, and the interaction vertex, and the momentum in the $x, y$ and the $z$ direction. Nuance also records the kinematic information about the daughter muon, for example, it's initial energy and the zenith angle, it's initial $x, y$ and the $z$ coordinates, and initial momentum in the $x, y$ and the $z$ direction. Nuance also propagates the generated muons from the neutrino interaction vertex position to Far Detector. The program then records the neutrino induced muon's kinematic information at the time of it's arrival at the detector.

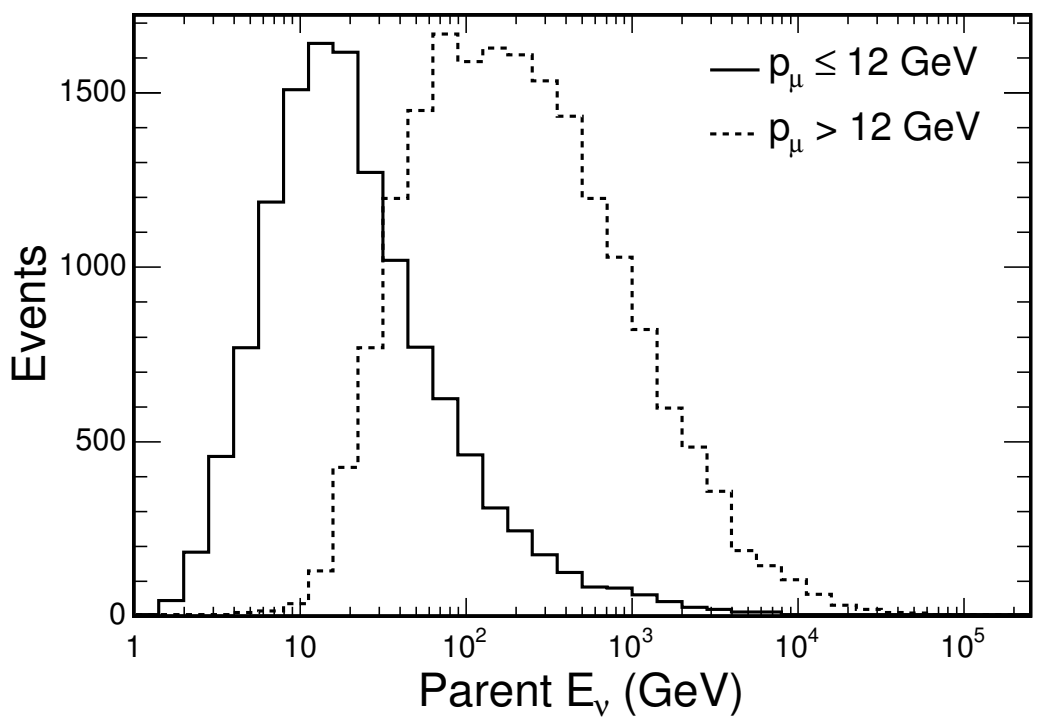

Figure 5.8: Parent neutrino energies of muons with measured momenta $\leq 12 \mathrm{GeV}$ and measured momenta $>12 \mathrm{GeV}$. The neutrino induced muons were generated by using the Nuance neutrino generator [70].

The kinematic information about the parent neutrinos and the daughter muons are 
fed to the GEANT [49] based gminos Package [48, along with the Photon Transport and the DetSim Package [50] that propagate the daughter muons through the Far Detector. The output for this process is run through Standard Reconstruction software to generate the final neutrino induced candidate muon files. A total of $60 \mathrm{~K}$ events were generated by the final reconstruction. This is an equivalent detector exposure of 400 years.

The energy and the zenith angle distribution of these neutrino induced muons are discussed in Chapter [7. Figure [5.8 shows the parent neutrino energy for the muons with measured momenta $12 \mathrm{GeV}$ or less, and events with measured momenta greater than 12 $\mathrm{GeV}$. The high momentum $\left(p_{\mu}>12 \mathrm{GeV}\right)$ muons come from the high energy neutrinos. Most of the muon momenta are above $\sim 3 \mathrm{GeV}$.

\subsubsection{Neutrino Cross Sections}

The total neutrino cross section can be considered as the combined effect of the three channels, quasi-elastic (QE), single pion production $(1 \pi)$, and the deep inelastic scattering (DIS) that produces multiple pions,

$$
\sigma_{\nu(\bar{\nu})}=\sigma_{Q E}+\sigma_{1 \pi}+\sigma_{D I S}
$$

Figure 5.9 shows the total cross sections for charged current (CC) neutrino interactions [65. The solid line indicates the total cross sections, the dotted line indicates DIS interactions, the dashed line indicates QE interaction, and the dashed dotted line indicates $1 \pi$ interactions. Several measurements are superimposed on the plots as well. The cross 


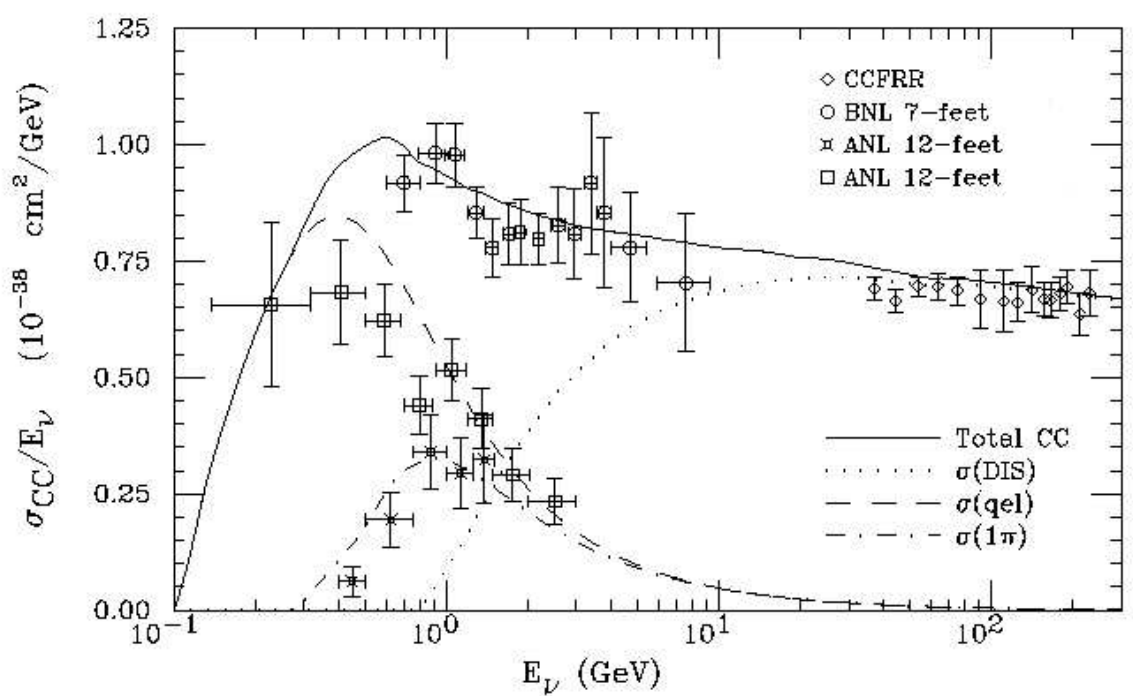

Figure 5.9: Neutrino cross sections [65]. For the data: CCFRR, 66]; BNL, 67]; and ANL 68 and 69.

sections from the DIS scattering dominates for $E_{\nu}$ above $2 \mathrm{GeV}$ or so. As seen in Figure 5.8. most of the parent neutrino energies from the simulated neutrino induced Monte Carlo sample are above $3 \mathrm{GeV}$. This indicates that most of the neutrino interactions in the surrounding cavern rock are DIS interactions.

Figure 5.10 shows the ratio of the total charged current cross section to neutrino energy for neutrinos and anti-neutrinos as a function of neutrino energy [73]. Both the statistical and the systematic uncertainties are included for the errors. The dashed lines indicate the average values for all measurements. The average ratio for neutrino is $0.677 \pm 0.014$, and the average ratio for the anti-neutrino is $0.334 \pm 0.008$ [73].

The ratio of the neutrino flux to the anti-neutrino flux is important for the relative 


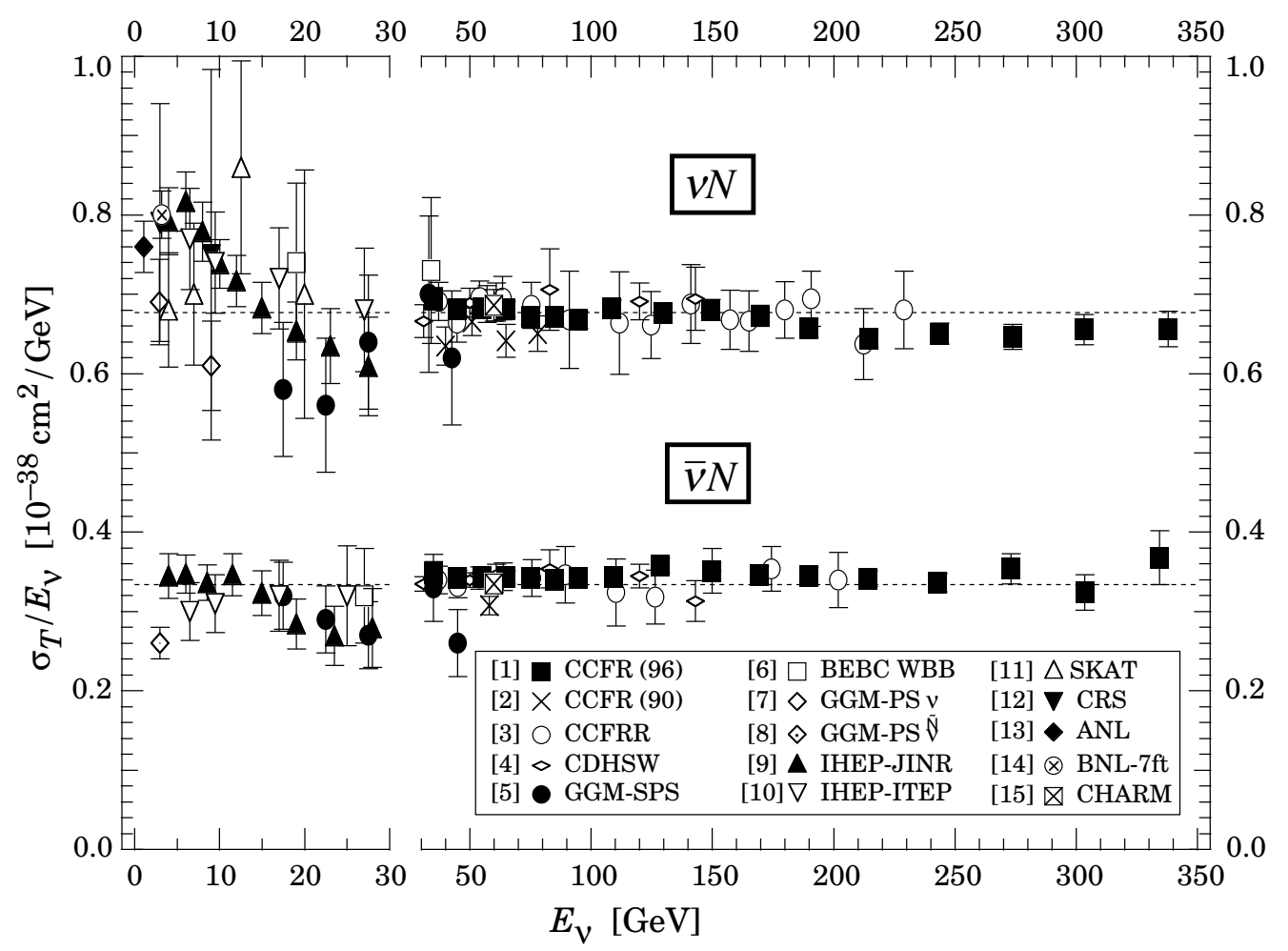

Figure 5.10: The ratio of the total neutrino cross section to the neutrino energy [73].

flux of the $\nu_{\mu}$ and the $\bar{\nu}_{\mu}$ since the the uncertainty in the cross sections directly affects the number of neutrino induced muons $\left(\mu^{-} \& \mu^{+}\right)$in the detector. The ratio of the neutrino to anti-neutrino cross section rises to 3.5 at $\sim 700 \mathrm{MeV}$ and decreases with increasing energy. The ratio becomes constant around 2 for $E_{\nu}>3 \mathrm{GeV}$. Since most of the primary neutrino energies are above $3 \mathrm{GeV}$, the systematic uncertainties for the oscillation analysis as well as the CPT charge ratio analysis done in Chapter 7 should be relatively small. 


\section{Chapter 6}

\section{Data Selection}

\subsection{Introduction}

The construction of both the Super Modules and the Veto Shield ended in July 2003. The data analyzed here were taken from August 1, 2003, to April 30, 2006 - live time of 890 days $\left(\sim 13 \mathrm{kT}\right.$ years). A total of $4.11 \times 10^{7}$ events were recorded. This chapter describes the selection and efficiency of the data selection of these events, accounts for possible sources of background, and describes the distribution of selected events. A description of the systematic uncertainties due to the selection cuts is also included.

\subsection{Selection Cuts and Efficiencies}

The cuts used to select neutrino induced muons were developed for the cosmic ray muon study 32]. All data analyzed here were reconstructed with Standard Reconstruction R1.14 
developed by the MINOS core software group. In the reconstruction, various algorithms are used to processes raw data from the Far Detector in order to determine the track qualities like length, timing, momentum, and charge. The information is recorded at both track level and strip level. At the track level, the information about the track can be easily accessed and it refers to the properties of the entire track. The reconstruction determines over 150 track related quantities useful for this analysis including number of planes crossed, track length, number of timing digits, timing goodness to fit $\chi^{2}$, track goodness to fit $\chi^{2}$, vertex position and direction. At the strip level, the information about the individual strip hits including the track's strip timing and positions are recorded. To access strip level information the code iterates over the entire track strip data. This allows the user to customize the analysis, and extract information about a particular analysis that may not have been recorded at the track level.

A series of nine cuts at the track level are first applied to the reconstructed data. These cuts are also applied to the cosmic ray Monte Carlo sample to compare the selection efficiencies of the simulated muons, and to the neutrino induced Monte Carlo sample to select good neutrino induced Monte Carlo events. The function of these cuts is to select reliable muon events for the strip level analysis. These cuts select the events with proper demultiplexing, as well as events with a single muon track. The cuts also select tracks with enough length to be muon candidates, tracks with a good fit to the timing and to the hit positions, and the tracks with vertex positions in the allowed regions of the detector. This eliminates approximately $48 \%$ of the total events recorded in the reconstructed files. 
Events passing the track level cuts are analyzed at the strip level. These strip level cuts ensure that the track fit distributions of the data and the Monte Carlo sample agree with each other. The timing cuts examine the strip timing from both the east and west sides of the detector in order to have reliable timing as a whole. The timing cuts also determine the fit in order to the timing in the $y$ direction to allow only the clean up or down going events. Finally the beam events coming from the NuMI beam in Fermilab near Chicago, Illinois, are removed by comparing the beam spill time and the event trigger time.

Table 6.1 shows the selection efficiencies of the cuts imposed to the data and the Monte Carlo (MC) simulations. There are two important differences between the data and the MC selection efficiency. The first difference is in demultiplexing. In general the MC samples have cleaner reconstructed hits than the data and hence have higher demultiplexing efficiency. The second important difference is that neither the Cosmic Ray MC nor the neutrino induced MC sample has simulated multiple muons. Thus the number of events identified as multiple muons removed by the reconstruction is small in the MC, with any such events being poorly reconstructed single muons. It is then more useful to normalize the efficiencies after the "Number of Tracks" cut (2), since the relative efficiencies become more compatible. The differences between the data and the Monte Carlo samples are used for determining the systematic uncertainty due to the selection cuts. 
Table 6.1: Selection efficiency for the data, cosmic ray MC, and neutrino induced MC samples

\begin{tabular}{|l|c|c|c|}
\hline \hline & Data & Cosmic MC & Neutrino Induced MC \\
\hline Total Events & $4.11 \times 10^{7}$ & $1.04 \times 10^{7}$ & $5.77 \times 10^{4}$ \\
\hline Total Tracks & $3.41 \times 10^{7}$ & $8.94 \times 10^{7}$ & $5.29 \times 10^{4}$ \\
\hline Total Tracks (fractional) & 1.0 & 1.0 & 1.0 \\
\hline \hline 1. Demultiplexed & 0.83 & 0.86 & 0.91 \\
\hline 2. Number of Tracks & 0.78 & 0.86 & 0.91 \\
\hline 3. Number of Planes & 0.58 & 0.65 & 0.72 \\
\hline 4. Track Length & 0.58 & 0.65 & 0.70 \\
\hline 5. Track Time Digits & 0.55 & 0.63 & 0.68 \\
\hline 6. $\chi_{\text {time }}^{2} /$ ndf & 0.55 & 0.63 & 0.68 \\
\hline 7. $\chi_{\text {fit }}^{2} / n d f$ & 0.54 & 0.61 & 0.67 \\
\hline 8. Vertex Y Reconstruction & 0.54 & 0.61 & 0.66 \\
\hline 9. Contained Vertex & 0.52 & 0.59 & 0.65 \\
\hline 10. East \& West Side Timing & 0.52 & 0.59 & 0.63 \\
\hline 11. Time vs. Y Correlation & 0.52 & 0.59 & 0.62 \\
\hline 12. Directional Consistency & 0.51 & 0.58 & 0.61 \\
\hline 13. $\chi_{\text {new-fit }}^{2} /$ ndf & 0.49 & 0.57 & 0.60 \\
\hline 14. Beam Events & 0.49 & 0.57 & 0.60 \\
\hline
\end{tabular}

\subsubsection{Track Level Cuts}

\section{Demultiplexing}

The MINOS Far Detector has been designed such that eight equally spaced fibers in a scintillator plane are optically summed. This means that the signal received by one pixel in the photo-multiplier tube could have come from any one of the eight scintillator strips. Information read out by the electronics is therefore multiplexed. The first step in the reconstruction process is thus demultiplexing. The process of demultiplexing is quite complex, however, the basic algorithm for demultiplexing a track is done by matching the 
pattern of the hits from the east and west sides of the Far Detector. Details of the demultiplexing algorithm can be found in the reference [32]. Tracks failing the demultiplexing stage of reconstruction are unreliable and are kept out of the analysis. This removes $17 \%$ of the track events from the data. The the fractional events passing the demultiplexing cut for the Cosmic Ray Monte Carlo sample and the neutrino induced Monte Carlo sample is somewhat higher. This is because the simulations in general have cleaner recorded hits than the actual data.

\section{Multiple Muons}

A small fraction of events in the Far Detector are multiple cosmic ray muon events. These muons are created in the atmosphere from the decay of pions and kaons from the same interaction of the primary cosmic ray nuclei. Although multiple events are of interest in cosmic ray physics, events that are tagged as multiple muons are not useful to this analysis and are therefore not considered. As seen in Table 6.1 this cut reduces the number of events in the data by $5 \%$ and has no effect on the Monte Carlo simulations. This is because neither the Cosmic Ray Monte Carlo sample nor the neutrino induced Monte Carlo sample has multiple muons in the model.

\section{Event Length}

There are two selection cuts that ensure that events have a long enough track to be well reconstructed muons. The first cut requires the number of planes crossed by the event to 
be twenty or greater. Depending on the track direction, the events passing this cut can range from 1.2 meters up to 8.1 meters in length. The second length cut requires that the length of the track itself be 2 meters or more. The length cuts together eliminate events that have poor reconstruction or are short pion tracks. Another advantage of requiring a longer track length is that the events will generally have a higher number of timing digits that are used to determine the directionality (up/down) of the muons which is discussed in the following section.

\section{Timing}

To distinguish the upward going events (neutrino induced muons) from the downward going events (mostly cosmic ray muons) it is essential to have a good timing measurement. The first timing cut requires that the of number timing digits in a track be greater than or equal to 32 . One way to observe the effect of this cut is to compare the measured inverse beta distribution with and without the cut. The inverse beta, or $1 / \beta=c / v$, is the ratio of the measured velocity $(v)$ of the muon to the speed of light $(c)$. The downward going muons have a negative value for $1 / \beta$ and upward going muons have a positive value for $1 / \beta$. Events with a higher number of timing digits that are used to calculate the muon's velocity should, in principle, have a better separation of upward to downward going muons in the measured $1 / \beta$ distribution. This is illustrated in the $1 / \beta$ spectrum for the data in Figure 6.1, which shows the distributions with and without the timing cut imposed. The inverse beta values for the downward going and the horizontal muons are negative. The 
distribution without the timing cut has a long positive tail creating a large background for the upward going events with positive inverse beta values. The shaded plot represents the

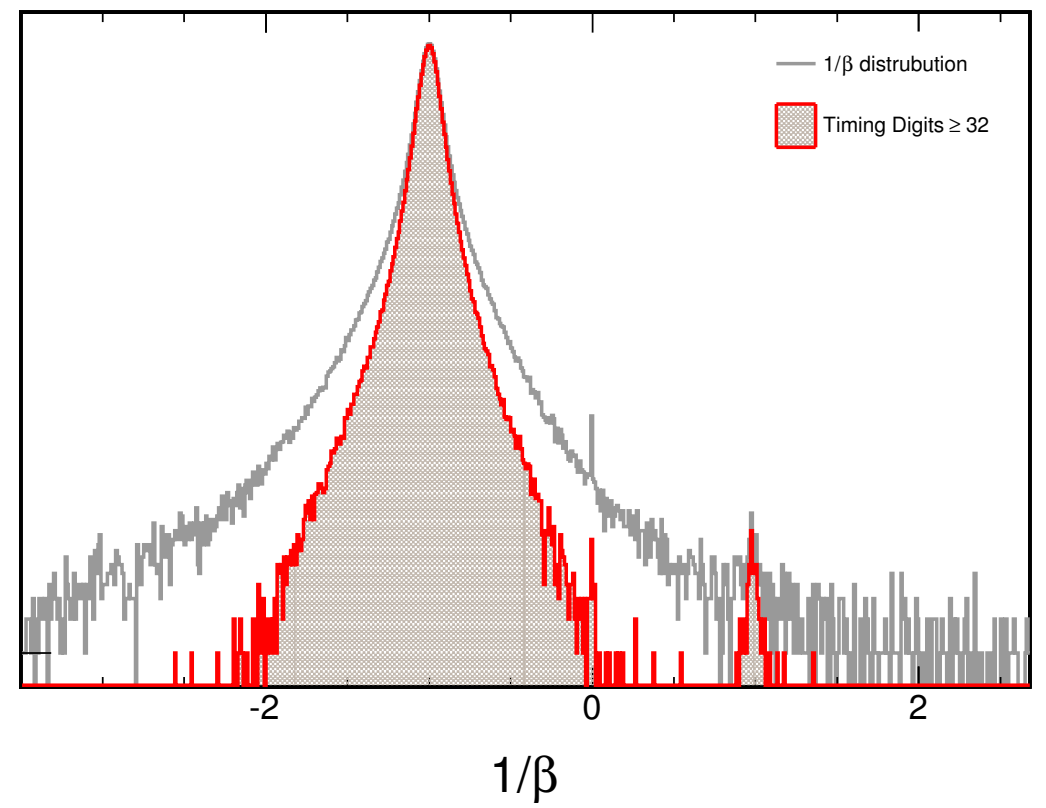

Figure 6.1: $1 / \beta$ distribution for data with and without the timing number of digits cut.

distribution with the timing cut added. As a result the positive $1 / \beta$ values, representing upward going candidates, are seen to be better separated from the downward going events with negative $1 / \beta$ values. Also visible is a small peak around zero for both distributions. The events in this region $(|1 / \beta| \simeq 0)$ have poor timing in general, and will be dealt with later by examining the timing information at the strip level. An additional timing cut described in Section 6.2.2 helps eliminate a large fraction of this peak.

The second timing cut sets the goodness to fit $\chi^{2} / n . d . f$. for the event timing in the $\mathrm{y}$ 
direction at 3 or less. This is a conservative cut that allows a greater number of events to pass the track level cuts while excluding events with extremely poor timing. The combined effect of the two timing cuts does not have any effect on the efficiency. This is because the length cuts (cuts 2 and 3 from Table 6.1) eliminate the majority of events with lower a number of timing digits, and tracks with poor fit to the timing digits.

\section{Track Fit - Track Level}

The track fitting package in the standard reconstruction software identifies the tracks' momentum and charge by a track finder that uses the principle of the Kalman filter [75]. The Kalman filter is a set of equations that iteratively estimate the state of a process in order to minimize the mean of the squared error for the track hits. For MINOS track fitting, the filter accounts for correlated errors like energy loss and multiple scattering, providing an accurate description of the muon track. The tracks with fit $\chi^{2} /$ n.d.f. $<2.3$ are kept for analysis. Figure 6.2 shows the $\chi^{2} / n . d . f$. distributions for the data and the Monte Carlo sample normalized to have the same integral number of events. The shape of the data distribution appears to be broader than that of the Monte Carlo sample. This is perhaps due to the fact that the simulations contain cleaner hits and the reconstruction fits the tracks better, thus the distribution is narrower around the peak for the Monte Carlo sample. The difference in the track fit distribution will contribute to the systematic uncertainty in the selection process, and will be taken into account in Section 6.5. 


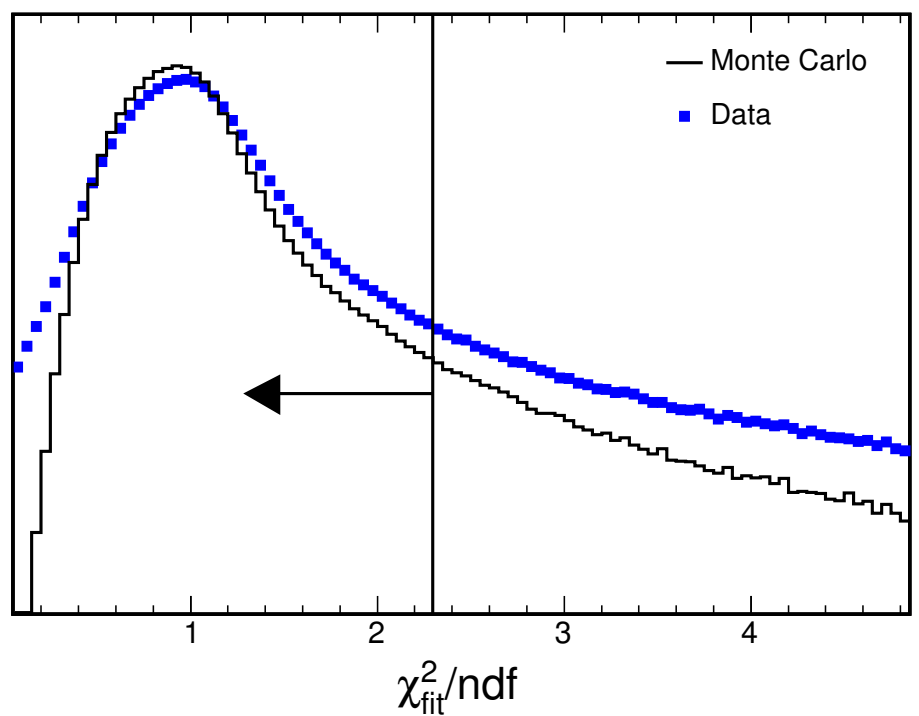

Figure 6.2: Track fit $\chi^{2} /$ n.d.f. distributions for the data and the Monte Carlo sample. The plots are scaled such that both samples have the same integral number of events. The vertical axis has a log scale.

\section{Track Vertex}

The vertex cuts consist of determining the proper track vertex direction as well as the track vertex position. The vertex direction cut removes events with incorrectly reconstructed vertices in the $y$ direction (cut 8 from Table 6.1). This is done by taking the vertex $y$ position and dividing it by its absolute value. This is then multiplied by the ratio of vertex direction cosine to its absolute value. The final product should yield \pm 1 , and +1 if the vertex direction is reconstructed properly. There are two vertex position cuts. The first cut ensures that the track vertex is less than 4.4 meters from the center axis of the detector. This eliminates events with unphysical vertex positions. The second cut requires 
that the track vertex position be outside the 3.7 meter fiducial radius from the center of the detector, and be within $15 \mathrm{~cm}$ from either the front or the back face of the detector. Events failing this cut are potentially contained atmospheric neutrino events.

\subsubsection{Strip Level Cuts}

\section{Timing}

Strip level timing is analyzed by extracting the strip timing from both the east and west sides of the detector. The first cut requires that the time information is available on both the east and west strip ends, and the slopes of time in the $y$ direction have the same sign on both ends. Events failing this cut (number 10 from Table 6.1) have unreliable timing and are kept out of the analysis.

The timing correlation and slope for the strip timing in the $y$ direction are also calculated using the strip timing. In the detector coordinates, positive $y$ points upward, away from the center of the Earth. The correlation, $r$, between time $(t)$ and $y$ scatter is given by the standard formula,

$$
r=\sqrt{\frac{S S_{t y}^{2}}{S S_{y y} S S_{t t}}}
$$

where,

$$
\begin{aligned}
S S_{y y} & =\sum_{i}\left(y_{i}-\bar{y}\right)^{2} \\
S S_{t t} & =\sum_{i}\left(t_{i}-\bar{t}\right)^{2}
\end{aligned}
$$




$$
S S_{t y}=\sum_{i}\left(y_{i}-\bar{y}\right)\left(t_{i}-\bar{t}\right)
$$

where, $\bar{t}$ and $\bar{y}$ are the mean of the time and $y$ positions, respectively. Muon tracks with $|r| \geq 0.4$ are kept (number 11 from Table 6.1). With a low coefficient of correlation in the timing, the track's $y$ directionality is ambiguous. This is illustrated in Figure 6.3, where
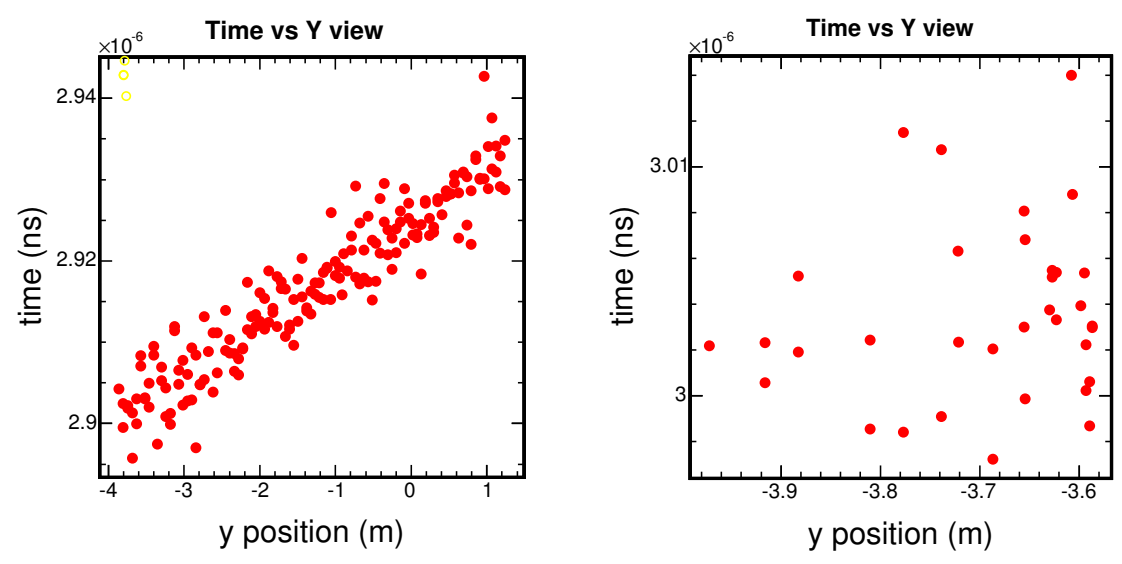

Figure 6.3: Time vs. Y scatter plot for two different events.

the plot on the right has a poor correlation $(\sim 0)$ in the time vs. $y$ scatter, and it is not obvious whether the muon is upward or downward going. However, the plot on the left has a clear slope and a high correlation coefficient $(\sim 1)$, and is clearly an upward going event. The muon tracks are then checked for directional consistency. The vertex direction cosine should be positive for upward going muons and is checked with the slope of the time vs. $y$ graph (number 12 from Table 6.1). 


\section{Track Fit - Strip Level}

As described in the "Track Fit" section of the track level cuts, the fit to the muon tracks is calculated by using Kalman filters in an iterative process through the track digits to measure the track's momentum, charge, and fit to the track's hits. The two left plots in Figure 6.4 shows the $\chi^{2} / n$.d.f. distributions. The plot on the bottom left shows the data and the plot on the top shows the MC distributions for events passing all the "track level" cuts as well as all the "strip level" cuts calculated from the standard reconstruction software. The shape of the data and the MC distributions do not agree. This is because the software reconstruction version (R1.14) is an older version, and had some "bugs" in the code like the calculation of the track fit $\chi^{2}$. All the bugs have been subsequently fixed in newer versions of the reconstruction software.

A new fit $\chi^{2}$ for the tracks is calculated to correct for this difference by comparing the reconstructed track position and the strip transverse position using the following equation:

$$
\chi_{n e w-f i t}^{2} / n d f=\frac{\frac{12}{w^{2}} \sum(t p o s-r p o s)^{2}}{n}
$$

where $n$ is the total number of track hits, the sum is over all $n, w$ is the width of the strips $(4.1 \mathrm{~cm})$, tpos are the transverse positions of the strips, and rpos are the reconstructed positions of the tracks. The two plots on the right in Figure 6.4 shows the new distributions, with Monte Carlo on the top and data on the bottom. The shapes of the recalculated fits for the Monte Carlo and the data agree much better than the Standard 

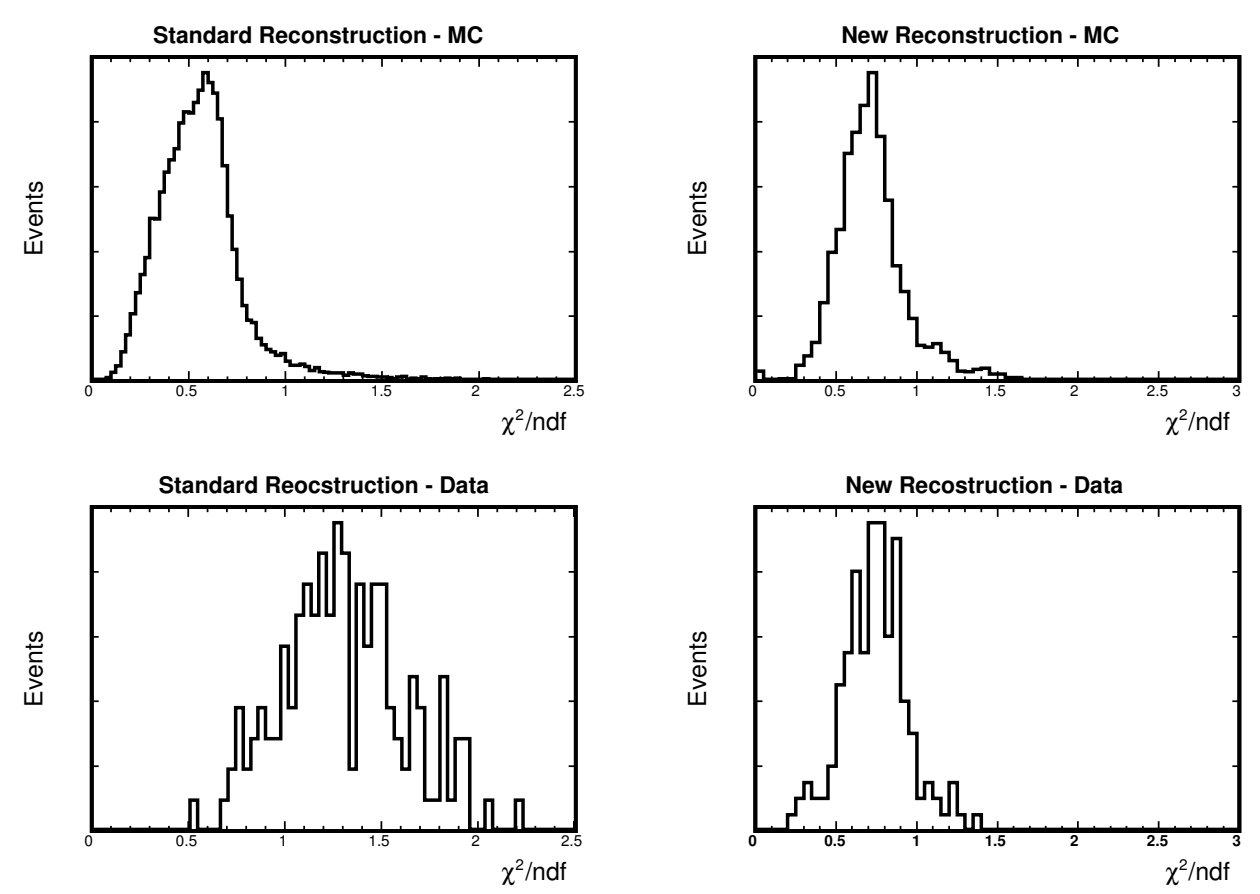

Figure 6.4: Fit $\chi^{2} / n d f$ for the tracks from Standard Reconstruction on the left and recalculated fit $\chi^{2} / n d f$ on the right.

Reconstruction distributions. Tracks with $\chi_{f i t}^{2} / n d f \leq 1.5$ are kept for the physics analysis (cut 13 from Table 6.1).

\section{Beam Events}

A fraction of the neutrino induced muons events selected are due to the NuMI beam that originates at Fermilab near Chicago, Illinois, approximately $735 \mathrm{~km}$ away. These events have been created as a result of the beam neutrinos interacting outside the detector in the rock, and mimic atmospheric neutrino induced muons. Using GPS timing for the beam spill in the Near Detector and the vertex time for each event, the beam events are selected 
out. The difference between the beam spill time and event vertex time should be on the order of $50 \mu$ seconds. A conservative cut of a $70 \mu$ seconds window is applied to select the beam events. Figure 6.5 shows the selected neutrino induced muon events (without
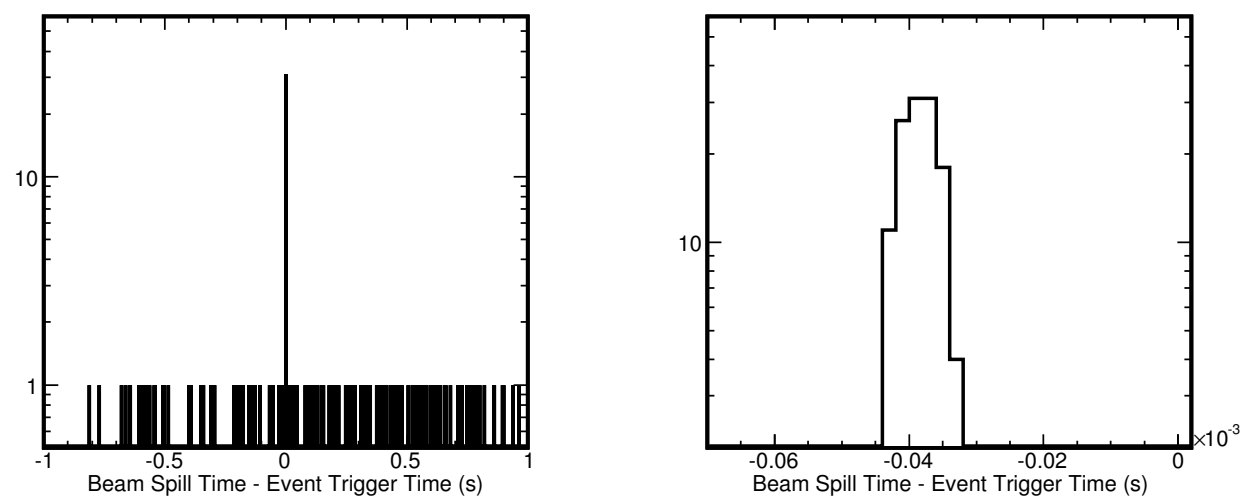

Figure 6.5: The selected horizontal and upward going events as a function of the difference between the beam spill time in the Near Detector at Fermilab near Chicago, Illinois, and the event trigger time at the Far Detector.

beam de-selection) as a function of the time difference between the beam spill time in the Near Detector and the event trigger time in the MINOS Far Detector. The plot on the left shows all selected neutrino induced events. The plot on the right shows the zoomed in version for the plot on the left around the large peak. These events fall within the 70 $\mu$ seconds window allowed by the beam selection cut. 


\section{Selection Summary}

As a result of applying the selection cuts described so far, a total of 17.1 million muon events have been selected. These muon track events have reliable demultiplexing, and they are single muons. They have a long enough track length to be ruled out as background events such as pion tracks. The events also have reliable timing and fit to the track hits. Their timing in the $y$ direction is clear and their fit to the $y$ direction timing is good. This enables these events to be labeled as either upward going or downward going muons. Since the vertex direction in $y$ checked with the slope of the timing in $y$, the vertex direction in $y$ is also an indicator of the events' up/down directionality. A vertex direction cut can now be made to select the final neutrino induced muon candidate sample.

\subsection{Neutrino Induced Candidate Events}

\subsubsection{Upward Going Events}

The neutrino induced upward going muons are created as a result of neutrino interaction in the rock slightly below the detector. The signature for these events is the positive slope in the track's timing in the $y$ direction. The neutrinos interacting in the rock travel a distance ranging from several hundred kilometers up to approximately thirteen thousand kilometers. The upward going muons coming from near the horizon travel over five hundred kilometers. The cosmic ray background in this region is negligible since the

intensity falls off as $E^{-2.7}$, where $E$ is the cosmic ray muon energy. Only the highest energy 
cosmic rays can survive the trip through the rock overburden of the MINOS Far Detector.

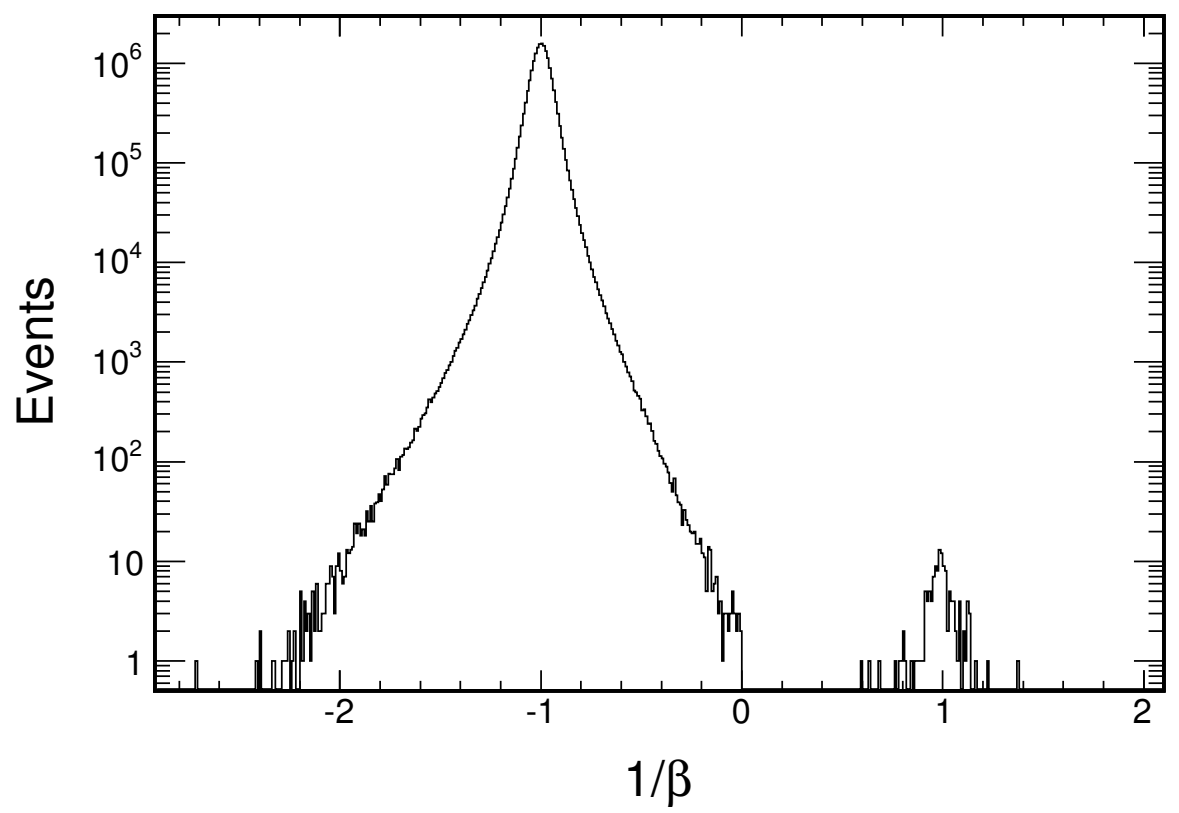

Figure 6.6: Inverse $\beta$ distribution for all muons passing the strip level selection cuts.

Thus these upward going muon events coming from near the horizon are neutrino induced muons.

A total of 135 candidate events out of the 17.1 million events passing the strip level cuts are upward going muons. Figure 6.6] shows inverse $\beta$ distribution for all the data events passing the strip level cuts. The upward going muons have a positive $1 / \beta$ value, while the downward going muons have a negative $1 / \beta$ value. The distribution is very well separated and distinguishes the up/down directionality of the muons. The background in the positive $1 / \beta$ region consists primarily of muons from neutrino interactions inside the 
detector, the estimation of which will be discussed in Section 6.4. Among the downward going muons, the events with a high negative value of $1 / \beta(\leq-1.8)$ have poor timing calibrations. Although the events' timing in the $y$ direction clearly indicates that the events are downward going, poor timing calibration causes a spread in the timing and results in higher $|1 / \beta|$ values. On the other hand, the downward going events with lower values of $|1 / \beta|(\leq-0.2)$ have a lower number of track timing digits causing the timing distribution in the $y$ direction to be random, and making the slope of $d t / d s$ small. The downward going muon sample has a mean of -1.0 in $1 / \beta$ with an r.m.s. of 0.07 , while the upward going muon sample has a mean of +1.0 in $1 / \beta$ with an r.m.s. of $0.09 \pm 0.01$, which is fairly compatible with the downward going r.m.s.

Although the inverse beta distribution of the upward going sample is well separated from that of the downward going sample, there are several upwards going events with $1 / \beta$ values in the $0.6-0.7$ region, and $1 / \beta>1.2$ region. Figure 6.7 shows one such example of the events. This event's measured value of $1 / \beta$ is 0.6 . The two plots of the left panel are the $x$ and the $y$ positions as a function of the $z$ values. The solid circles are the track hits as identified by the standard reconstruction. The open circle data point are due to shower hits. The top plot on the middle panel is the reconstructed $y$ values as a function of the reconstructed $x$ values. The dashed circle in this view is the 3.5 meters standard fiducial radius set to identify contained events. The two plots on the bottom of the middle panel show the time vs $y$ and the time vs $z$ positions. In both these views there are two timing data points that are seen as outliers. They have most likely been caused by cross 

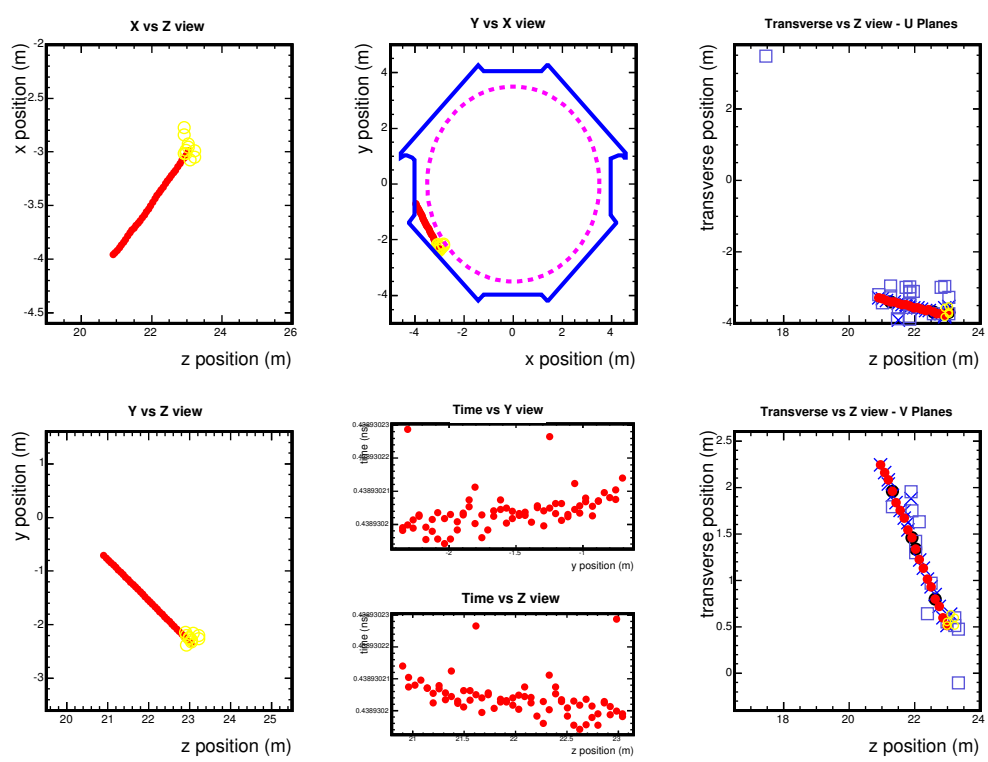

Figure 6.7: Upward going candidate event from Run 32868, Snarl 150167. The two plots on the left panel are $x$ and $y$ data points as a function of the $z$ positions. The $y$ vs. $x$ view is on the top of the center panel. The solid line in this plot depicts the outer shape of the detector, and the dashed line of the $3.5 \mathrm{~m}$ fiducial radius. Timing in the $y$ direction is plotted in the middle of the center panel, and timing in the $z$ direction is on the bottom. The panel on the right shows the transverse $\mathrm{U}$ and $\mathrm{V}$ positions (see Chapter 4 for description) as a function of the $z$ positions. The red points refer to the track, and the yellow (open circle) points refer to the shower as identified by the reconstruction. The open squares are mostly due to cross-talks between the photo-multiplier tube pixels.

talk that the reconstruction mis-identified as part of the track. These two timing hits are also responsible for a poor fit to the timing, making the $1 / \beta$ value of the event to be as low as 0.6. If the two outliers are ignored, the slope appears more positive and the fit to the slope significantly increases. Thus the event is kept in the sample for the analysis. Since the timing in the $y$ direction has a positive slope, this event is seen to be entering from the bottom of the detector as depicted in the "Y vs X view" plot. Similarly, looking 
at the slope of the timing in the $z$ direction (negative), the $z$ vertex of the detector is at $z=23$ meters.

More examples of the upward going candidate events can be found at the end of this chapter. A complete list of the neutrino induced events can be found in Appendix \$, The neutrinos producing the upward going muons travel a wide range of distances, and since the probability of oscillation depends on the distance traveled, the muons with higher zenith angle (larger baseline) will show greater oscillations. A combined energy and zenith angle oscillation fit in the analysis chapter will show that the effect of oscillation is greatest for lower energy muons in the high zenith angle range.

\subsubsection{Horizontal Events}

Horizontal muons are "shallow" events that are created as a result of neutrino interaction in the rock surrounding the detector where the muons appear to be downward going. These events are identified by a negative slope of the timing in the $y$ direction, and their vertex direction cosine is less than zero. The atmospheric neutrinos producing these muons travel a baseline distance from 200 to 500 kilometers of earth matter. There are virtually no cosmic ray muons that can survive traveling to the detector in this baseline range. Typically the energy for these muons surviving the rock overburden of the detector is in the order of $E_{\mu}>100 \mathrm{TeV}$. Since the primary cosmic ray energy spectrum falls off very steeply $\left(E^{-2.7}\right)$, the background due to cosmic ray for these horizontal muons is relatively small. 


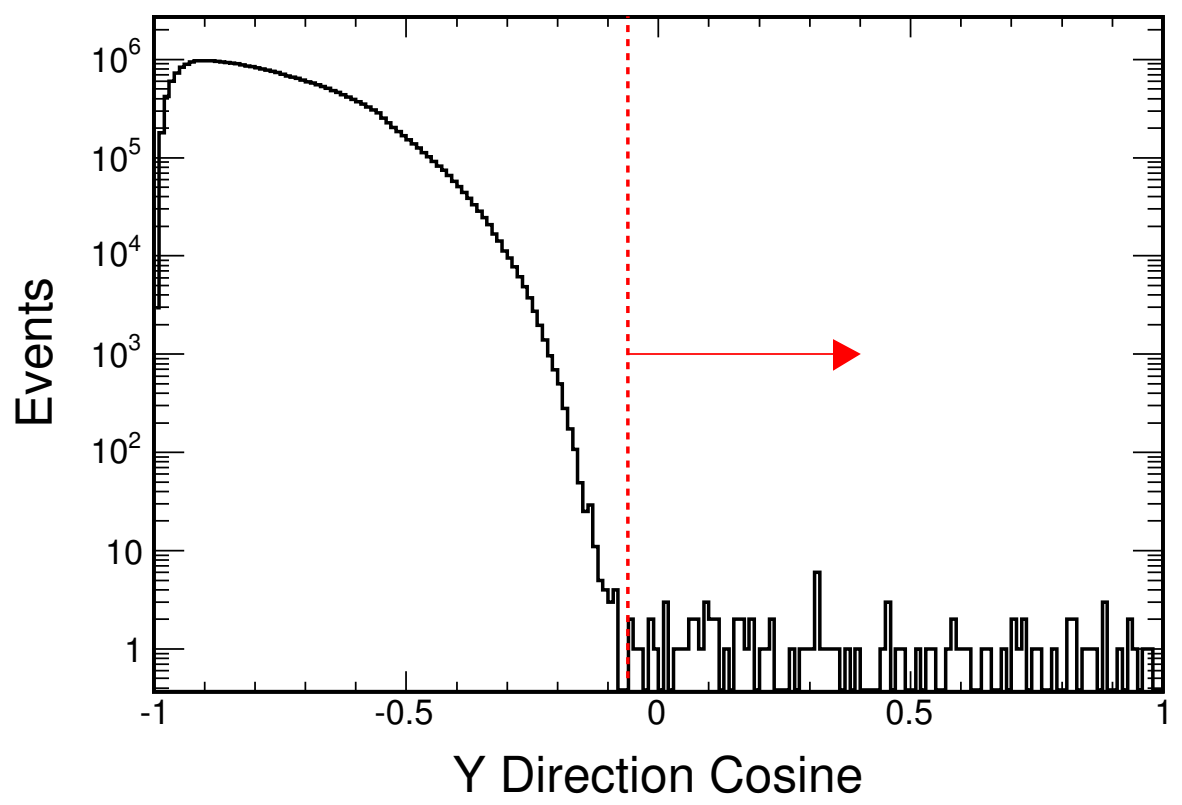

Figure 6.8: The vertex $y$ direction cosine for data passing the strip level cuts. The final cut for identifying neutrino induced muons is illustrated by the vertical dashed line and the arrow.

Figure 6.8 shows the $y$ direction cosine (negative $\cos \left[\theta_{\text {zenith }}\right]$ ) for all the data events. The dashed vertical line represents the cut imposed for the neutrino induced muons. The choice of the vertex $y$ direction cosine $(d \cos y)$ cut at -0.06 is chosen to minimize the cosmic ray background, and will be explained in Section 6.4. There are 13 events in the region $-0.06<d \operatorname{cosy}<0.0$, that are identified as horizontal events. Figure 6.9 shows an example of a horizontal neutrino induced muon candidate. Since the slope of the timing in the $z$ direction is negative, the muon track enters the first Super Module (SM) at $z=0$. It crosses the entire first SM and part of the second SM. The first and the second Super 

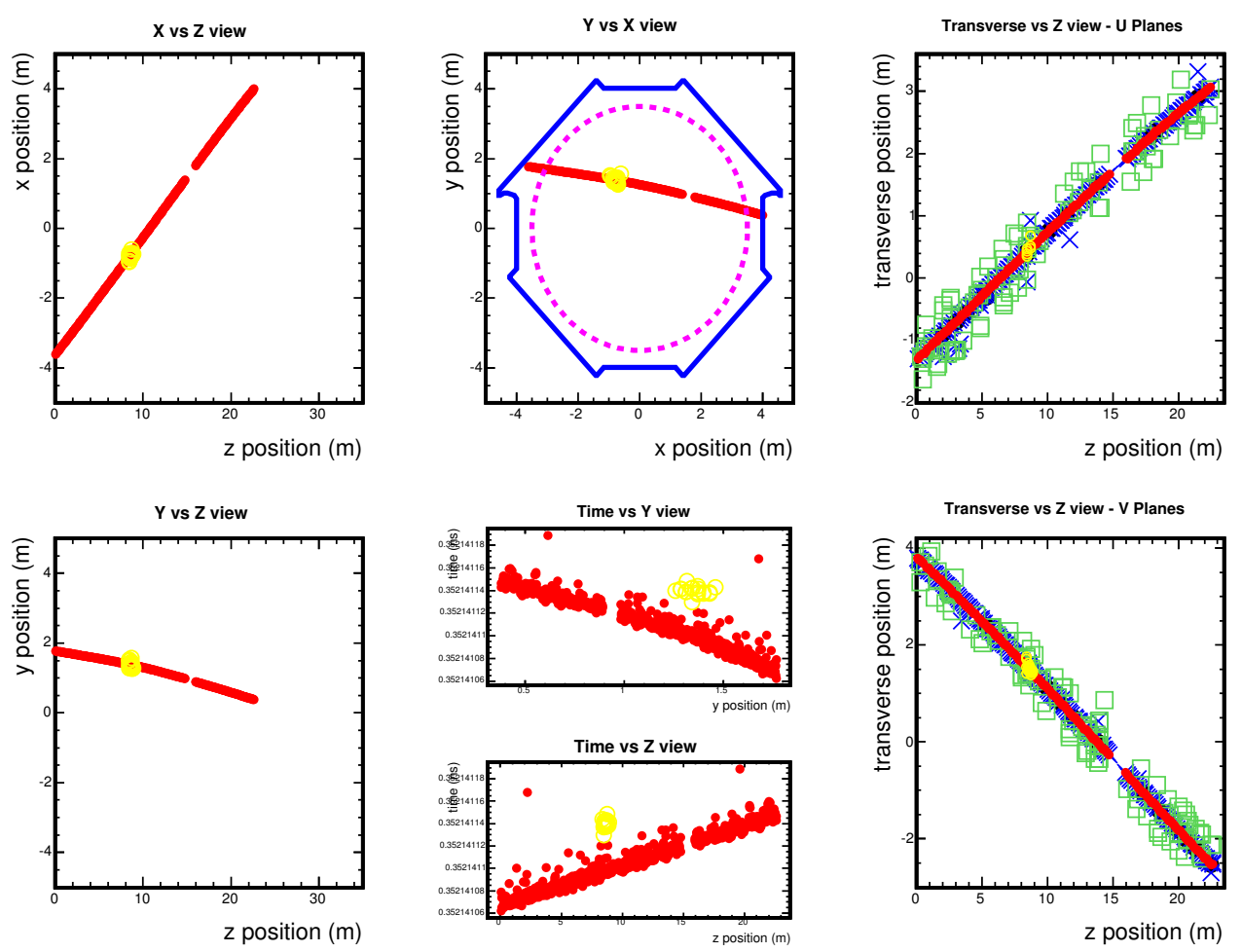

Figure 6.9: Example of a neutrino induced horizontal muon candidate event (Run 18900, Snarl 94973). See the caption in the Figure 6.7 for an explanation of the event display. The timing in the $y$ direction indicates that this is a downward going event. The gap seen in the plots appears because the muon crosses the gap between the two Super Modules.

Modules are physically separated by a distance of one meter, and this is seen as a gap in all the plots.

Horizontal muons play an important role in the overall flux normalization. Neutrinos that interact in the rock to produce these events travel a distance of several hundred kilometers and have similar energies to the upward going muons traveling thousands of kilometers. Therefore, in general, the neutrinos that produce these events will have a lower 
value of $L / E$ compared to those in the upward going category. Thus these muons have a higher survival probability, and can be an important check for the flux normalization.

\subsubsection{Charge/Momentum Selection}

MINOS is the first underground detector of it's kind to be able to distinguish positive muons from negative ones. The MINOS detector is also able to measure the momentum from the curvature of the tracks due to the relatively high magnetic field. It is important to separate the charge of the muons in order to test CPT conservation.

In order to measure a charge ratio, it is essential to obtain a relatively clean sample

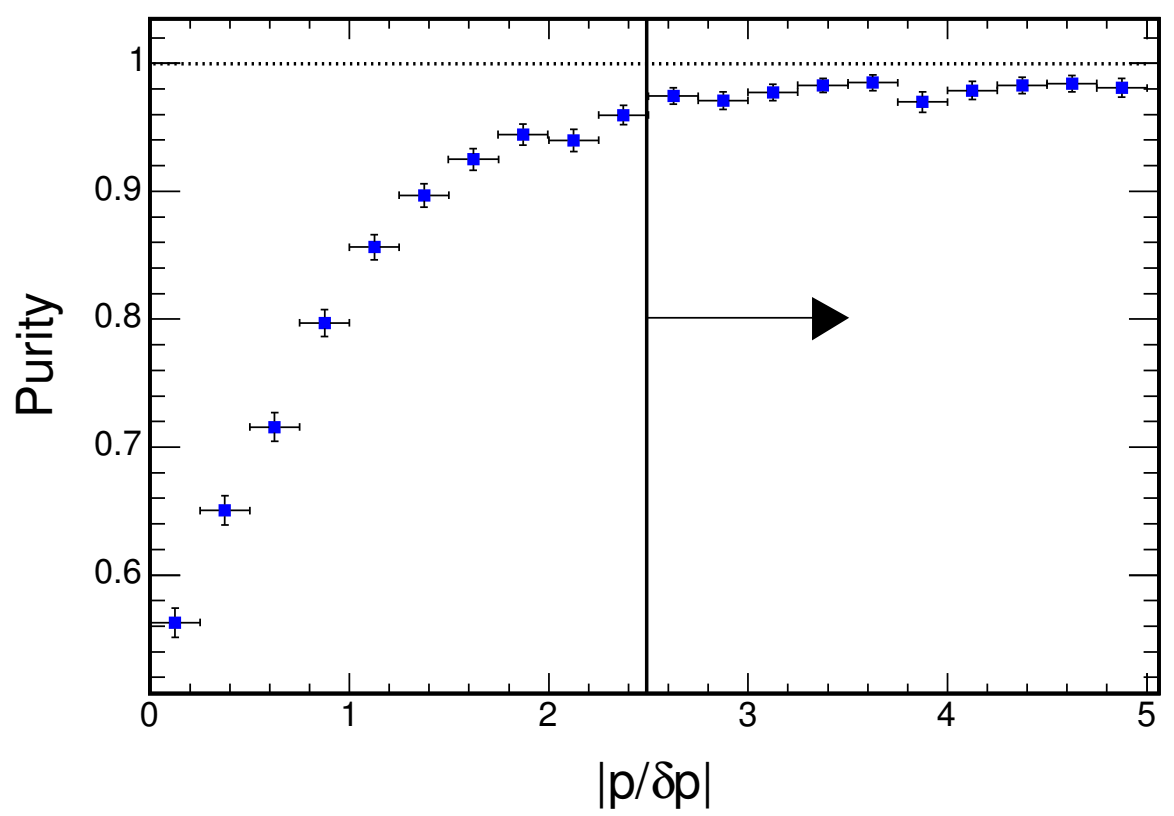

Figure 6.10: Charge/momentum purity as a function of measured momenta divided by the measured error. 
of positive and negative muons. This is done by looking at the binned purity distribution for all muon momenta in the neutrino induced Monte Carlo sample as shown in Figure 6.10. The purity is plotted as a function of the measured momenta over error in the measured momenta $(|p / \delta p|)$, where $p$ is the measured momentum. The binned purity is defined as the ratio of the number of reconstructed muons with the correct charge to the total number of muons for a given bin in $|p / \delta p|$. Higher bins in $|p / \delta p|$ give a higher fraction of reconstructed muons with a correctly identified charge. A cut is imposed at $|p / \delta p| \geq 2.5$. Extrapolating the average purity at the cut of 2.5 gives a purity of $\sim 97 \%$, or an impurity of $\sim 3 \%$. A cut at $|p / \delta p| \geq 2.5$ is sufficient to assume the sample has muons with well measured momenta and identified charge. The sample of events is called high charge/momentum resolution events or "high resolution" events.

\subsubsection{Event Distributions Summary}

Out of a total of 148 candidate events, 45 have measured momenta less than $12 \mathrm{GeV}$, and 103 events have measured momenta $12 \mathrm{GeV}$ or greater. The choice of $12 \mathrm{GeV}$ as the low momentum selection will be discussed in Chapter 7 These events include both high and low charge/momentum resolution events. In the high resolution sample, 42 events have measured momenta less than $12 \mathrm{GeV}$, and 40 events have measured momenta greater than $12 \mathrm{GeV}$. Also in the high resolution sample there are $30 \mu^{-}$and $52 \mu^{+}$candidates. Table 6.2 summarizes the distributions of the candidate events. 
Table 6.2: Distribution of the selected candidate events

\begin{tabular}{|l|c|c|}
\hline & $p \leq 12 \mathrm{GeV}$ & $p>12 \mathrm{GeV}$ \\
\hline All Candidate Events & 45 & 103 \\
\hline$\mu^{-}$(High Resolution Events) & 26 & 26 \\
\hline$\mu^{+}$(High Resolution Events) & 16 & 14 \\
\hline
\end{tabular}

\subsection{Background}

There are two sources of background considered in this analysis. The first source is the cosmic ray muons coming from slightly above the horizon. This is estimated by examining the behavior of the data in the vertex $y$ direction cosine distribution of the muon tracks, and extrapolating a fit to the region of interest. A second source from contained vertex events is estimated by examining the contained atmospheric neutrino Monte Carlo sample. The background from misidentified downward going cosmic ray muons shown in Figure 6.6 is negligible.

\subsubsection{Cosmic Ray Muons}

To estimate the cosmic ray background near the horizon, the distribution of the $y$ direction cosine $(d \cos y)$ is closely examined. Figure 6.8 shows the $d \cos y$ distribution for the whole data sample. The shape of the distribution near and slightly above the the horizon $(-0.2$ $<d \cos y<-0.1)$ is steeply falling. Hence an exponential fit is performed in the region of $-0.2<d \cos y<-0.1$ to predict the number of cosmic ray muons in the region slightly above the horizon $(-0.1<d \cos y<0)$. The left panel of the Figure 6.11 shows a zoomed in 

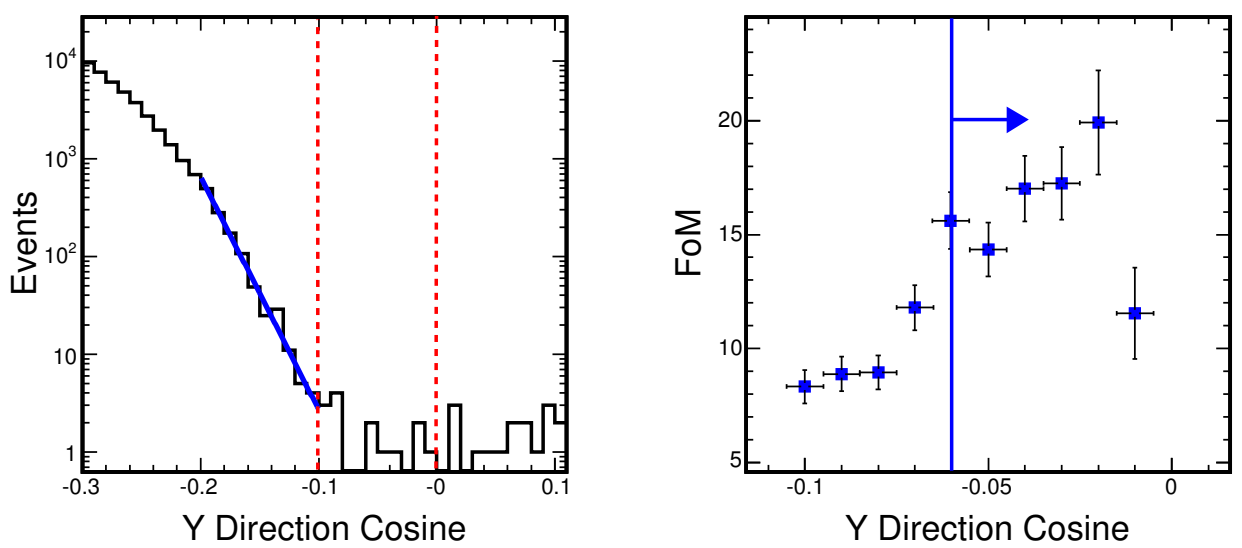

Figure 6.11: The left panel shows a zoomed-in distribution of the vertex $y$ direction cosine $(d \cos y)$ plot of Figure 6.8. The solid line is the exponential fit performed. The dashed lines cover the region of $-0.1<d \cos y<0$, where the background is estimated. The panel on the right shows the figure of merit (FoM) calculated for the horizontal region, and the cut for selecting the neutrino induced muon candidates.

version of Figure 6.8. The exponential fit has the form $y=e^{(c+m x)}$, where $c=-4.5 \pm 0.3$ and $m=-54.9 \pm 1.7$, and the $\chi^{2} / n . d . f .=8.6 / 8$. This is a decent fit and can be used to estimate the background in the region of $-0.1<d \cos y<0$. The estimated background is 2.7 events at $d \cos y=-0.1$. This is comparable to the number of data events (2) in that $d \cos y$ bin. On the other hand, the background is 0.01 at $d \cos y=0$, a very small contribution. The cosmic ray background for upward going muons $(d \cos y>0)$ is even smaller, and is ignored for this analysis.

There are a total of 21 candidate events in $-0.1<d \cos y<0$, and a total background of 6.4 events in the same range. To estimate the best $d \cos y$ cut, where the background is relatively low and the loss of data is minimal, a figure of merit (FoM) is calculated for 
different values of $d \cos y$ using, FoM $=$ Data $/ \sqrt{B G}$, where $B G$ is the integrated background estimated from the fit to $d \cos y$, and Data is the total number of candidate events in that range. The panel on the right on the Figure 6.11 shows the FoM in the horizontal region. The FoM values increase to a maximum for the $d \cos y$ value around -0.03 , and drops to zero at $d \cos y$ at 0 . Ideally, the choice for placing the $d \cos y$ cut would be around the maximum value for the FoM. However, a looser cut of $d \cos y$ at -0.06 is placed to allow more data events for the analysis. The estimated background for $-0.06<d \cos y<0$ is 0.7 events, and the number of data events is 13 .

\subsubsection{Contained Upward Going Muons}

Another source of background is the upward going muons created as a result of neutrino interaction inside the detector but outside the fiducial volume cut described in Section 6.2 These events are not simulated in the neutrino induced MC sample which only includes interactions in the rock surrounding the detector. To estimate the background from this source, a sample of the contained atmospheric neutrino Monte Carlo is analyzed. Figure 6.12 shows the square of the radial distance from the center of the detector for the events in the MC sample that pass the selection cuts described in Section 6.2 except for the containment cut (9 from Table 6.11). The vertical line is placed at the square of the radial containment cut. The total number of oscillated events outside the containment cut (3.7 meters) is $14.8 \%$ of the total number of the contained events (left of vertical line). The radial vertex distribution falls off rapidly near the outer edge of the detector (vertex 


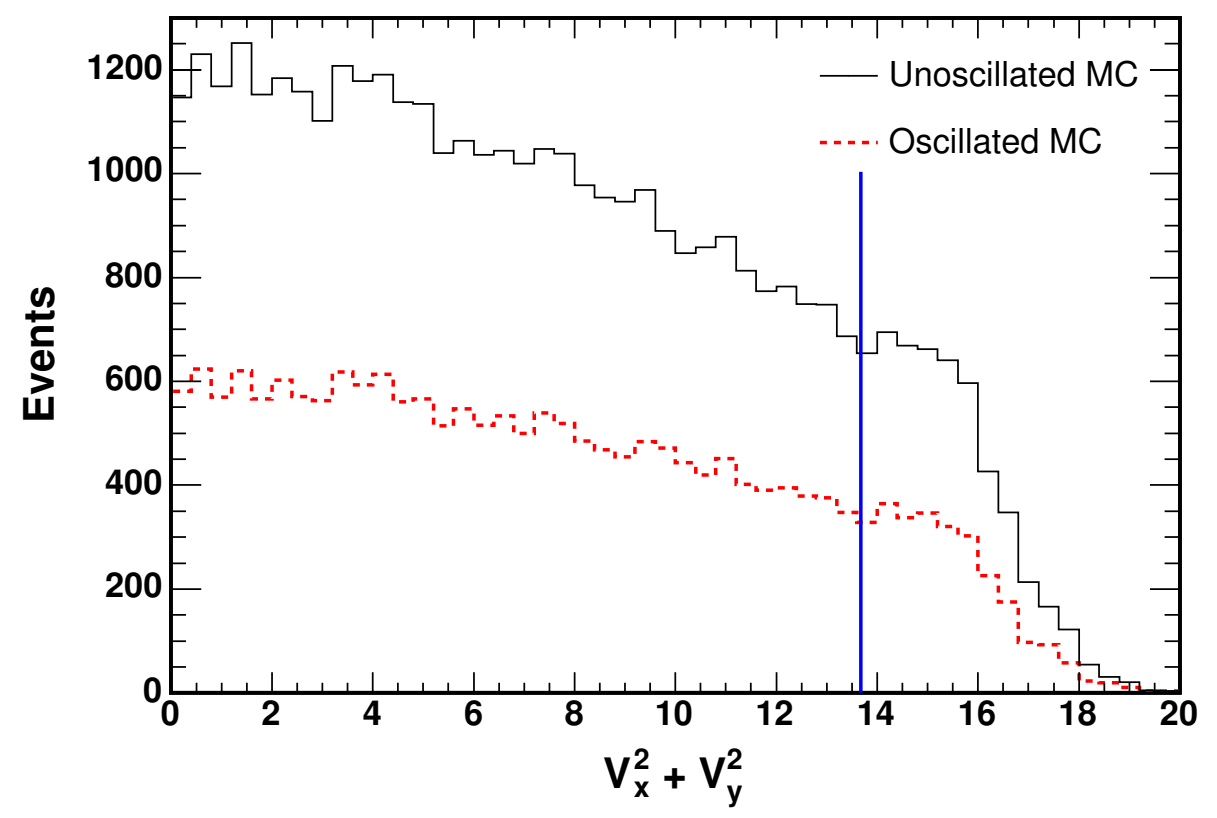

Figure 6.12: Square of the radial vertex distance from the center of the detector for contained oscillated and unoscillated Monte Carlo sample. The vertical line is placed at the contained radius cut $(3.7 \mathrm{~m})$

radius $>4 \mathrm{~m}$ ). This is because the events near the edge of the detector have a smaller probability of being reconstructed. Figure 6.13 shows the square of the radial distance from the center of the detector for all data events. There are 24 data events that lie within the contained radius cut and pass all the other cuts. Scaling this to the contained Monte Carlo sample gives an estimated background of 3.56 events outside the contained radius cut. Adding this to the cosmic ray muon background gives a total background of 4.26 events. The background will contribute to the low to high momentum ratio calculated for the oscillation analysis in Chapter 7 


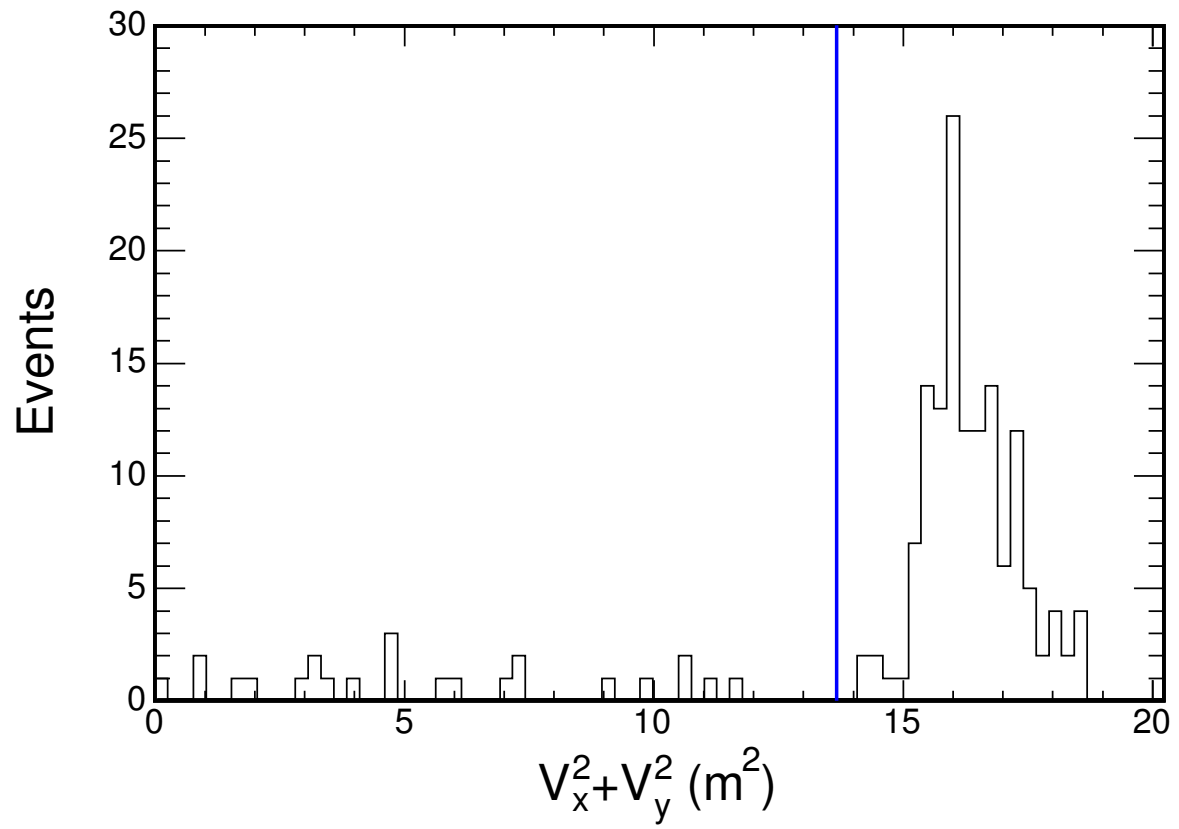

Figure 6.13: Square of the radial vertex distance from the center of the detector for data. The vertical line is placed at the contained radius cut $(3.7 \mathrm{~m})$.

\subsection{Systematic Uncertainties}

The systematic uncertainties considered here come from event selections only. Uncertainties due to physics modeling are discussed in Section 7.2. The errors in event selection do not contribute significantly to the final ratios calculated in Chapter 7 . Only the major contributers are described here. The systematic uncertainties due to the selection cuts are obtained by first determining the $1 \sigma$ value of the fractional uncertainty for each cut. The percentage difference between selecting the data and the neutrino induced Monte Carlo provides a reasonable value for $1 \sigma$ of the fractional uncertainty, since the cuts affect the 
data and the Monte Carlo differently.

As seen in Table 6.1, the relative efficiencies of the data and the Monte Carlo sample are incomparable. This is because of the lack of multiple muons in the simulations makes a large difference in the relative efficiency. Thus the efficiencies are normalized at the "Number of Tracks" cut (2 from Table 6.1) to account for the difference. Table 6.3 shows the new normalized efficiency of the selection cuts starting from the "Number of Tracks" cut.

Table 6.3: Normalized selection efficiency

\begin{tabular}{|l|c|c|c|}
\hline \hline & Data & Cosmic MC & Rock Muon MC \\
\hline \hline 2. Number of Tracks & 0.78 & 0.79 & 0.79 \\
\hline 3. Number of Planes & 0.58 & 0.59 & 0.62 \\
\hline 4. Track Length & 0.58 & 0.59 & 0.61 \\
\hline 5. Track Time Digits & 0.55 & 0.57 & 0.59 \\
\hline 6. $\chi_{\text {time }}^{2} / n d f$ & 0.55 & 0.57 & 0.57 \\
\hline 7. $\chi_{\text {fit }}^{2} / n d f$ & 0.55 & 0.57 & 0.57 \\
\hline 8. Vertex Y Reconstruction & 0.54 & 0.55 & 0.57 \\
\hline 9. Contained Vertex & 0.52 & 0.54 & 0.55 \\
\hline 10. East \& West Side Timing & 0.52 & 0.54 & 0.54 \\
\hline 11. Time vs. Y Correlation & 0.52 & 0.54 & 0.54 \\
\hline 12. Directional Consistency & 0.51 & 0.53 & 0.53 \\
\hline 13. $\chi_{\text {new }- \text { fit }}^{2} / n d f$ & 0.49 & 0.52 & 0.52 \\
\hline \hline
\end{tabular}

The first two sources of systematic uncertainty are the $\chi_{\text {fit }}^{2} / n d f$ and $\chi_{n e w-f i t}^{2} / n d f$ cuts. Comparing the normalized efficiency of the selection cuts in Table 6.3 the difference in efficiency is $\pm 2.0 \%$ and $\pm 3.0 \%$. For these two selection cuts, a more conservative fractional error of $\pm 4.0 \%$ is assumed. The reason for doing so is that even with a conservative approach, the final uncertainties calculated in Chapter 7 are very small. The systematic 
uncertainty due to the selection cuts is, in general, smaller compared to the uncertainty for the physics modeling, and even smaller compared to the statistical uncertainties for the low to high momentum ratio calculated in the analysis chapter.

The difference in the normalized efficiency for "Time vs. Y Correlation" is $\pm 2.0 \%$, and a conservative fractional uncertainty of $\pm 3.0 \%$ is assigned to this cut. For the "Track Time Digits" cut (5) in Table 6.3 a similar conservative $\pm 5.0 \%$ fractional uncertainty is assigned. Two additional sources are included with the charge/momentum selection cut described in Section 6.3.3 A fractional error of $\pm 3.0 \%$ is assigned in purity of this selection cut. The $|p / \delta p|$ cut removes $80 \%$ the data of the events passing the strip level cuts, and similarly removes $77 \%$ of the simulated events. This is a difference of $3.0 \%$, and a fractional error of $\pm 4.0 \%$ is assigned to this cut in order to be conservative. Table 6.4 shows the $1 \sigma$ fractional uncertainty due to the selection cuts for the sources that have any significant contribution to the final ratio discussed in Section 7.2

Table 6.4: $1 \sigma$ fraction systematic uncertainty due to selection cuts

\begin{tabular}{|c|c|}
\hline Source & Fractional Error \\
\hline \hline$\chi_{\text {fit }}^{2} / n d f$ & $4.0 \%$ \\
\hline$\chi_{\text {new-fit }}^{2} / n d f$ & $4.0 \%$ \\
\hline Time vs. Y correlation & $3.0 \%$ \\
\hline Track Time Digits & $5.0 \%$ \\
\hline Charge Purity & $3.0 \%$ \\
\hline$|p / \delta p|$ & $4.0 \%$ \\
\hline
\end{tabular}



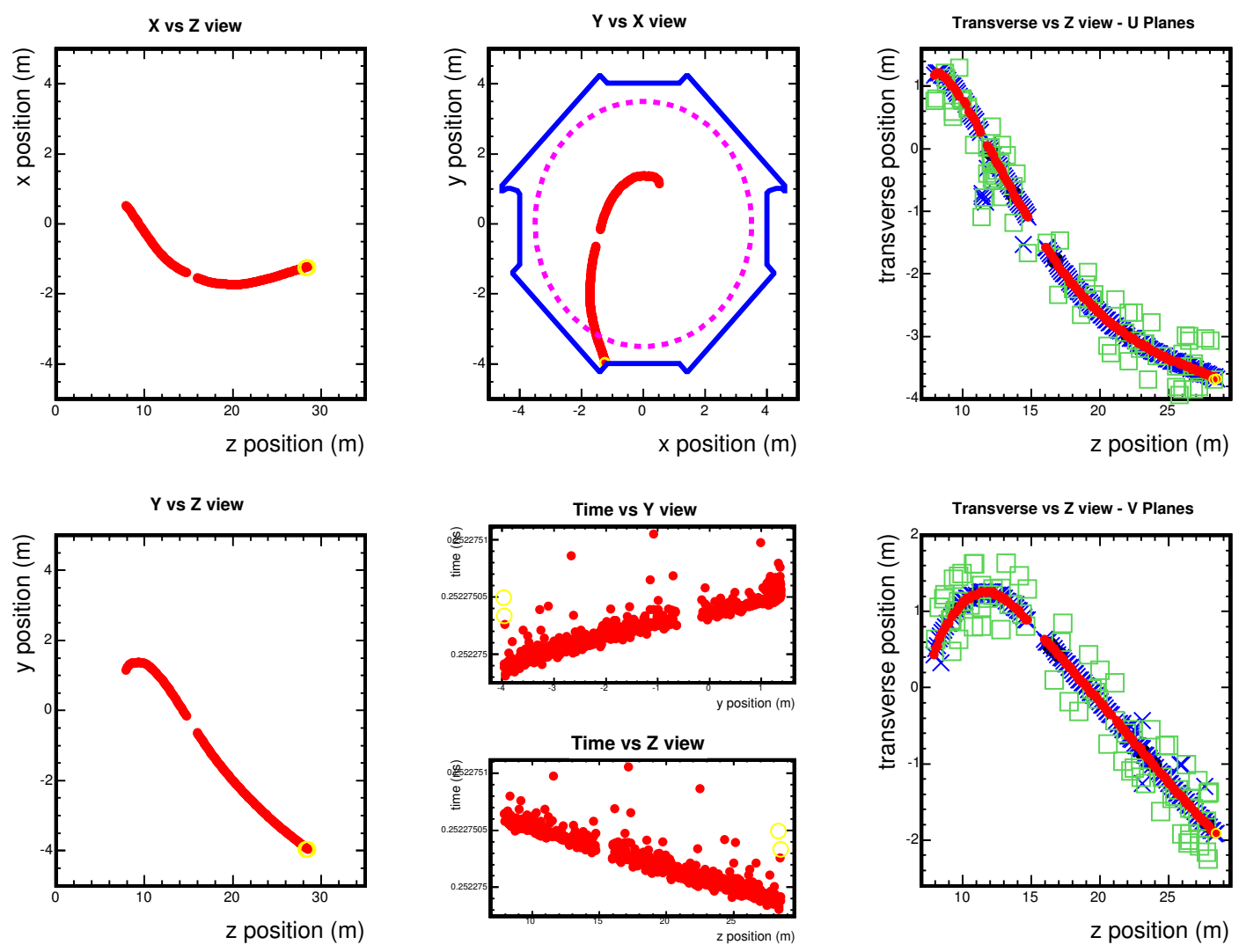

Figure 6.14: Upward going candidate event (Run 29449, Snarl 92140). The measured momentum from the curvature is $13 \mathrm{GeV}$. Since the $|p / \delta p|$ value is 72 , this events falls in the high resolution sample. See the caption for the Figure 6.7 for an explanation of the event display. 

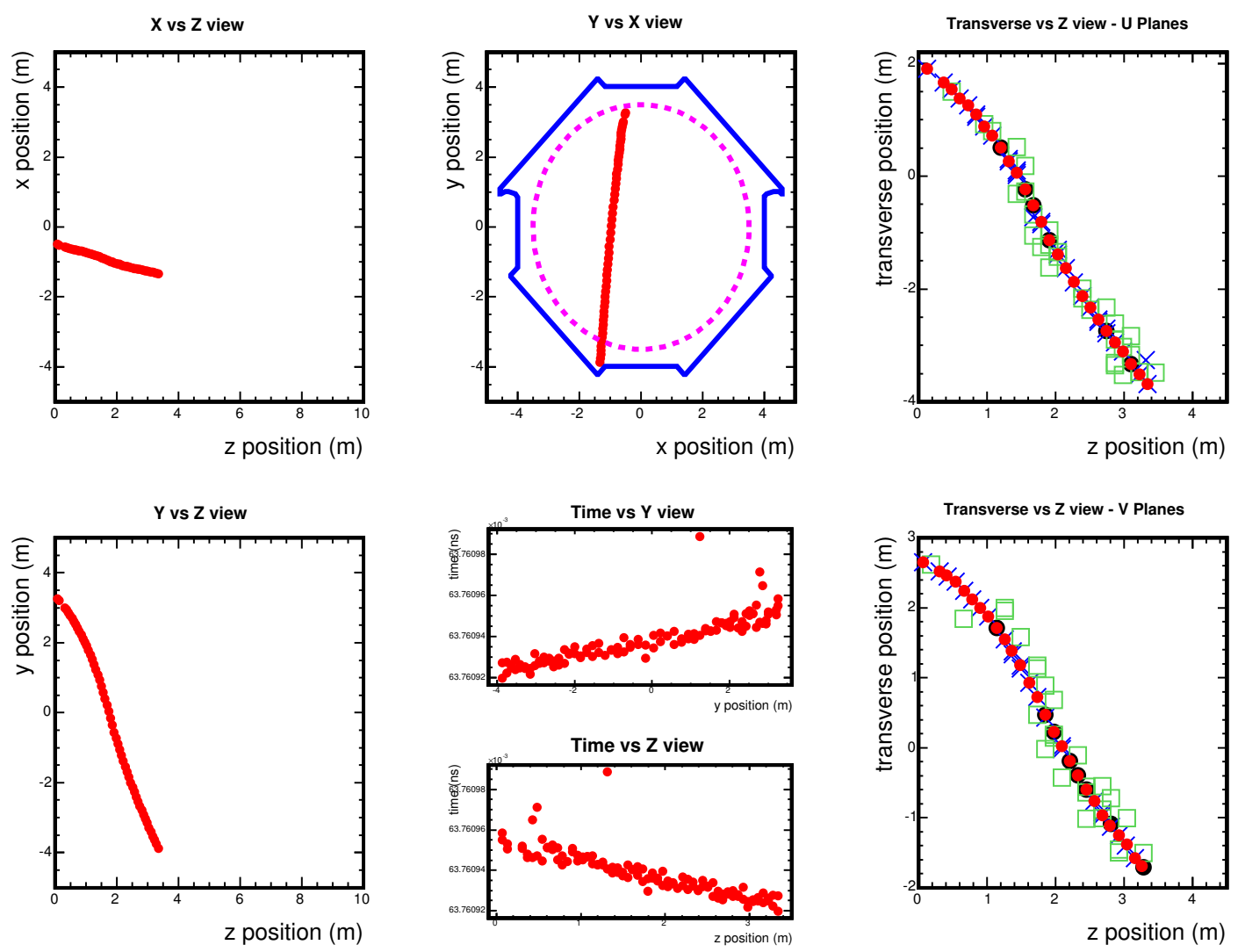

Figure 6.15: Candidate data event (Run 19292, Snarl 49901). This event has a clear positive slope in the "Time vs. Y View". The measured momentum from curvature is well resolved since $|p / \delta p|=21$. See the caption for the Figure 6.7 for an explanation of the event display. 

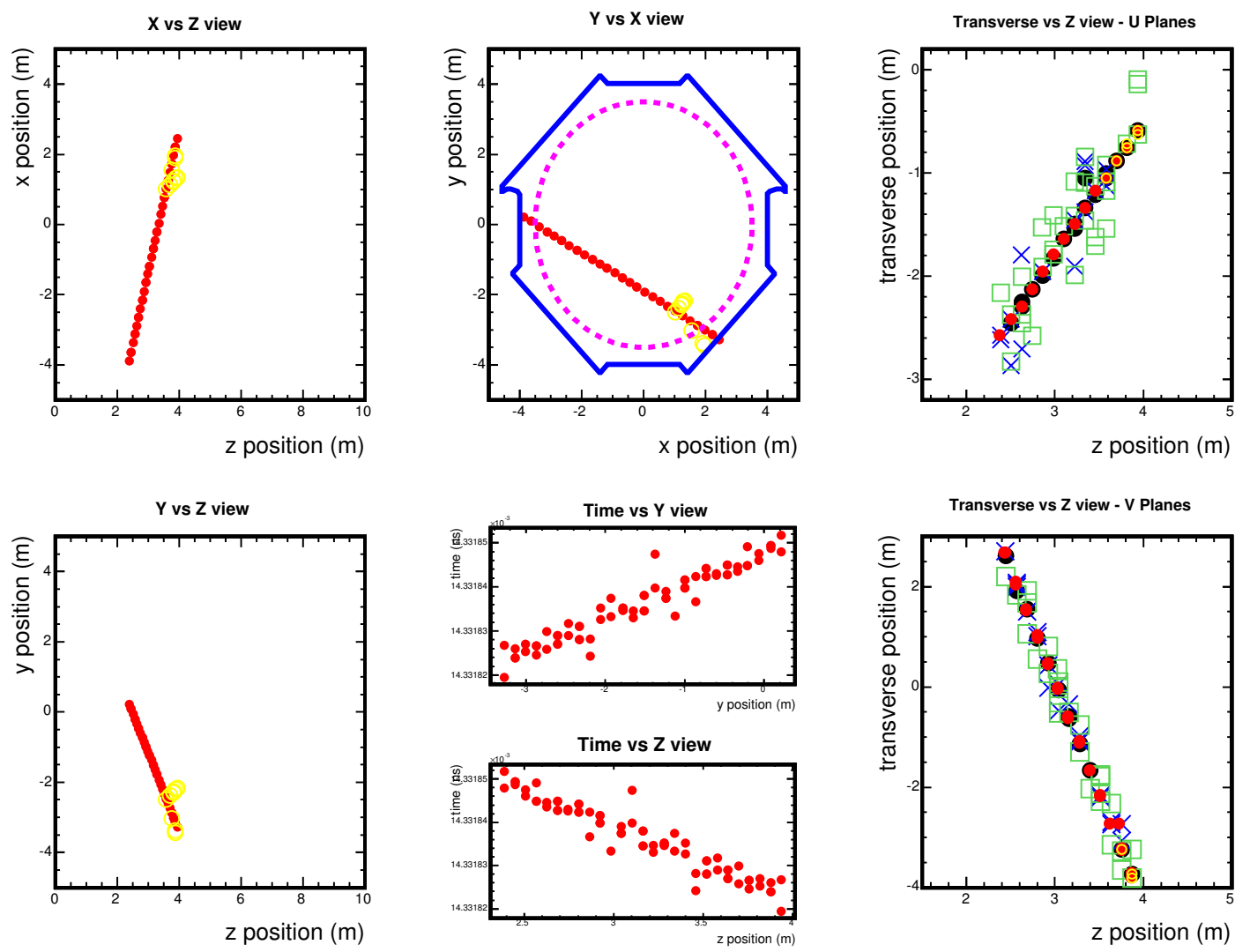

Figure 6.16: Candidate data event (Run 28267, Snarl 56432). This event has a positive slope in the "Time vs. Y View". The measured momentum from the curvature not is well resolved since $|p / \delta p| \approx 0$ and is a high energy event. However, this upward going muon can be used in the combined energy and zenith angle oscillation analysis. See the caption for the Figure 6.7 for an explanation of the event display. 


\section{Chapter 7}

\section{Oscillation Analysis}

\subsection{Introduction}

The goal of this chapter is to compare the measured and predicted distributions of the neutrino induced muons to test for the dominant atmospheric $\nu_{\mu} \rightarrow \nu_{\tau}$ mode oscillations as well as search for CPT violation. The data and the Monte Carlo are divided into two samples. The first sample includes all muon events, and the second includes muons with well measured momenta. Each sample is further divided into two momentum bins. Events with momenta less than $12 \mathrm{GeV}$ are called "low momentum events", and similarly, events with momenta greater than $12 \mathrm{GeV}$ are called "high momentum events". The low to high muon momentum ratio for data is compared with that of the Monte Carlo sample. To measure the oscillation parameters, a maximum log-likelihood analysis is performed with normalization systematic uncertainty. Finally, the oscillation sensitivity for the data 
exposure is calculated and a 10 year prediction is determined using the best fit results obtained from the data to Monte Carlo (MC) oscillation analysis.
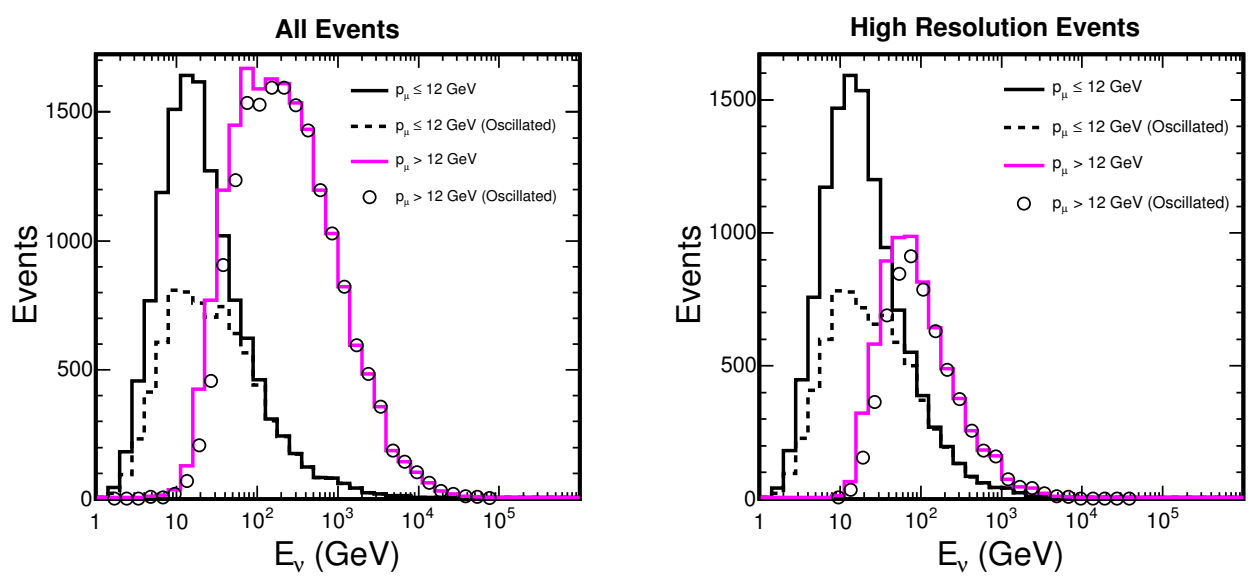

Figure 7.1: Parent neutrino energy distribution from the MC sample for the all data sample and the high resolution sample. The oscillation parameters are taken from the Super-Kamiokande publication [18].

\subsection{Low to High Momentum Ratio}

One method of looking for neutrino oscillations is to calculate the ratio of muons from lower energy neutrinos, which are generally more likely to show oscillation, to that of muons from the higher energy neutrinos, and compare that ratio to the unoscillated Monte Carlo. The data and the neutrino induced Monte Carlo are first divided into two samples. Events passing all the selection cuts in Section 6.2 are put into the "all events" sample, and the muons passing momentum resolution cut $(|p / \delta p| \geq 2.5)$ from Section 6.3 .3 in addition to the data selection cuts are put into the "high resolution" events sample. Note that the 
"high resolution" events are a subset of the events in the "all events" sample. Figure 7.1 shows the parent neutrino energy distribution for both of the event categories from the neutrino induced MC sample. The higher momentum muons, in general, originate from neutrinos of higher energies. Since the high energy neutrinos that produce high momentum muons do not oscillate significantly with the Super Kamiokande oscillation parameters, the oscillation effects are more dramatic for lower momentum muons. Therefore, the double ratio of the number of low to high momentum muons of the oscillated Monte Carlo to the unoscillated Monte Carlo should be less than unity if oscillations are present. To calculate the low to high momentum ratios for the data and the $\mathrm{MC}$, the optimum cut-off momentum is first determined.

\subsubsection{Low to High Momentum Boundary}

In order to determine the low to high momentum cut off, the ratio of ratios in the neutrino induced Monte Carlo sample, $R_{\text {low }}^{M C}$ high is calculated as follows,

$$
R_{\text {low } / \text { high }}^{M C}=\frac{\left(N_{\text {low }}^{\mu} / N_{\text {high }}^{\mu}\right)_{(\text {oscillated })}}{\left(N_{\text {low }}^{\mu} / N_{\text {high }}^{\mu}\right)_{(\text {unoscillated })}}
$$

where $\mathrm{N}$ is the total number of low/high momentum events. Figure 7.2 shows this ratio for the "all event" sample and "high resolution" samples. The values of $\Delta m^{2}=2.4 \times 10^{-3} \mathrm{eV}^{2}$ and $\sin ^{2}(2 \theta)=0.92$ are taken from the Super-Kamiokande best fit oscillation parameters [18. The ratios shown are fairly constant over the momentum cut off range. In order find the optimal cut off momentum, the significance of the statistical deviation of the ratios 

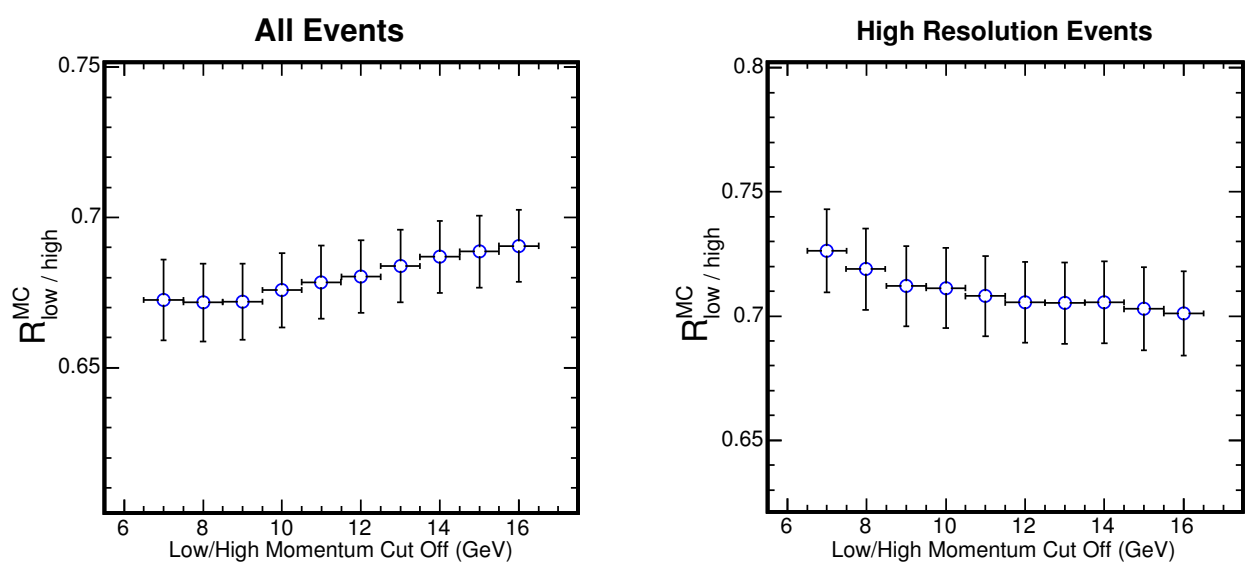

Figure 7.2: Ratio of the ratios of low to high momentum muons for the oscillated sample to that of the unoscillated sample for the neutrino induced Monte Carlo.

from unity is calculated and plotted in Figure 17.3. The highest statistical significance for both event samples is at $12 \mathrm{GeV}$ as shown in the figure. Events with momenta $12 \mathrm{GeV}$ or less are called "low momentum" events, and similarly the events with momenta greater than $12 \mathrm{GeV}$ are called "high momentum" events.

\subsubsection{Low to High Momentum Ratio - All Events}

A total of 148 candidate events that have passed the selection cuts described in Section 6.2 fall into the "all events" sample. The advantage of including low resolution events in this analysis is to gain higher statistics. However, the systematic uncertainty in the low to high momentum ratio is higher for these events and will be discussed in Section 7.3

As mentioned in Section [7.2 the "all events" sample contains both high resolution events and events with poor momentum measurement. Low momentum events are more 

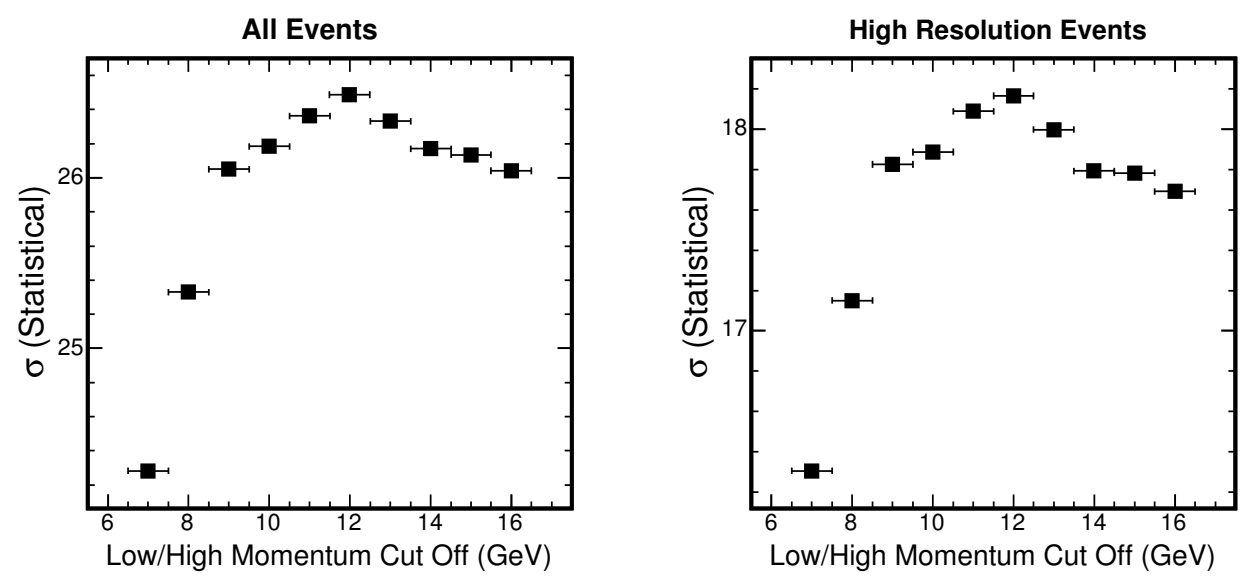

Figure 7.3: Statistical significance of $R_{\text {low } / \text { high }}^{M C}$ from unity as a function of low/high cut off momentum. The highest statistical significance occurs at $12 \mathrm{GeV}$ for both event samples.

likely to bend in the magnetic field of the detector and give a good momentum measurement. On the other hand, higher energy muon tracks appear as straight lines with poor resolution on the momentum measurement. This is reflected by the fact that in the neutrino induced MC, $93 \%$ of the low momentum events in the "all events" sample are also included in the "high resolution" sample. While only $38 \%$ of the high momentum events in the "all events" sample are included in the "high resolution" sample. Thus the "all events" sample has a good separation of low to high momentum muons. Most of the events with a poor momentum measurement from curvature are in the high momentum bin.

Table 7.1 lists the distribution of the data events, as well as the oscillated and unoscillated MC expectation in the low and the high momentum samples. The MC numbers are 
Table 7.1: Comparison between data and neutrino induced Monte Carlo for all events, with best fit Super Kamiokande oscillation parameters $\Delta m^{2}=2.4 \times 10^{-3} \mathrm{eV}^{2}$ and $\sin ^{2}(2 \theta)=$ 0.92 [18. The total background quoted is calculated in Section 6.4

\begin{tabular}{|c|c|c|c|c|}
\hline & Data & Oscillation Expectation & No Oscillation & Background \\
\hline \hline Low Momentum & 45 & 51.0 & 75.1 & 3.6 \\
\hline High Momentum & 103 & 97.9 & 107.5 & 0.7 \\
\hline \hline
\end{tabular}

scaled down by the ratio of data to MC live time. In the data, the ratio of the low to high momentum events is given by

$$
R_{\text {low } / \text { high }}^{\text {data }}=\sum_{\text {low }}\left(N_{\mu}\right) / \sum_{\text {high }}\left(N_{\mu}\right)=0.44_{-0.07}^{+0.08}(\text { stat })
$$

where the sum over low includes events in the low momentum sample, and the sum over high includes events in the high momentum sample. The upper and lower statistical errors account for the Poisson fluctuations in the data [79]. The method used to calculate the upper and lower error was used in the Soudan 2 experiment [80. The limit is determined by observing the random fractional distribution of the ratio of the low to high momentum events in a MC in which Poisson fluctuations are generated around the measured values of low and high momentum events (45 and 103). The lower and the upper limits are set where the combined interval bounds $68 \%$ of the total distribution. This is illustrated in Figure 17.4. The plot on the left shows the random MC distributions with Poisson probability functions for the numerator and the denominator of Equation [7.2. The plot on the right shows the fractional distribution of low to high momentum events with the 

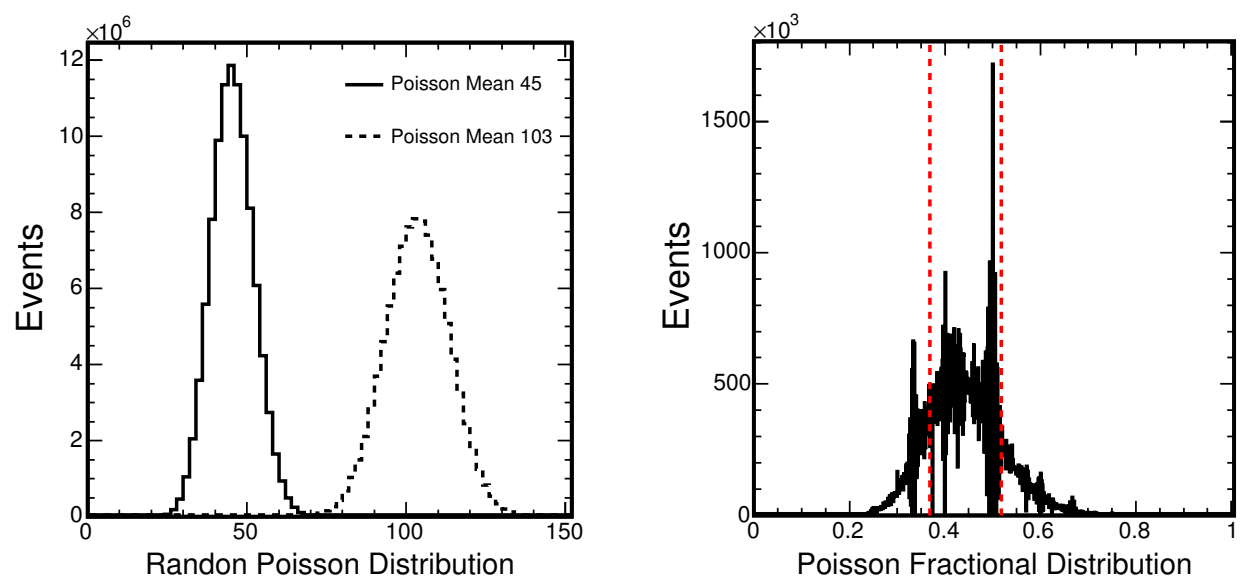

Figure 7.4: Calculation of Poisson confidence limits. The plot on the left shows the random Poisson distribution using a Monte Carlo sample of size $10^{8}$ each. The means of the distributions are the numerator and denominator of Equation 7.2 which are 45 and 103 respectively. The plot on the right shows the fractional distribution of the random Poisson numbers generated by the Monte Carlo sample. The dashed lines indicate the $68 \%$ confidence limit.

same MC sample. The spikes of the figure on the right are the result of the discrete integer values of the Poisson numbers. For example, the spike at 0.5 manifests due to the fact that the distribution of the high momentum events is approximately twice that of the low momentum events. The vertical dashed lines bound the interval that contains $68 \%$ of the fractional distribution.

The ratio for the Monte Carlo sample is similarly defined with the addition of background,

$$
R_{\text {low } / \text { high }}^{M C}=\sum_{\text {low }}\left(N_{\mu}+N_{B G}\right) / \sum_{\text {high }}\left(N_{\mu}+N_{B G}\right)=0.73
$$

where the subscript $B G$ stands for number of muon background events. The statistical 
error in the MC ratio is assumed to be negligible. The error in the background is small and is ignored here since the error will be insignificant compared to the statistical error on the data ratio. In the presence of oscillation, the data to Monte Carlo double ratio is expected to be significantly less than unity. From Table 7.1 the ratio is

$$
R_{A}=\frac{R_{\text {low } / \text { high }}^{\text {data }}}{R_{\text {low } / \text { high }}^{M C}}=0.60_{-0.10}^{+0.11}(\text { stat })
$$

where the subscript A stands for "all events". The statistical error for $R_{A}$ is determined by scaling the error for the data ratio to the $\mathrm{MC}$ ratio, assuming the error for the $\mathrm{MC}$ ratio is negligible compared to the data ratio. The ratio in Equation 7.4 deviates from the unoscillated expectation of unity by $3.6 \sigma$ (statistical).

\subsubsection{Low to High Momentum Ratio - High Resolution Events}

The high resolution events are selected based on their measured momentum from the curvature of the track and the measured error associated with it. From Section 6.3.3, events with $|p / \delta p| \geq 2.5$ have well resolved momenta and identified charge . Table 7.2

Table 7.2: Comparison between data and neutrino induced Monte Carlo for high resolution events, with best fit Super Kamiokande oscillation parameters $\Delta m^{2}=2.4 \times 10^{-3}$ and $\sin ^{2}(2 \theta)=0.92[18$. The total background quoted is calculated in Section 6.4.

\begin{tabular}{|c|c|c|c|c|}
\hline & Data & Oscillation Expectation & No Oscillation & Background \\
\hline \hline Low Momentum & 42 & 48.5 & 70.0 & 3.0 \\
\hline High Momentum & 40 & 38.4 & 40.3 & 0.2 \\
\hline \hline
\end{tabular}


shows the totals for the high resolution event sample. As mentioned earlier, the majority of the low momentum events fall in the high resolution events sample. Comparing the data and the "No Oscillation" expectation from Table 7.2 and 7.1 , there is a 7\% reduction for both the data and unoscillated Monte Carlo in the low momentum sample.

The ratio of low to high momentum muons for the high resolution sample is calculated similarly to the previous section. For the data the ratio is given by

$$
R_{\text {low } / \text { high }}^{\text {data }}=\sum_{\text {low }}\left(N_{\mu}\right) / \sum_{\text {high }}\left(N_{\mu}\right)=1.05_{-0.21}^{+0.26}(\text { stat })
$$

where the sum includes both $\mu^{+}$and $\mu^{-}$. The asymmetry of the statistical errors is due to the Poisson fluctuations of the discrete data values [79]. The $1 \sigma$ statistical errors are computed as described in Section 7.2.2. The ratio for the Monte Carlo is calculated in a similar fashion with added background,

$$
R_{(\text {low } / \text { high })}^{M C}=\sum_{\text {low }}\left(N_{\mu}+N_{B G}\right) / \sum_{\text {high }}\left(N_{\mu}+N_{B G}\right)=1.80
$$

where the subscript $B G$ refers to the estimated background. The error in the background is small, and will be ignored for the ratio calculation. The resulting ratio of ratios is given by

$$
R_{H R}=\frac{R_{\text {low } / \text { high }}^{\text {data }}}{R_{\text {low } / \text { high }}^{M C}}=0.58_{-0.11}^{+0.14}(\text { stat }),
$$

where the subscript $H R$ stands for the high resolution events. The errors are larger for 
the high resolution sample since the number of data events is smaller in this sample than in the sample with all events. The ratio in Equation 7.7 deviates from the unoscillated expectation of unity by $3.1 \sigma$ (statistical). The inclusion of systematic uncertainty will decrease this significance and is discussed in the following section.

\subsection{Systematic Uncertainty}

The systematic uncertainties due to reconstruction have been summarized in Section 6.5. Table 6.4 showed the fractional uncertainties of the individual sources. The calculation for the systematic uncertainty accounts for the difference in the data and MC distributions. An additional source is added for the data sample containing all muon events due to the low to high momentum boundary at $12 \mathrm{GeV}$. The effect of this cut is much smaller for the high resolution sample since the events' momenta are well measured. For the "all events" sample, however, a small fraction of the events' momenta are not well measured around the low to high momentum boundary.

A fractional uncertainty added for the low to high momentum boundary cut would allow us to observe the change in the $\mathrm{MC}$ ratio and subsequently observe the change in the data to $\mathrm{MC}$ ratio. To estimate the fractional uncertainty for the $12 \mathrm{GeV}$ low to high momentum boundary cut, stopping muons from the data and the MC are compared. The momentum for the stopping muons is measured by its range. The track momentum from its range is determined by taking the average energy loss per plane in order to accurately measure the momentum. The momentum from range measurement for the 
data is compared to that of the MC sample. A sample of 11,000 stopping muon events from data and approximately 4,500 stopping neutrino induced MC events are selected for the comparison. In the momentum distribution calculated from range, $95 \%$ of data stopping muons have measured momenta below $12 \mathrm{GeV}$, while $93 \%$ of the MC stopping muons are below $12 \mathrm{GeV}$. This is a difference of $2 \%$ from data to MC. However, not all the muons are stopping events in the low energy range. To account for the events with the momentum measurement from the magnetic field, a conservative $3 \%$ fractional uncertainty is assigned to the low to high momentum boundary cut for the "all events" sample.

There are several sources of systematic uncertainty in the physics simulation. The overall normalization of the neutrino flux is the largest contributor to the systematic uncertainty. This will be taken into account in the fit analysis. The normalization uncertainty does not effect the ratio calculation since the flux normalization scales the low and the high momentum events by a constant and gets canceled out for the ratio calculation. As described in Section 5.3.2 the total estimated uncertainty arising from the primary cosmic ray flux is $\pm 25 \%[52]$.

The primary spectrum of the atmospheric neutrinos has an energy dependence that is proportional to $E^{-(\gamma+1)}$, where $\gamma$ is the "spectral index" and has a mean value of $1.7 \pm 0.05$ [76], a 3\% deviation. There are additional uncertainties due to neutrino and anti-neutrino cross-sections. For the rate of muons with neutrino energies below $30 \mathrm{GeV}$ there is a $\pm 7 \%$ [77. uncertainty due to the neutrino and anti-neutrino cross sections. Similarly, a $\pm 2 \%$ uncertainty is assigned to the rate of muons coming from neutrinos with energies above 
$30 \mathrm{GeV}$ [8].

Table 7.3: Systematic uncertainty for the low to high momentum double ratio

\begin{tabular}{|l|c|c|c|}
\hline & & All Events & High Resolution Events \\
\hline Source & Fractional $\sigma$ & $\Delta R_{i}$ & $\Delta R_{i}$ \\
\hline Reconstruction systematics: & & & \\
\hline \hline$\chi_{\text {fit }}^{2} / n d f$ & 0.04 & 0.0 & 0.0 \\
\hline$\chi_{\text {new-fit }}^{2} / n d f$ & 0.03 & 0.0 & 0.0 \\
\hline Time vs. Y Correlation & 0.03 & 0.0 & 0.0 \\
\hline Track Time Digits & 0.04 & 0.01 & 0.01 \\
\hline$|p / \delta p|$ & 0.05 & 0.0 & 0.02 \\
\hline Low to High Momentum Cut Off & 0.03 & 0.02 & 0.0 \\
\hline \hline Physics Model systematics: & & & 0.0 \\
\hline Normalization & 0.25 & 0.0 & 0.04 \\
\hline Spectral Index & 0.03 & 0.07 & 0.02 \\
\hline Cross Section $(\mathrm{E} \leq 30 \mathrm{GeV})$ & 0.07 & 0.02 & 0.01 \\
\hline Cross Section $(\mathrm{E}>30 \mathrm{GeV})$ & 0.02 & 0.01 & 0.05 \\
\hline Total $\Delta R=\sqrt{\sum\left(\Delta R_{i}\right)^{2}}$ & & 0.08 & \\
\hline \hline
\end{tabular}

The fractional uncertainties due to reconstruction and physics modeling are used to calculate the absolute uncertainty in the final ratio. The parameters are individually changed in the MC by the amount listed as "fractional $\sigma$ " in Table $\mathbf{7 . 3}$ to calculate the change in the $\mathrm{MC}$ ratios and in the final ratios, $R_{A}$ and $R_{H R}$. The MC ratios are altered by changing the "plus" systematics of the parameters individually. The data ratio is then divided by the altered $\mathrm{MC}$ ratio to calculate $+\Delta R$ for both event samples. Similarly, $-\Delta R$ is calculated by changing the MC ratio with the "minus" systematics individually. Finally the $\Delta R$ are calculated by taking the average of the $+\Delta R$ and the $-\Delta R$. The results are summarized in Table 7.3 
The majority of the selection cuts do not have any significant effect on the final ratio. Varying the number of timing digits for the muon tracks from 31-33 digits (32 digits nominal) yields $\Delta R=0.01$. Varying charge/momentum selection cut from Section 6.3.3 from 1.4 to 1.6 (1.5 nominal) yields $\Delta R=0.01$. This cut is not imposed on the sample containing all muon events and hence does not contribute to the systematic uncertainty on the final ratio. Varying the cut off momentum by $\pm 3 \%$ for the "all events" sample gives $\Delta R=0.02$.

For the physics modeling, the largest fractional uncertainty comes from the flux normalization $( \pm 25 \%)$. However, as described earlier, the uncertainty on the final ratio cancels out since the normalization scales the number of low and high muon momentum by the same constant. Varying the spectral index from 1.65 to $1.75(\gamma=1.7$ nominal $)$ yields $\Delta R=0.07$ for the sample with all events, and $\Delta R=0.04$ for high resolution events. The systematic uncertainty is greater for the "all events" sample since the number of higher momentum muons, and hence higher energy neutrinos, is higher than that in the sample of high resolution events. Spectral index variation is greater at high energies than at low energies. Varying the cross-section for $\mathrm{E}<30 \mathrm{GeV}$ gives $\Delta R=0.02$ and varying the cross-section for $\mathrm{E}>30 \mathrm{GeV}$ gives $\Delta R=0.01$ for the final ratio. The total systematic uncertainty is calculated by adding the individual contribution in quadrature. This yields $\Delta R_{A}($ syst $)=0.08$, where A stands for the "all events" sample, and $\Delta R_{H R}($ syst $)=0.05$, where $H R$ stands for the high resolution sample.

From Equations 7.4 and 7.7 and Table[7.3 the ratio of the low to high muon momentum 
ratio for the data to the low to high muon momentum ratio for the Monte Carlo is

$$
R_{A}=\frac{R_{\text {low } / \text { high }}^{\text {data }}}{R_{\text {low } / \text { high }}^{M C}}=0.60_{-0.10}^{+0.11}(\text { stat }) \pm 0.08(\text { syst }),
$$

and

$$
R_{H R}=\frac{R_{\text {low } / \text { high }}^{\text {data }}}{R_{\text {low } / \text { high }}^{M C}}=0.58_{-0.12}^{+0.15}(\text { stat }) \pm 0.05(\text { syst }),
$$

where $R_{A}$ and $R_{H R}$ are the ratios for the "all events" muon sample and the high resolution muon sample, respectively. The first sample has higher statistics and hence lower statistical error, while the high resolution sample has a lower systematic error. Adding the systematic error with the upper statistical confidence limit in quadrature gives a total error of $\Delta R_{A}=0.14$ for the sample with all events. Similarly, the total error for the ratio with high resolution muons is $\Delta R_{H R}=0.16$. For the sample with all events, the ratio differs from the unoscillated expectation of unity by $2.9 \sigma$. For the sample containing only high resolution muons, the ratio differs from unoscillated expectation of unity by $2.6 \sigma$. Both results are consistent with the neutrino oscillation hypothesis.

So far, the double ratios calculated give a strong indication for the neutrino oscillations. In the following section, the data, binned in zenith angle and momentum, is compared to the MC expectation in order to improve the measurement of the oscillation parameters. 


\subsection{Neutrino Oscillation Fit}

For the oscillations suggested by the ratios in Sections 7.2.2 and 7.2.3, the data and Monte Carlo samples are compared in the presence of oscillation. To perform the fit, the low and high momentum samples are divided into 4 equal bins in the vertex $y$ direction cosine, $d \cos y$, where $d \cos y$ is the negative cosine of the zenith angle, ranging from -0.06 to 1.0 . A grid search is performed over the parameter space $\Delta m^{2}$ and $\sin ^{2} 2 \theta$. At every grid point the observed data is compared to the Monte Carlo prediction with the parameters values, and a log-likelihood is calculated as follows:

$$
\ln \mathcal{L}=\sum_{i=1}^{n}\left(N_{i}^{D} \ln N_{i}^{M C}-N_{i}^{M C}\right)-\frac{\alpha^{2}}{2 \sigma^{2}}
$$

where, $i$ iterates over 8 bins; 2 bins in momentum: low momentum $\left(1 \mathrm{GeV}<p_{\mu} \leq 12\right.$ $\mathrm{GeV})$ and high momentum $\left(p_{\mu}>12 \mathrm{GeV}\right)$, and 4 equally spaced bins in $d \cos y$ for each momentum bin. $N_{i}^{M C}$ are the predicted number of events in the $i^{t h}$ bin, $N_{i}^{D}$ are the observed number of events in the $i^{\text {th }}$ bin, $\alpha$ is the Gaussian variation of the neutrino flux normalization around zero, and $\sigma$ is the systematic error in the neutrino flux normalization. The predicted number, $N_{i}^{M C}$, at each point in the parameter space is given by,

$$
N_{i}^{M C}=(1+\alpha) P_{i}+B G_{i}
$$


where, $B G_{i}$ are the estimated background in the $i^{t h}$ bin and $P_{i}$ are the predicted number of neutrino induced muon events in the $i^{\text {th }}$ bin calculated using the survival probability function for the neutrinos. As derived in Chapter 2] the survival probability $P_{s}$ for the dominant two flavor oscillation $\nu_{\mu} \rightarrow \nu_{\tau}$ is given by

$$
P_{s}=1-P_{o}\left(\nu_{\mu} \rightarrow \nu_{\tau}\right)=1-\sin ^{2}\left(2 \theta_{23}\right) \sin ^{2}\left(\frac{1.27 \Delta m_{23}^{2} L}{E}\right)
$$

where $P_{o}$ is the probability of oscillation, $L$ is the distance traveled by the neutrinos from their production in the atmosphere $(\mathrm{km})$, and $E$ is their energy in $\mathrm{GeV} . L$ is calculated from the reconstructed muon direction, projecting it back towards its production height (presumed constant), and is given by,

$$
L=\sqrt{(R-y)^{2}+(y+h)(2 R-y+h)}-(R-y) \cos \theta
$$

where, $R$, taken as $6370 \mathrm{~km}$, is the radius of the Earth, $\theta$ is the zenith angle of the reconstructed muon, $y$, assigned $0.8 \mathrm{~km}$, is the depth of the MINOS Far Detector, and $h$, assumed $20 \mathrm{~km}$, is the neutrino production height in the atmosphere from the surface of the Earth.

For the fit analysis, only the dominant systematic uncertainty due to normalization $( \pm 25 \%)$ is included in the likelihood calculation. The rest of the systematic uncertainties are smaller and are ignored here. The normalization variable $\alpha$ in Equation 7.11 is allowed 
to vary freely around zero at every grid point of the parameter space. The likelihood, however, is constrained by adding a Gaussian penalty term, $\alpha^{2} / 2 \sigma^{2}$. The likelihood function is maximized with respect to $\alpha$ at each grid point of $\Delta m^{2}$ and $\sin ^{2} 2 \theta$. The maximum likelihood is found at $\Delta m^{2}=1.3 \times 10^{-3} \mathrm{eV}^{2}$ and $\sin ^{2} 2 \theta=1.0$. The best fit normalization for these parameter values is $(1+\alpha)=0.94$.
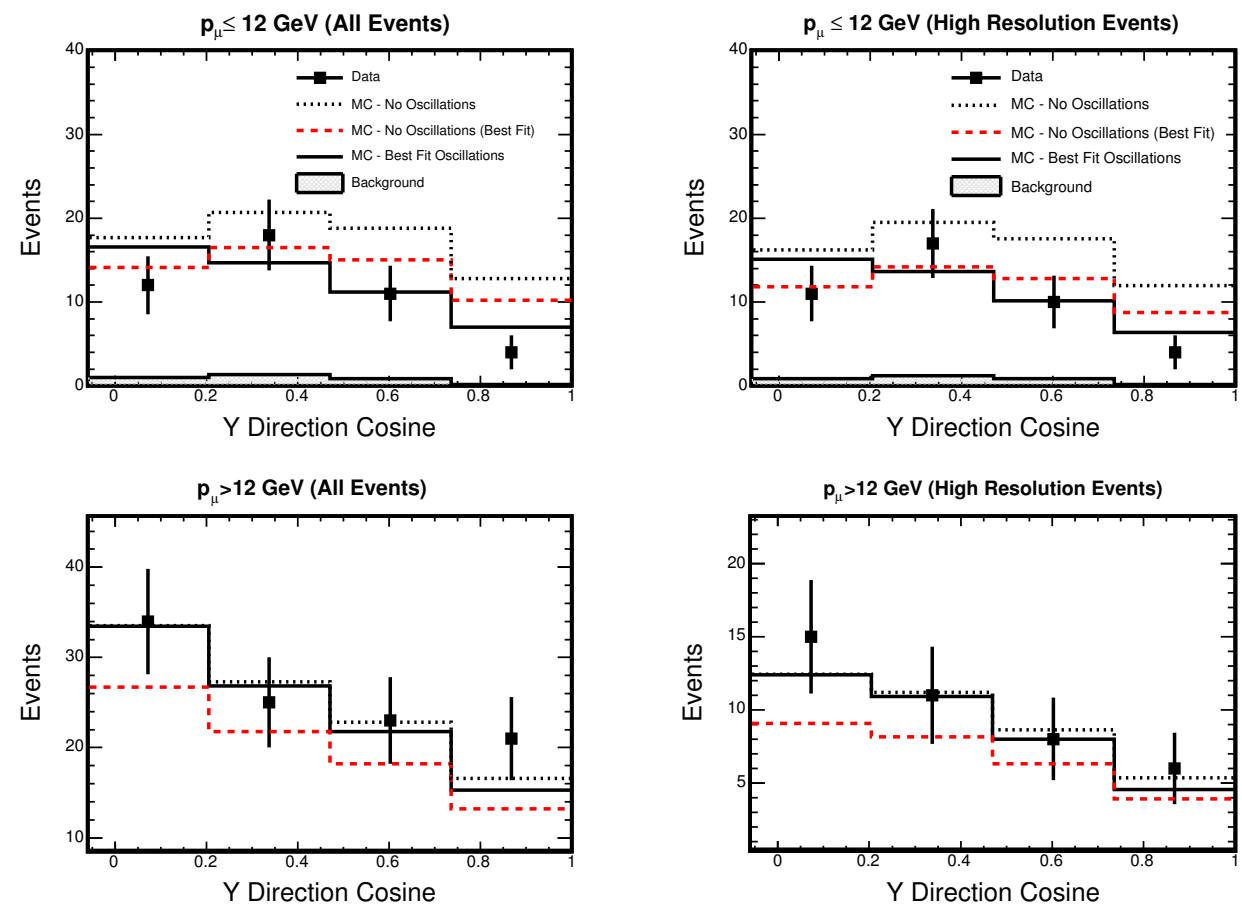

Figure 7.5: The distribution of neutrino induced muons as a function of the $y$-direction cosine (negative cosine of the zenith angle). The top two plots contain the distributions of muon with momenta $12 \mathrm{GeV}$ or less. The oscillation effect is the greatest for these muons as suggested by the low to high momentum ratios calculated in Sections 7.2.2 and 7.2.3

Figure [7.5 shows the momentum separated event distributions for both event samples as a function of $y$ direction cosine (negative cosine of zenith angle). The best oscillation fit 
to the data is shown as the solid line. The unoscillated expectation (dotted line) shows the unoscillated MC prediction scaled by the normalization of the oscillated best fit (94\%). A separate null oscillation fit to the data is performed and is discussed shortly. The oscillation effects are more pronounced for muons with lower energy as suggested by the low to high momentum ratio calculations in Sections 7.2 .2 and 7.2.3. The null oscillations hypothesis prediction fits data for high momentum muons much better than the low momentum muons for both the event samples. Figure [7.6 shows the the best fit contour values for
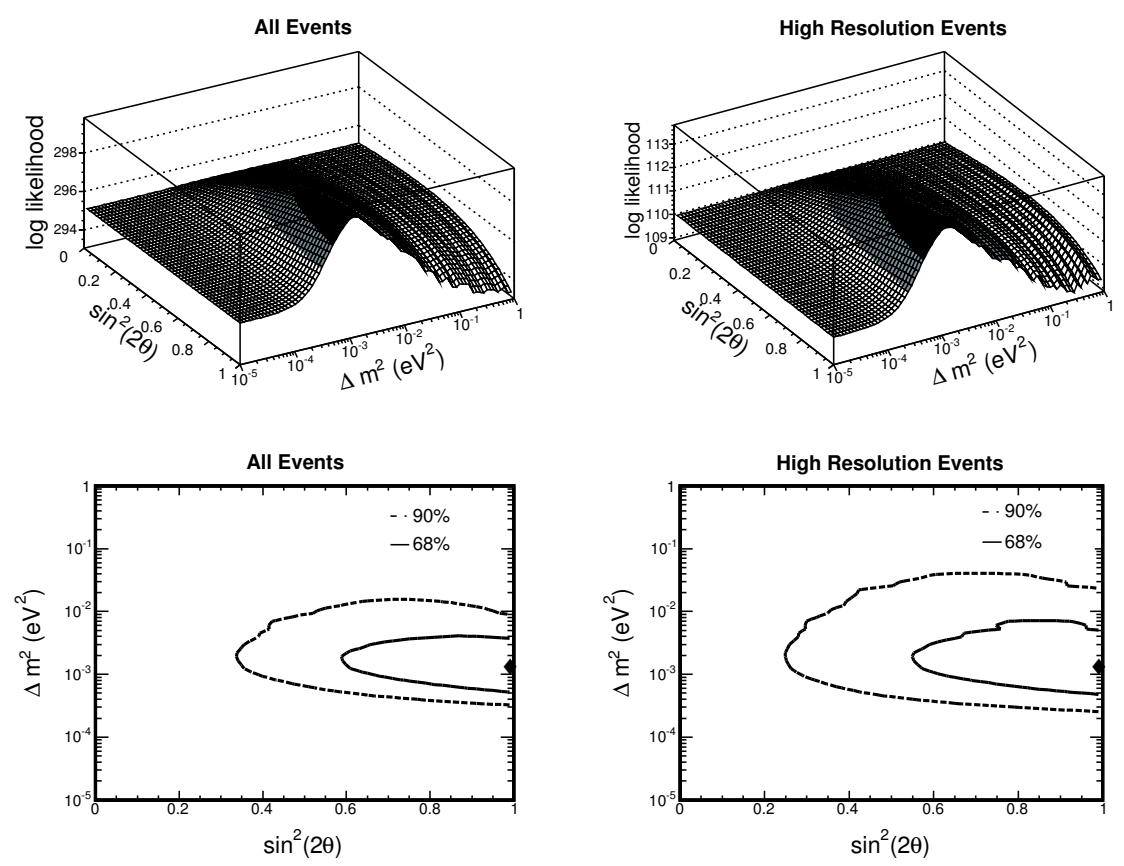

Figure 7.6: Log-likelihood contours. The top plots show the maximum log-likelihood values calculated from Equation 7.10, The $68 \%$ and the $90 \%$ contours are shown in the bottom two plots.

the sample with all events and the sample with high resolution events. The solid and the 
dashed contour lines indicate the $68 \%$ and $90 \%$ confidence limits respectively. The limits were calculated by taking the difference between the log-likelihood for the best fit value and the log-likelihood of the individual grid points. The $68 \%$ and $90 \%$ contour levels were set at $\Delta \ln \mathcal{L}$ values of 1.2 and 2.3 respectively.

To observe how the unoscillated MC fits to the data, a separate fit for the null oscillation hypothesis to the data is performed. The procedure for the fit is the same as the oscillated MC to data fit with the exception that $P_{o}\left(\nu_{\mu} \rightarrow \nu_{\tau}\right)$ from the Equation [7.12] is set to zero for all the predicted values. The unoscillated MC normalization is allowed to vary freely and the likelihood is constrained with a penalty term as before. The solid line in Figure [7.5 shows the unoscillated best fit for both the event samples. The differences between the best fit log-likelihood for the oscillated MC and the best fit log-likelihood for the unoscillated MC, $\Delta \ln \mathcal{L}$, are 4 and 3.5 for the sample with all events and the sample with high resolution events, respectively. Thus the null oscillation hypothesis prediction is excluded at $>95 \%$ confidence level for both the event samples. The best fit values and the allowed regions obtained are compatible to the Super Kamiokande result [18], and the best fit results from the MINOS contained analysis [74].

The contour levels determined with the likelihood analysis appear small compared to the MINOS contained atmospheric neutrino analysis [74]. The contained analysis had a number of events comparable to this analysis. It used the measured $L / E$ values to generate the contours. The $L / E$ analysis contains more measured informations and can, in principle, constrain the allowed region better than the zenith angle analysis. Figure 


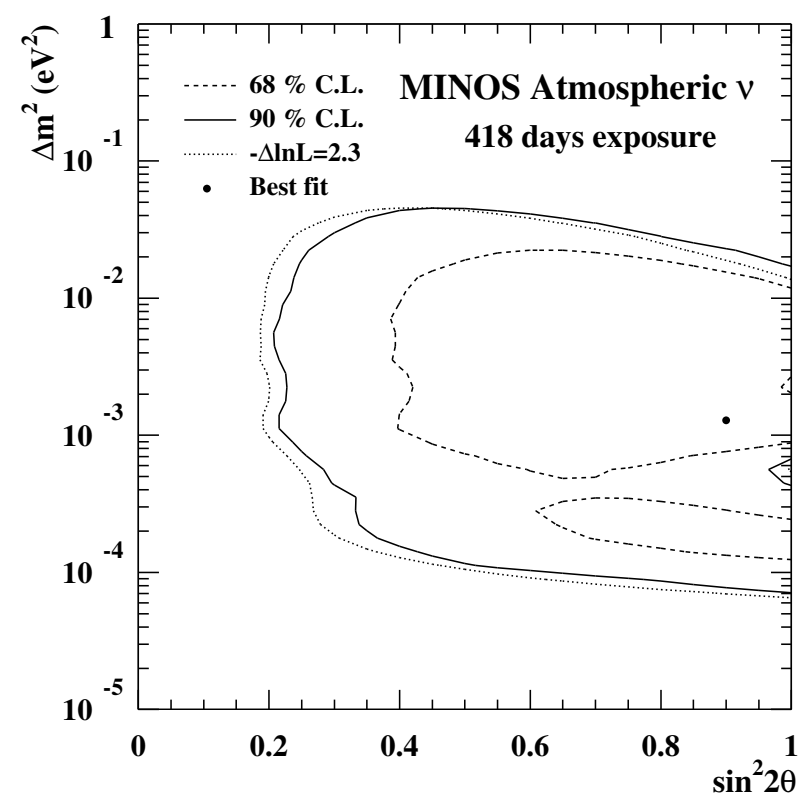

Figure 7.7: Log likelihood contours for the MINOS contained atmospheric analysis [74].

7.7 shows the $68 \%$ and the $90 \%$ log likelihood contours for that analysis. In order to understand the smallness of the $90 \%$ contour levels, the MC sample is used to determine the parametric sensitivity of the double ratios.

In order to test the sensitivity of the ratios from Sections 7.2.2 and 7.2.3 $R_{A}$ and $R_{H R}$ are calculated using the neutrino induced MC for the "all events" sample and the high resolution sample in the parameter space of $\Delta m^{2}$ and $\sin ^{2} 2 \theta$. For both the event samples, the ratio of low to high muon momentum (scaled to data) is calculated for oscillated Monte Carlo for each value of $\Delta m^{2}$ and $\sin ^{2} 2 \theta$. The ratio of ratios, $R_{A}^{M C}$ and $R_{H R}^{M C}$, of the "all events" sample and the high resolution sample respectively are calculated by dividing 

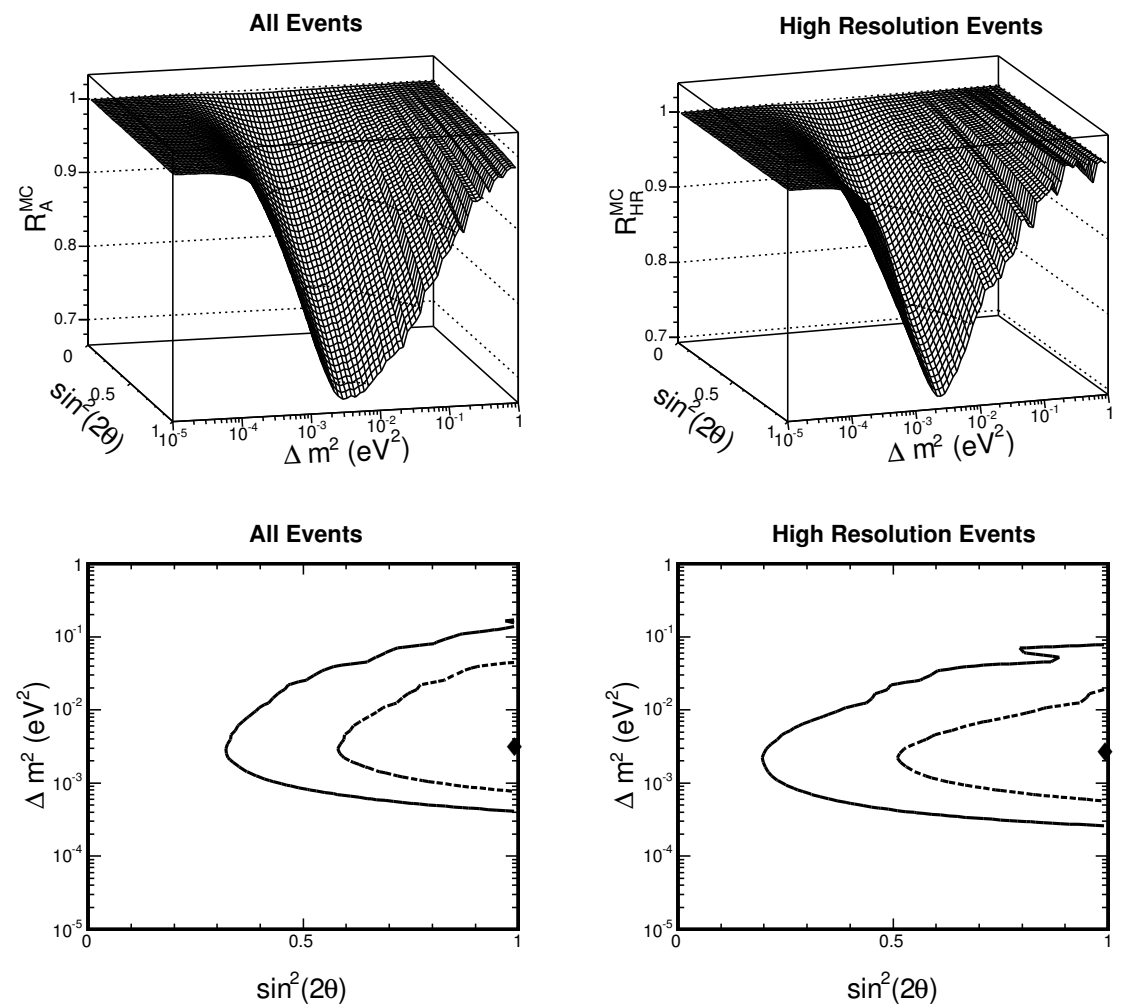

Figure 7.8: The low to high momentum ratio sensitivity. The top two plots show the behavior of the calculated ratio in the parameter space for the two event samples. The bottom plots show the contours for the $68 \%$ (dashed) and the $90 \%$ (solid) statistical errors on the ratios.

the oscillated ratio by the unoscillated ratio. A total of eighty values of $\Delta m^{2}$ ranging from $10^{-5} \mathrm{eV}^{2}$ to $1.0 \mathrm{eV}^{2}$ and fifty values of $\sin ^{2} 2 \theta$ ranging from 0 to 1 are selected for the ratio sensitivity calculation. Figure 7.8 shows the ratio of the oscillated ratio to the unoscillated ratio as a function of $\Delta m^{2}$ and $\sin ^{2} 2 \theta$. For the low values of $\Delta m^{2}$ and $\sin ^{2} 2 \theta$, which correspond to low values of oscillation probability, the $\mathrm{MC}$ double ratios are close to unity since neither the low momentum muons nor the high momentum muons are oscillated 
away. On the other hand, for the high values of $\Delta m^{2}$ and $\sin ^{2} 2 \theta$, which correspond to high values of oscillation probability for both the low and the high momentum muons, both the low and the high momentum muons are oscillated away and the double ratios converge to unity. The smallest values of the ratios are marked with diamonds and the contours for the $68 \%$ and the $90 \%$ statistical error on the ratio are indicated by the dashed and solid lines, respectively. The errors come from the oscillated low to high momentum values in the $\mathrm{MC}$ that have been scaled to the data exposure. The smallest values of the ratios are $R_{A}^{M C}=0.68 \pm 0.12($ stat $)$ and $R_{H R}^{M C}=0.71 \pm 0.16($ stat $)$, and occur at $\sin ^{2} \theta$ at 1 , and $\Delta m^{2}=3.0 \times 10^{-3} \mathrm{eV}^{2}$ for $R_{A}$ and $\Delta m^{2}=2.6 \times 10^{-3} \mathrm{eV}^{2}$ for $R_{H R}$, respectively.

The lowest possible values of the MC oscillated to MC unoscillated ratios are somewhat higher than the data to MC ratios calculated in Sections 7.2 .2 and 7.2.2 where $R_{A}$ and $R_{H R}$ are $0.60_{-0.10}^{+0.11}$ (stat) and $0.58_{-0.12}^{+0.15}$ (stat) respectively. Although the data to MC ratios are within the errors of the $\mathrm{MC}$ ratio of ratios, it seems that a fluctuation in the data is pulling the data to MC ratios below the physically allowed values for the both events samples. To investigate further, a log likelihood analysis is performed comparing data to MC as described before. For this log-likelihood analysis, the values of $\sin ^{2} 2 \theta$ are set to vary from 0.0 to 2.0. That is, the parameter values are stretched beyond the allowed and into the non-physical region. Figure 7.9 shows the results of the log-likelihood calculations for both event samples. The best fit $\sin ^{2} 2 \theta$ value is, in fact, in the unphysical region for both event samples. This is interpreted in the following way. The data to MC low to high momentum ratio is smaller than that of the pure $\mathrm{MC}$ ratios. In order for the oscillated 

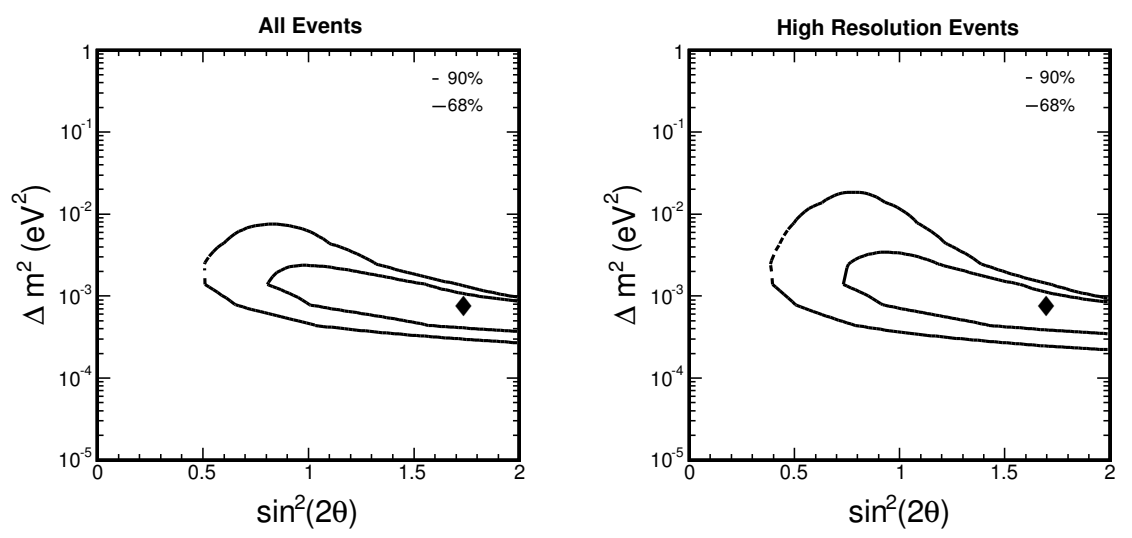

Figure 7.9: The log-likelihood contour plots extended into the unphysical region.

MC predictions in the likelihood analysis to fit to the data, the MC ratios are suppressed further. This can only be done by higher values of $\sin ^{2} 2 \theta$ than in the allowed region. The area bounded by the $90 \%$ contours is bigger relative to the best fit points, and is seen to be more comparable to the contained analysis.

\section{Summary of the Oscillation Results}

The oscillations suggested by the double ratios calculated in Sections [7.2.2 and [7.2.3 have been fit in the oscillation parameter space. The best fit results obtained agree with the MINOS contained analysis and the Super Kamiokande oscillation analysis. The combined energy and the zenith angle oscillation fit shows more suppression for the low momentum events than for the high momentum events, confirming the oscillations suggested by the data to MC double ratios. The oscillation effects are greater for higher values of zenith 
angle (greater neutrino baseline), than lower ones. This shows evidence of neutrino disappearance for higher values of neutrino baseline as suggested by the survival probability in Equation 7.12 The null oscillation hypothesis is rejected at $>95 \%$ confidence level by allowing the unoscillated MC to be fit to the data. The $90 \%$ contour plots generated by the maximum log-likelihood are smaller than the MINOS contained analysis contours. The pure MC low to high momentum (oscillated) ratio to MC low to high momentum (unoscillated) ratio is higher than the data to $\mathrm{MC}$ double ratios suggesting a fluctuation in data that is pulling the ratio below the allowed values. An extended likelihood done for the unphysical region reveals that the best $\sin ^{2} 2 \theta$ is higher than one. This demonstrates that the MC predictions are better matched to data with a higher value of $\sin ^{2} 2 \theta$. This also indicates why the $90 \%$ contour levels are smaller than the MINOS contained results.

\subsection{Test of CPT Conservation}

MINOS is the first underground detector to be able to distinguish $\mu^{+}$from $\mu^{-}$from the curvature of the track due to the presence of an average magnetic field of $1.3 \mathrm{~T}$. In this section the CPT conservation is tested, which requires that the probability of an atmospheric $\nu_{\mu}$ oscillation is the same rate as that of an $\bar{\nu}_{\mu}$ oscillation. The ratio of $\mu^{-}$

to $\mu^{+}$of data compared to the same ratio of Monte Carlo, combined with the associated errors should provide a test for the CPT conservation. 


\subsubsection{Charge Ratio Determination}

In order to determine the $\mu^{-}$to $\mu^{+}$ratio for the data and the Monte Carlo, the high resolution sample is selected based on the selection cut in Section 6.3.3. Events that have measured momenta of two and a half times the measured error are kept as high resolution events $(|p / \delta p| \geq 2.5)$. The events in this sample have well measured momenta and identified charge. Table 7.4 shows the charge separated event distribution. In the

Table 7.4: Charge separated event distribution

\begin{tabular}{|l|c|c|c|}
\hline & Data & MC (No Oscillation) & Background \\
\hline \hline$\mu^{-}$ & 52 & 73.0 & 1.8 \\
\hline$\mu^{+}$ & 30 & 37.3 & 1.4 \\
\hline \hline
\end{tabular}

data the charge ratio is calculated as follows:

$$
R_{\mu^{-} / \mu^{+}}^{\text {data }}=\sum\left(N_{\mu-}\right) / \sum\left(N_{\mu+}\right)=1.73_{-0.35}^{+0.47}(\text { stat })
$$

The asymmetry of the statistical error is due to the discreteness of the data values [79]. The technique for the calculation has been described in Section 17.2.2. The Monte Carlo ratio is calculated similarly with the addition of the estimated background,

$$
R_{\mu^{-} / \mu^{+}}^{M C}=\sum\left(N_{\mu-}+N_{\mu-}^{B G}\right) / \sum\left(N_{\mu+}+N_{\mu-}^{B G}\right)=1.93
$$


where the superscript $B G$ stands for background. The error on the background does not make a significant contribution to the MC error and is ignored for this analysis. The statistical error on the MC ratio is negligible compared to the error on the data ratio. The $\mu^{-}$to $\mu^{+}$ratio for data is compared to same ratio for MC. The following data to MC double ratio is expected to be consistent with unity if CPT is conserved,

$$
R_{C P T}=\frac{R_{\mu^{-} / \mu^{+}}^{\text {data }}}{R_{\mu^{-} / \mu^{+}}^{M C}}=0.90_{-0.18}^{+0.24}(\text { stat }) .
$$

With the calculated statistical error, the ratio is consistent with unity within $1 \sigma$.

\subsubsection{Systematic Uncertainty}

There are several sources of systematic uncertainty for the CPT ratio analysis. The sources due to reconstruction have been identified in Section 6.5] An additional source is added for the charge ratio calculation. The purity of charge selection arises from the charge/momentum cut. The purity of the selected events, from Section 6.3.3. is $97 \%$. Thus a $\pm 3 \%$ fractional uncertainty is assigned for the purity of the high resolution selection cut. The systematic uncertainty in physics modeling has been described in in Section 7.3 . One more source is added to the systematic uncertainty arising from the $\bar{\nu}_{\mu}$ cross section to the $\nu_{\mu}$ cross section ratio. The error in the cross section ratio is estimated to be $\pm 9 \%$ for $\mathrm{E} \nu_{\mu}>$ $30 \mathrm{GeV}$ [77], and $\pm 2 \%$ for $\mathrm{E} \nu_{\mu}<30 \mathrm{GeV}$ [78]. The calculations of systematic uncertainty for the charge ratio follow from the procedure described in Section [6.5. Table 7.5 lists the sources and the systematic errors for $R_{C P T}$. Adding the individual contributions in 
Table 7.5: systematic uncertainty

\begin{tabular}{|l|c|c|}
\hline Source & Fractional $\sigma$ & $\left(\Delta R_{C T P}\right)_{i}$ \\
\hline Reconstruction systematics: & & \\
\hline \hline$\chi_{\text {fit }}^{2} / n d f$ & 0.04 & 0.0 \\
\hline$\chi_{n e w-f i t}^{2} / n d f$ & 0.03 & 0.0 \\
\hline Time vs. Y Correlation & 0.03 & 0.0 \\
\hline Track Time Digits & 0.04 & 0.01 \\
\hline Charge Purity & 0.03 & 0.06 \\
\hline$|p / \delta p|$ & 0.05 & 0.01 \\
\hline \hline Physics Model systematics: & & \\
\hline Normalization & 0.25 & 0.0 \\
\hline Spectral Index & 0.03 & 0.01 \\
\hline Cross Section $(\mathrm{E} \leq 30 \mathrm{GeV})$ & 0.07 & 0.01 \\
\hline Cross Section $(\mathrm{E}>30 \mathrm{GeV})$ & 0.02 & 0.0 \\
\hline $\bar{\nu}_{\mu} / \nu_{\mu}(\mathrm{E}<30 \mathrm{GeV})$ & 0.09 & 0.07 \\
\hline $\bar{\nu}_{\mu} / \nu_{\mu}(\mathrm{E}>30 \mathrm{GeV})$ & 0.02 & 0.01 \\
\hline Total $\Delta R=\sqrt{\sum\left(\Delta R_{i}\right)^{2}}$ & & 0.09 \\
\hline \hline \multicolumn{2}{|l}{}
\end{tabular}

quadrature gives a total systematic error of \pm 0.09 . The upper statistical confidence limit for $R_{C P T}$ is +0.24 . The total error is thus 0.26 . The ratio is thus consistent with CPT conservation at the $1 \sigma$ level. However, the statistical precision of the measurement is limited. More data from the expected 10 year exposure of the experiment may provide evidence of a difference in the rate of neutrino to anti-neutrino oscillations when the number of high resolution events reaches several hundred events.

\subsection{Oscillation Sensitivity and 10 Year Predictions}

The oscillation sensitivity for the data exposure is calculated using the neutrino induced Monte Carlo sample. The Monte Carlo is divided into two samples as before. For the 
two event samples a grid search is performed in the parameter space of $\Delta m^{2}$ and $\sin ^{2} 2 \theta$. Approximately 164 Monte Carlo experiments are performed for each value of the grid. For each value of the parameters, the predicted number of muons using the best fit values of $\Delta m^{2}$ and $\sin ^{2} 2 \theta$ from Section 7.4 is compared to the predicted number of muons using the parameter values of the grid. A log-likelihood is extracted using an equation similar to Equation [7.10]

$$
\ln \mathcal{L}=\sum_{i=1}^{n}\left(N_{i}^{\overline{M C}} \ln N_{i}^{M C}-N_{i}^{M C}\right)-\frac{\alpha^{2}}{2 \sigma^{2}}
$$

where, $N_{i}^{\overline{M C}}$ are the predicted number of muons averaged over 164 Monte Carlo experiments with the best fit values of $\Delta m^{2}$ and $\sin ^{2} 2 \theta$ scaled to the data exposure, $N_{i}^{M C}$ are the predicted number of muons using the values of the grid, $\alpha$ is the normalization variation, and $\sigma= \pm 25 \%$, is the normalization uncertainty at the grid point. Figure 7.10 shows the result of the sensitivity calculation. The best fit point on the grid obviously coincides with the best fit result for the data oscillations analysis since the values of the parameters are exactly the same. The contours were generated by taking the log-likelihood difference of the best fit point from the log-likelihood of the individual grid points. The $68 \%$ (solid line) and the $90 \%$ (dashed line) are set at $\Delta \ln \mathcal{L}$ values of 1.2 and 2.3 , respectively. For the high resolution sample, the $90 \%$ contour is not constrained in the $\Delta m^{2}$ range used. This tendency is also seen in the high resolution contours determined for the $\Delta m^{2}$ window and the extended region for the high resolution sample. The sensitivity log-likelihood in the region where the $90 \%$ contour is not constrained is approximately constant. So 

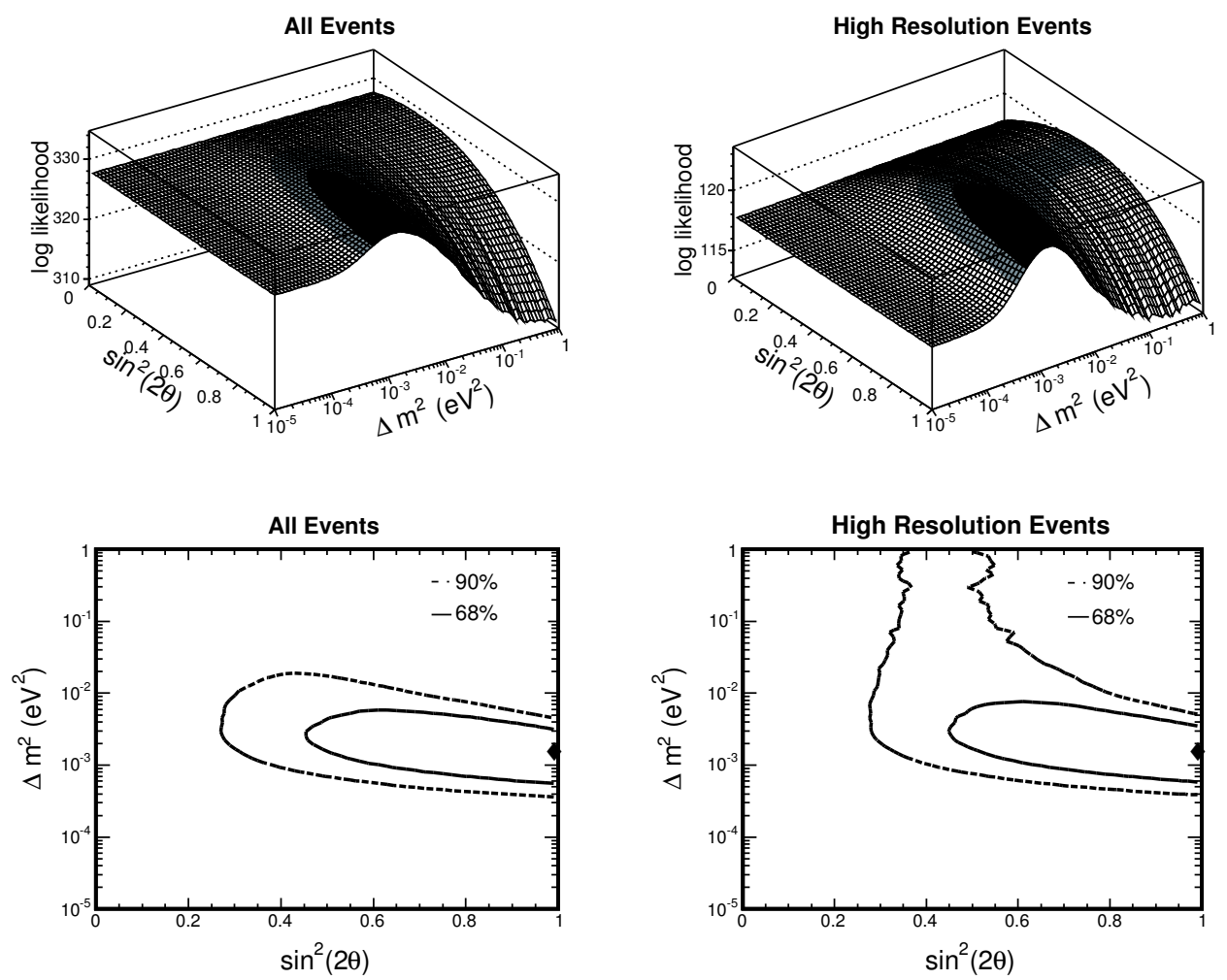

Figure 7.10: Oscillation sensitivity contours determined by using a log-likelihood analysis with the best fit values from data to MC oscillation fit values. The $68 \%$ and the $90 \%$ contour levels are drawn with solid and dashed lines respectively.

the contour itself is sensitive to slight variations of $\Delta \ln \mathcal{L}$. The $90 \%$ contour levels for both event samples are larger compared to the $90 \%$ contours generated with the data to MC oscillation fit. However, when compared to the contours generated by the extended likelihood analysis in the unphysical region, the contours are more compatible. This also confirms that a fluctuation in the data may be pulling the best fit point into regions where $\sin ^{2} 2 \theta$ is greater than one. 

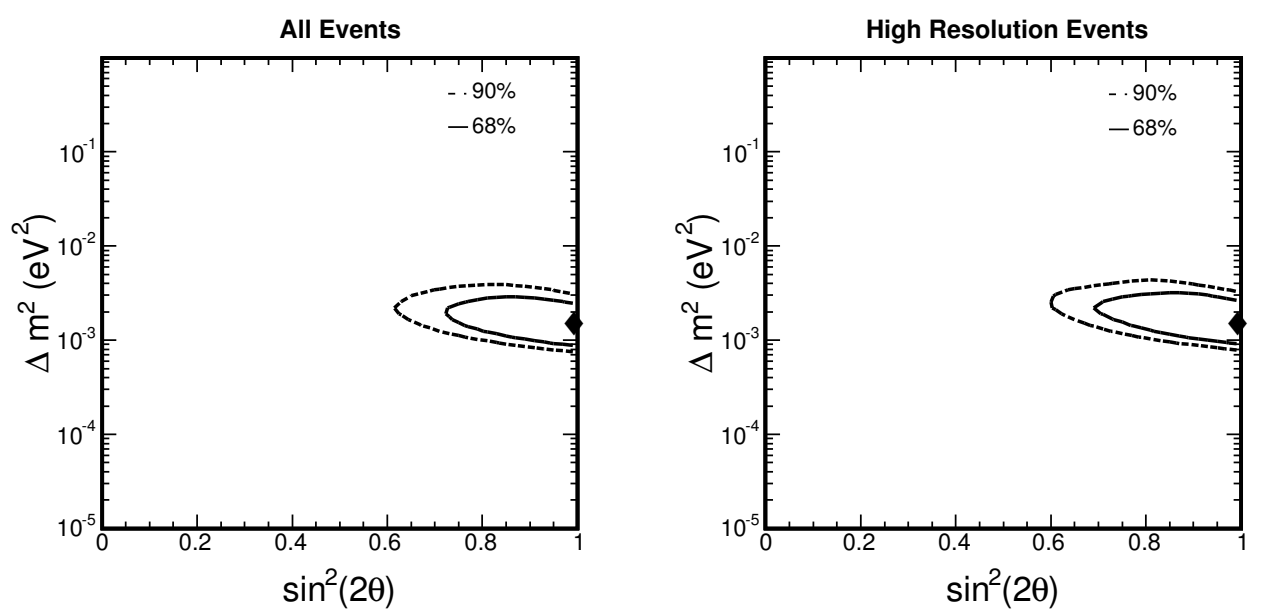

Figure 7.11: Projected 10 year oscillation sensitivity contours determined by using a log likelihood analysis with the best fit values from data to $\mathrm{MC}$ oscillation fit values.

MINOS is expected to collect data until the year 2010 and may be a couple of years beyond. That is approximately 10 years of atmospheric neutrino data. At this rate, the expected total number of neutrino induced muons will be in the order of 600 . This is not competitive with Super Kamiokande Collaboration's current data, however, having a large data sample will constrain the parameter space around the best fit much better. Figure 7.11 shows the MINOS sensitivity for a 10 year data collection. The contours are much smaller as expected. The uncertainties on the best fit point are much smaller at maximal mixing. The parameter values at $90 \%$ C. L. are $\left(8 \times 10^{-4} \mathrm{eV}^{2}<\Delta m^{2}<3 \times 10^{-3} \mathrm{eV}^{2}\right)$, and $\sin ^{2} 2 \theta>0.6$. Thus the expected results will be more precise than the current data.

Similarly, a 10 year prediction for the CPT charge ratio can be made using the high resolution sample. The expected number of neutrino induced $\mu^{-}$and $\mu^{+}$will reach several 
hundred. This will minimize the statistical uncertainty by a factor of approximately 4 . Assuming that the current $\mathrm{CPT}$ data to MC double ratio (from Equation [7.16) remains constant, the expected ratio will be,

$$
R_{C P T}(10 \text { years })=0.90 \pm 0.05 \text { (stat) } \pm 0.09 \text { (syst) }
$$

The total systematic uncertainty will be higher than the statistical uncertainty. However, there are several experiments at Fermilab and CERN that will have sufficient data to reduce the systematic uncertainty in the cross section measurements which is currently the largest source of systematic error. Combining the statistical and systematic uncertainty in quadrature gives a total error of 0.10 , which is still consistent with CPT conservation. 


\section{Chapter 8}

\section{Conclusions}

The MINOS Far Detector has taken approximately 13 kTon years of data. The total number of events recorded is $4.11 \times 10^{7}$. A series of cuts were imposed to select $17 \times 10^{6}$ muons with reliable timing, tracking, and directionality. Placing the vertex direction cut on these muons separated the neutrino induced candidate muons from the cosmic ray muons. A total of 148 candidate neutrino induced muon events were selected.

The candidate events have been grouped into two event samples. The first sample has all the selected events and is duly called the "all events" sample. The events in the second sample have well measured momentum and identified charge. The sample is called the "high resolution" sample. The subsequent analysis was done using both the event samples.

For the oscillation analysis, the events were additionally separated into low and high momentum events. The ratio of the low to high momentum events was determined for 
the data and the Monte Carlo for both the "all events" and the high resolution samples. The double ratio for both samples shows a significant deviation from unity which indicates neutrino oscillations. The double ratios are,

$$
R_{A}=\frac{R_{\text {low } / \text { high }}^{\text {data }}}{R_{\text {low } / \text { high }}^{M C}}=0.60_{-0.10}^{+0.11}(\text { stat }) \pm 0.08(\text { syst }),
$$

for the "all events" sample, and,

$$
R_{H R}=\frac{R_{\text {low } / \text { high }}^{\text {data }}}{R_{\text {low } / \text { high }}^{M C}}=0.58_{-0.11}^{+0.14}(\text { stat }) \pm 0.05(\text { syst }),
$$

for the high resolution sample. Adding the errors in quadrature, the total error for the "all events" sample is 0.14 , and 0.16 for the high resolution sample. This is a total deviation of $2.9 \sigma$ and $2.6 \sigma$ from unity for the "all events" sample and the "high resolution" sample, respectively.

A combined momentum and zenith angle oscillation fit has been performed using the method of maximum log-likelihood with a grid search in the parameter space of $\Delta m^{2}$ and $\sin ^{2} 2 \theta$. The best fit point for both the event samples occurs at $\Delta m^{2}=1.3 \times 10^{-3}$ $\mathrm{eV}^{2}$, and $\sin ^{2} 2 \theta=1$. This result is compatible with the Super Kamiokande [18] and the MINOS contained analysis [74].

The $68 \%$ and the $90 \%$ contour levels generated for both event samples are smaller than the MINOS contained analysis. Further investigation reveals that the data to Monte Carlo double ratios are lower than the minimum values allowed by the MC. An extended 
likelihood analysis shows that the best fit values for the mixing angle fall in the unphysical region. In order for the Monte Carlo to fit the data, higher values of the mixing angle (greater than one) are required.

The MINOS Far Detector is able to distinguish the charge of the muons. The measured charge of the muons is used to test the rate of the neutrino to the anti-neutrino oscillations by measuring the neutrino induced muon charge ratio. Using the high resolution sample, the $\mu^{+}$to $\mu^{-}$double charge ratio has been determined to be

$$
R_{C P T}=\frac{R_{\mu^{-} / \mu^{+}}^{\text {data }}}{R_{\mu^{-} / \mu^{+}}^{M C}}=0.90_{-0.18}^{+0.24}(\text { stat }) \pm 0.09(\text { syst }) .
$$

With the uncertainties added in quadrature, the CPT double ratio is consistent with unity showing no indication for CPT violation.

An oscillation sensitivity to the current data exposure has also been performed. The contours generated from the sensitivity calculations are comparable to those obtained from the MINOS contained analysis. Using the sensitivity calculation, a 10 year prediction for the likelihood contours is made for the MINOS Far Detector. In addition, a 10 year prediction is made for the $\mathrm{CPT}$ charge ratio assuming that the current double ratio average remains constant. The 1o year CPT charge ratio is

$$
R_{C P T}(10 \text { years })=0.90 \pm 0.05 \text { (stat) } \pm 0.09 \text { (syst) }
$$

The statistical uncertainty is much smaller, and will give a test of the CPT conservation 
at the $5 \%$ level, assuming that new experimental data will reduce the systematic errors below the current values. 


\section{Appendix A}

\section{Neutrino Induced Events List}

The candidate events listed here are from the data taken from August 2003 till April 2006.

The total live time for the data set is 890 days. A total of 148 events have been selected as result of applying all the cuts described in Chapter [6. The tables are separated into high resolution sample and "all events" sample. Note that the events in the "all events" sample are also in the high resolution sample. Therefore, the "all events" table (Table A.5) only lists the events that were excluded from the high resolution sample. The high resolution sample was further divided into four sub-samples: the low momentum $\mu^{-}$events, the low momentum $\mu^{+}$events, the high momentum $\mu^{-}$, and the high momentum $\mu^{+}$events. The information provided in the tables is the measurements used for the oscillation and the CPT conservation analysis. The following information about the events are tabulated: 
- The run number

- The sub run number

- The snarl number

- The measured momentum (GeV)

- The ratio of the measured momentum to the measured error on the momentum

- The negative cosine of the zenith angle

- The inverse beta value

Table A.1: High resolution $\mu^{-}$candidates - (low momentum)

\begin{tabular}{|c|c|c|c|c|c|c|}
\hline \hline Run & Sub Run & Snarl & $p(\mathrm{GeV})$ & $|p / \delta p|$ & $d$ cosy & $1 / \beta$ \\
\hline \hline 20814 & 0 & 1300 & -5.9988 & 9.61168 & 0.966577 & 0.925576 \\
\hline 22165 & 2 & 69462 & -2.2991 & 13.8577 & 0.228626 & 0.987923 \\
\hline 25429 & 1 & 61257 & -5.88973 & 9.98507 & 0.266298 & 0.965988 \\
\hline 25645 & 3 & 111829 & -5.90421 & 32.2988 & 0.323524 & 0.953721 \\
\hline 25683 & 1 & 32242 & -7.32547 & 8.75753 & -0.0187013 & 1.15174 \\
\hline 25773 & 0 & 6897 & -3.28449 & 14.5673 & 0.65407 & 0.897146 \\
\hline & & & \multicolumn{5}{c}{ Continued on next page } \\
\hline
\end{tabular}


Table A.1 - continued from previous page

\begin{tabular}{|c|c|c|c|c|c|c|}
\hline Run & Sub-R & Snarl & $p(\mathrm{GeV})$ & $|p / \delta p|$ & $d$ cosy & $1 / \beta$ \\
\hline \hline 25810 & 1 & 49243 & -11.7885 & 9.16176 & 0.301447 & 0.94602 \\
\hline 25810 & 1 & 60075 & -6.09485 & 14.2873 & 0.204185 & 1.00893 \\
\hline 26383 & 3 & 124483 & -1.37256 & 12.311 & 0.785211 & 0.788593 \\
\hline 27184 & 2 & 96033 & -3.18424 & 24.0409 & 0.452604 & 1.08213 \\
\hline 27615 & 3 & 55040 & -4.7508 & 20.8928 & 0.454226 & 1.04804 \\
\hline 28342 & 1 & 22765 & -5.91486 & 3.03773 & 0.589657 & 1.22771 \\
\hline 29133 & 3 & 40859 & -3.03853 & 8.8961 & -0.056716 & 0.867284 \\
\hline 29170 & 0 & 8142 & -1.84642 & 5.75095 & 0.296531 & 0.685477 \\
\hline 31687 & 3 & 51696 & -9.85329 & 17.9202 & 0.337141 & 0.966075 \\
\hline 31802 & 4 & 69569 & -3.99142 & 22.7428 & 0.24345 & 0.96181 \\
\hline 32227 & 3 & 46394 & -5.23267 & 2.99338 & 0.0225094 & 0.908555 \\
\hline 32481 & 2 & 42692 & -1.92194 & 12.2037 & 0.100652 & 0.816804 \\
\hline 32641 & 22 & 337876 & -5.39089 & 17.8784 & 0.608821 & 0.958918 \\
\hline 32641 & 23 & 360096 & -8.76267 & 14.8021 & 0.370886 & 0.953608 \\
\hline 32788 & 22 & 332009 & -1.9235 & 12.0154 & 0.0386262 & 1.16366 \\
\hline 32805 & 12 & 178610 & -11.9537 & 7.33208 & 0.48164 & 1.01572 \\
\hline 33035 & 4 & 68870 & -2.88183 & 18.3876 & 0.700755 & 1.08812 \\
\hline & & & & & Continued on next page \\
\hline
\end{tabular}


Table A.1 - continued from previous page

\begin{tabular}{|c|c|c|c|c|c|c|}
\hline Run & Sub-R & Snarl & $p(\mathrm{GeV})$ & $|p / \delta p|$ & $d$ cosy & $1 / \beta$ \\
\hline \hline 33083 & 10 & 150964 & -2.99622 & 9.9328 & -0.0145764 & 0.967116 \\
\hline 33567 & 6 & 72699 & -2.62636 & 14.9483 & 0.563293 & 0.793547 \\
\hline 33735 & 3 & 37195 & -5.58586 & 32.5924 & 0.419654 & 1.02974 \\
\hline \hline
\end{tabular}

Table A.2: High resolution $\mu^{+}$candidates - (low momentum)

\begin{tabular}{|c|c|c|c|c|c|c|}
\hline \hline Run & Sub Run & Snarl & $p(\mathrm{GeV})$ & $|p / \delta p|$ & $d$ cosy & $1 / \beta$ \\
\hline \hline 18761 & 0 & 65898 & 3.85886 & 4.69158 & 0.318859 & 0.639345 \\
\hline 19292 & 0 & 49901 & 5.31474 & 21.1829 & 0.886147 & 1.13546 \\
\hline 21361 & 0 & 12693 & 8.40752 & 29.8779 & 0.378737 & 0.975523 \\
\hline 23692 & 4 & 85806 & 1.95169 & 8.19474 & 0.685616 & 0.801387 \\
\hline 24168 & 2 & 85867 & 2.16463 & 7.56659 & 0.577993 & 1.01708 \\
\hline 24189 & 1 & 55106 & 9.82001 & 3.96966 & 0.318048 & 0.911361 \\
\hline 27488 & 0 & 6258 & 1.65983 & 15.0128 & 0.135509 & 0.932451 \\
\hline 28034 & 7 & 582730 & 1.66109 & 5.01256 & 0.210598 & 1.12694 \\
\hline 28615 & 6 & 78923 & 3.74524 & 10.109 & 0.165767 & 1.12088 \\
\hline 29063 & 4 & 60101 & 5.95155 & 4.69641 & 0.312774 & 0.966271 \\
\hline & & & & & Continued on next page \\
\hline
\end{tabular}


Table A.2 - continued from previous page

\begin{tabular}{|c|c|c|c|c|c|c|}
\hline Run & Sub-R & Snarl & $p(\mathrm{GeV})$ & $|p / \delta p|$ & $d$ cosy & $1 / \beta$ \\
\hline \hline 30680 & 2 & 40731 & 3.09195 & 3.55356 & 0.608383 & 0.919725 \\
\hline 31201 & 3 & 53906 & 3.1524 & 20.2504 & 0.102219 & 1.05638 \\
\hline 24189 & 1 & 55106 & 9.82001 & 3.96966 & 0.318048 & 0.911361 \\
\hline 27488 & 0 & 6258 & 1.65983 & 15.0128 & 0.135509 & 0.932451 \\
\hline 28034 & 7 & 582730 & 1.66109 & 5.01256 & 0.210598 & 1.12694 \\
\hline 28615 & 6 & 78923 & 3.74524 & 10.109 & 0.165767 & 1.12088 \\
\hline 29063 & 4 & 60101 & 5.95155 & 4.69641 & 0.312774 & 0.966271 \\
\hline 30680 & 2 & 40731 & 3.09195 & 3.55356 & 0.608383 & 0.919725 \\
\hline 31201 & 3 & 53906 & 3.1524 & 20.2504 & 0.102219 & 1.05638 \\
\hline 32865 & 4 & 52883 & 10.5715 & 16.9192 & 0.214689 & 1.00764 \\
\hline 33023 & 17 & 248263 & 7.0183 & 9.7858 & -0.00764896 & 0.900451 \\
\hline 33606 & 22 & 266453 & 2.02579 & 8.10476 & 0.577408 & 0.766492 \\
\hline 34583 & 11 & 112418 & 7.61578 & 11.2659 & 0.902316 & 1.0173 \\
\hline \hline
\end{tabular}


Table A.3: High resolution $\mu^{-}$candidates - (high momentum)

\begin{tabular}{|c|c|c|c|c|c|c|}
\hline \hline Run & Sub Run & Snarl & $p(\mathrm{GeV})$ & $|p / \delta p|$ & $d$ cosy & $1 / \beta$ \\
\hline \hline 18900 & 0 & 94973 & -72.7595 & 14.9198 & -0.0370652 & 0.996037 \\
\hline 20966 & 0 & 27391 & -72.6889 & 3.37475 & 0.0880242 & 1.03977 \\
\hline 22213 & 6 & 178971 & -19.4348 & 4.55112 & 0.185003 & 1.01914 \\
\hline 22650 & 6 & 124339 & -13.2473 & 6.02443 & 0.0577239 & 0.97972 \\
\hline 22672 & 2 & 46426 & -18.4017 & 3.34868 & 0.0616845 & 0.992162 \\
\hline 24767 & 0 & 22875 & -212.576 & 6.6906 & 0.177064 & 0.991137 \\
\hline 25355 & 0 & 12396 & -19.6423 & 6.11003 & 0.314998 & 0.963284 \\
\hline 25651 & 0 & 16646 & -15.2176 & 7.13683 & 0.456973 & 1.03238 \\
\hline 25671 & 2 & 78546 & -37.3955 & 3.19339 & 0.700928 & 0.944115 \\
\hline 26996 & 1 & 42485 & -15.4062 & 7.72302 & 0.112299 & 0.940847 \\
\hline 27514 & 6 & 99919 & -15.9358 & 6.28672 & 0.826752 & 1.02775 \\
\hline 28357 & 4 & 56453 & -21.8213 & 4.31902 & 0.847959 & 0.990469 \\
\hline 29083 & 6 & 83732 & -20.6397 & 2.707 & 0.916501 & 1.01224 \\
\hline 29359 & 3 & 36525 & -15.3862 & 6.97226 & 0.098357 & 0.987146 \\
\hline 30374 & 0 & 9962 & -97.2214 & 11.4085 & 0.0151494 & 0.991166 \\
\hline 31745 & 4 & 76534 & -52.2143 & 12.6968 & 0.424383 & 0.998183 \\
\hline & & & & & Continued on next page \\
\hline
\end{tabular}


Table A.3 - continued from previous page

\begin{tabular}{|c|c|c|c|c|c|c|}
\hline Run & Sub-R & Snarl & $p(\mathrm{GeV})$ & $|p / \delta p|$ & $d$ cosy & $1 / \beta$ \\
\hline \hline 31846 & 2 & 43413 & -38.8592 & 2.69864 & 0.406836 & 0.923132 \\
\hline 32669 & 2 & 41218 & -22.2091 & 9.2827 & 0.402301 & 0.969244 \\
\hline 32734 & 15 & 234184 & -19.2952 & 12.9242 & 0.618319 & 1.03443 \\
\hline 32782 & 1 & 22270 & -14.2553 & 9.00192 & 0.765302 & 0.956344 \\
\hline 32907 & 10 & 155494 & -74.6952 & 2.74864 & 0.49198 & 1.0494 \\
\hline 33452 & 17 & 227400 & -12.2077 & 4.37254 & 0.398848 & 1.04048 \\
\hline 33455 & 6 & 75713 & -13.6942 & 6.98313 & 0.589172 & 1.06713 \\
\hline 33514 & 11 & 140532 & -12.3448 & 16.7107 & 0.579748 & 0.980978 \\
\hline 34641 & 2 & 23869 & -13.9528 & 4.16833 & 0.924832 & 0.938563 \\
\hline 34747 & 21 & 228199 & -111.873 & 4.38573 & 0.382486 & 0.960866 \\
\hline \hline
\end{tabular}


Table A.4: High resolution $\mu^{+}$candidates - (high momentum)

\begin{tabular}{|c|c|c|c|c|c|c|}
\hline \hline Run & Sub Run & Snarl & $p(\mathrm{GeV})$ & $|p / \delta p|$ & $d$ cosy & $1 / \beta$ \\
\hline \hline 18324 & 0 & 72689 & 19.9951 & 7.64104 & 0.539408 & 1.00996 \\
\hline 20012 & 2 & 63105 & 18.1344 & 3.76713 & 0.390641 & 0.966593 \\
\hline 20958 & 0 & 9755 & 13.1441 & 9.80748 & 0.644354 & 0.984023 \\
\hline 22216 & 0 & 32673 & 49.0114 & 12.5403 & -0.0537556 & 1.0038 \\
\hline 22351 & 1 & 22703 & 12.5693 & 17.1595 & 0.589503 & 0.966928 \\
\hline 25752 & 0 & 17348 & 27.7415 & 16.9171 & 0.448457 & 0.984148 \\
\hline 27790 & 3 & 36378 & 27.79 & 15.2272 & 0.0763574 & 0.990251 \\
\hline 29449 & 7 & 92140 & 13.9758 & 59.5561 & 0.180969 & 0.984056 \\
\hline 30106 & 0 & 588 & 12.5111 & 17.078 & 0.0992326 & 0.959633 \\
\hline 31291 & 7 & 100788 & 49.3298 & 2.99509 & 0.973625 & 0.887345 \\
\hline 31357 & 3 & 53122 & 26.3375 & 18.2346 & 0.245895 & 0.988247 \\
\hline 33265 & 5 & 76305 & 25.9633 & 2.76517 & -0.0323399 & 1.04549 \\
\hline 33280 & 3 & 38414 & 14.6086 & 4.41119 & -0.0597384 & 1.02418 \\
\hline 33412 & 16 & 194503 & 15.1696 & 22.8195 & 0.383801 & 1.00267 \\
\hline \hline
\end{tabular}


Table A.5: "All Events" candidates

\begin{tabular}{|c|c|c|c|c|c|c|}
\hline \hline Run & Sub Run & Snarl & $p(\mathrm{GeV})$ & $|p / \delta p|$ & $d$ cosy & $1 / \beta$ \\
\hline \hline 18890 & 0 & 67171 & -36.3979 & 0.921229 & 0.825638 & 1.13949 \\
\hline 19025 & 0 & 18948 & -55.9038 & 2.29788 & 0.0780862 & 0.955372 \\
\hline 19302 & 0 & 14996 & 29388.3 & 0.00317303 & 0.71759 & 1.01564 \\
\hline 19302 & 0 & 40691 & -66.7113 & 2.10005 & 0.156055 & 1.00008 \\
\hline 19334 & 0 & 84654 & 273.798 & 0.282782 & 0.811584 & 1.05855 \\
\hline 20566 & 0 & 63713 & -118.555 & 0.68784 & 0.882523 & 0.935358 \\
\hline 20941 & 0 & 40478 & 29.6598 & 2.40985 & 0.347473 & 0.979244 \\
\hline 21361 & 0 & 8220 & 29.2952 & 0.681593 & 0.726243 & 1.11259 \\
\hline 21398 & 1 & 51585 & 110.779 & 0.0734019 & 0.519439 & 1.05868 \\
\hline 21485 & 1 & 35404 & -25.1436 & 1.73318 & 0.28515 & 0.919817 \\
\hline 21845 & 0 & 463 & -259.889 & 0.822636 & 0.752325 & 1.01404 \\
\hline 21853 & 1 & 22982 & 71.3277 & 0.746124 & 0.868767 & 1.10461 \\
\hline 21865 & 3 & 84239 & -185.232 & 0.287846 & 0.706926 & 0.994686 \\
\hline 22342 & 0 & 9399 & 92.6259 & 1.7681 & 0.746274 & 1.00505 \\
\hline 22566 & 4 & 95838 & 20.9821 & 1.28913 & 0.168725 & 1.1071 \\
\hline 22734 & 4 & 94200 & 50.3381 & 0.544989 & 0.35828 & 0.97436 \\
\hline & & & & & Continued on next page \\
\hline
\end{tabular}


Table A.5 - continued from previous page

\begin{tabular}{|c|c|c|c|c|c|c|}
\hline Run & Sub-R & Snarl & $p(\mathrm{GeV})$ & $|p / \delta p|$ & $d$ cosy & $1 / \beta$ \\
\hline 22968 & 2 & 39057 & -2593.76 & 0.630455 & 0.0961464 & 0.99092 \\
\hline 23087 & 7 & 130718 & -39.5067 & 0.284018 & 0.541817 & 0.848557 \\
\hline 23090 & 4 & 80549 & 34.9057 & 1.63549 & 0.935527 & 0.865162 \\
\hline 23285 & 1 & 23840 & -129.583 & 2.22861 & 0.0421867 & 1.0407 \\
\hline 24884 & 0 & 15375 & 617.462 & 0.258882 & 0.93553 & 1.0057 \\
\hline 25601 & 2 & 77297 & -364.384 & 1.575 & 0.151828 & 0.977477 \\
\hline 25677 & 3 & 103707 & -41.6864 & 2.33425 & 0.811445 & 1.12377 \\
\hline 26014 & 3 & 135812 & 295.919 & 0.159197 & 0.596902 & 0.935371 \\
\hline 26307 & 0 & 12411 & 14.6745 & 1.89163 & 0.947544 & 1.08106 \\
\hline 26389 & 0 & 342 & -20236.3 & 0.00518258 & 0.727239 & 0.92968 \\
\hline 26866 & 1 & 62232 & 104.688 & 1.48785 & -0.00264404 & 0.96915 \\
\hline 26947 & 2 & 63690 & 153.255 & 0.997851 & -0.0151518 & 0.975113 \\
\hline 27199 & 3 & 105617 & 30.8413 & 0.892456 & 0.331561 & 0.983282 \\
\hline 28267 & 4 & 56432 & -52411.4 & 0.0010762 & 0.473527 & 1.06107 \\
\hline 28454 & 7 & 85477 & -61.9919 & 1.81495 & 0.885953 & 0.918795 \\
\hline 28550 & 6 & 69552 & 65.6118 & 1.14395 & 0.0602388 & 0.979246 \\
\hline 28621 & 2 & 27682 & 37.0483 & 1.15595 & 0.854109 & 0.959315 \\
\hline & & & & & Continued on 0 ext page \\
\hline
\end{tabular}


Table A.5 - continued from previous page

\begin{tabular}{|c|c|c|c|c|c|c|}
\hline Run & Sub-R & Snarl & $p(\mathrm{GeV})$ & $|p / \delta p|$ & $d$ cosy & $1 / \beta$ \\
\hline 28999 & 7 & 86753 & 69.9954 & 0.969598 & 0.314305 & 0.986502 \\
\hline 29020 & 0 & 5033 & -226.759 & 2.02298 & 0.314241 & 0.98959 \\
\hline 29063 & 0 & 5496 & -83.8172 & 0.993146 & 0.115184 & 0.99214 \\
\hline 29310 & 7 & 93754 & -20.1454 & 1.32181 & 0.510563 & 1.12381 \\
\hline 29474 & 3 & 39063 & 26.5157 & 1.01342 & -0.0497505 & 0.98412 \\
\hline 29475 & 5 & 69571 & -530.108 & 0.900767 & 0.486863 & 0.983109 \\
\hline 30613 & 7 & 91524 & -131.293 & 1.43265 & 0.227652 & 1.03603 \\
\hline 30643 & 2 & 26558 & 1910.24 & 0.0495468 & 0.102923 & 0.922436 \\
\hline 30681 & 3 & 53952 & -57.5315 & 2.36445 & 0.0117192 & 0.99135 \\
\hline 31250 & 2 & 32275 & -55.5078 & 0.285312 & 0.617356 & 1.01781 \\
\hline 31276 & 2 & 38335 & 62.5678 & 0.178171 & 0.0184227 & 1.06556 \\
\hline 31674 & 1 & 28703 & 7.49818 & 1.32316 & 0.248544 & 1.05302 \\
\hline 31687 & 6 & 104086 & 55.3193 & 1.23458 & 0.495018 & 0.912035 \\
\hline 31822 & 6 & 104611 & 153.514 & 0.882906 & 0.276461 & 0.979215 \\
\hline 31912 & 7 & 113565 & -622.69 & 0.198526 & 0.167118 & 1.08169 \\
\hline 32127 & 2 & 37518 & -62 & 0.687993 & 0.768917 & 1.00513 \\
\hline 32499 & 5 & 81213 & 147.452 & 2.2644 & 0.152642 & 0.997655 \\
\hline & & & & & Continued on 0 next page \\
\hline
\end{tabular}


Table A.5 - continued from previous page

\begin{tabular}{|c|c|c|c|c|c|c|}
\hline Run & Sub-R & Snarl & $p(\mathrm{GeV})$ & $|p / \delta p|$ & $d$ cosy & $1 / \beta$ \\
\hline 32614 & 16 & 247402 & -41.2279 & 1.53393 & 0.439973 & 1.03353 \\
\hline 32623 & 19 & 288190 & -210.126 & 1.30517 & -0.0278136 & 1.04282 \\
\hline 32669 & 6 & 93650 & -156.689 & 0.70487 & 0.159134 & 0.978081 \\
\hline 32817 & 0 & 4451 & -91.564 & 0.607282 & 0.870441 & 0.990187 \\
\hline 32868 & 12 & 150167 & 5.52709 & 1.78373 & 0.556634 & 0.594642 \\
\hline 32962 & 3 & 43222 & 13.8705 & 1.26406 & 0.282061 & 1.379 \\
\hline 33020 & 4 & 67342 & 95.076 & 0.341241 & 0.610338 & 0.942548 \\
\hline 33026 & 12 & 171030 & -99.7433 & 0.144477 & 0.340318 & 0.875263 \\
\hline 33086 & 3 & 49875 & -324.705 & 0.320958 & 0.240876 & 0.935152 \\
\hline 33156 & 11 & 149275 & 13.4516 & 2.28166 & 0.671998 & 1.13605 \\
\hline 33253 & 5 & 67019 & 15.4618 & 1.65945 & 0.946259 & 0.989992 \\
\hline 33489 & 10 & 130632 & 316.067 & 0.107244 & 0.0114983 & 1.00643 \\
\hline 33542 & 2 & 30297 & 45.1566 & 0.717283 & 0.226286 & 1.07508 \\
\hline 33542 & 20 & 259440 & -34.119 & 0.633779 & 0.41427 & 0.808364 \\
\hline 33824 & 17 & 177037 & 189.748 & 1.57182 & 0.475243 & 0.983786 \\
\hline 34744 & 7 & 82431 & -8.03719 & 1.12381 & -0.0558481 & 0.641297 \\
\hline \hline
\end{tabular}




\section{References}

[1] Z. Maki, M. Nakagawa, and S. Sakata, Prog. Theor. Phys. 28, (1962) 870.

[2] K. Hagiwara, et al., Physical Review, D393, 2002.

[3] S. P. Mikheyev, A. Yu. Smirnov, Sov. J. Nucl. Phys., 42, (1985) 913.

[4] L. Wolfenstein, Phys. Rev. D17, 2369 (1978).

[5] L. L. Chau and W. Y. Keung, Phys. Rev. Lett. 53, 1802 (1984).

[6] M. Apollonio, Phys. Lett. B466, 415 (1999).

[7] F. Boehm, Phys. Rev. D62, 072002 (2000).

[8] S. T. Petcov, Phys. Lett. B434, 321 (1998).

[9] S. T. Petcov, Nucl. Phys. B (Proc. Suppl.) 77, 93 (1999).

[10] Sergio Palomares-Ruiz, S. T. Petcov hep-ph/0406096.

[11] R. Becker-Szendy et al., Nucl. Phys. Proc. Suppl. A324, (1993) 363. 
[12] R. Becker-Szendy et al., Phys. Rev. D46, (1992) 3720.

[13] K. S. Hirata et al., Phys. Lett. B280, (1992) 146.

[14] Battistoni G. et al.[The NUSEX Collaboration], The NUSEX Detector, Nucl. Instr. Meth. A245, (1986) 277.

[15] M. Aglietta et al. [The NUSEX Collaboration], Europhys. Lett. 8, (1989) 611.

[16] K. Daum et al. [Frejus Collaboration], Z. Phys. C 66, (1995) 417.

[17] Y. Ashie et al., Phys. Rev. D71, (2005) 112005

[18] Y. Ashie et al., Phys. Rev. Lett. 99, (2004) 101801.

[19] Y. Fukuda et al. Phys. Lett. B467, (1999) 185.

[20] Y. Fukuda et al., Phys. Lett. B433, (1998) 9.

[21] W. Allison et al., Phys. Lett. B449, (1999) 137.

[22] W. Allison et al., Nucl. Instr. Meth A376 (1996) 36.

[23] W. Allison et al., Phys. Lett. B391 (1997) 491.

[24] M. Sanchez et al., Phys. Rev. D68 (2003) 113004.

[25] G. Feldman and R. Cousins, Phys. Rev. D57 (1998) 3873.

[26] V. D. Barger, J. G. Learned, S. Pakvasa, and T. J.Weiler, Phys. Rev. Lett. 82, (1999) 2640. 
[27] Y. Grossman and M. P. Worah (1998), hep-ph/9807511

[28] W. A. Mann et al., Nucl. Phys. Proc. Suppl. 91, (2000) 134.

[29] S. Ahn et al., Phys. Lett. B511, (2001) 178.

[30] E. Aliu et al., Phys. Rev. Lett., 94, (2005) 081802.

[31] S. Kopp, (2005) physics/0508001.

[32] B. Rebel, PhD thesis, Indiana University, (2004).

[33] D. A. Petyt, PhD thesis, University of Oxford, (1998).

[34] (the MINOS Collaboration) P. Adamson et al., Magnetic Field Maps for the Near and Far MINOS Detector. MINOS-doc-1633-v1 (MINOS technical note), 2006.

[35] B. Becker, PhD Thesis, University of Minnesota, 2006.

[36] C. B. Smith, PhD Thesis, University College London, 2002.

[37] J. Simpson, Ann. Rev. Nucl. Part. Sci., 33, 323 (2004).

[38] J. Wang, Astrophys. J., 564, 244 (2002).

[39] M. Boezio et. al. Astrophys. J. 518, 457 (1999).

[40] W. Menn et. al. Astrophys. J. 533, 281 (2000).

[41] J. Alcaraz, et. al. Phys. Lett. B490, 27 (2000). 
[42] D. Bird et. al. Astrophys. J., 424, 491 (1994).

[43] J. Kremer et. al. Phys. Rev. Lett. 83, 4241 (1999).

[44] M. Motoki et. al. Astropart. Phys. 19, 113 (2003).

[45] P Archard et. al. Phys. Lett B598, 15 (2004).

[46] T. Gaisser (1990), Cambridge, UK, Univ. Pr. 279 p.

[47] S. Kasahara, PhD Thesis, University of Minnesota, (1997).

[48] R. Hatcher, NuMI-NOTE-SIM-0479 (MINOS technical Note), (1999).

[49] S. Giani et al., GEANT detector description and simulation tool, CERN Program Library Long Writeup W5013 (1994).

[50] N Tagg, DetSim and Photon Transport, http://wwwnumi.fnal.gov/offline_software/srt_public_context/webdocs/detsim/detsim_documentation.html.

[51] A. Blake, PhD Thesis, Cambridge University, (2005).

[52] T. K. Gaissr and M. Honda Ann. Rev. Nucl. Part. Sci, 52, 153 (2002).

[53] J. Alcarez, et al., Phys. Lett B490, 27 (2000).

[54] T. Sanuki, et al., Astrophys J., 555, 1135 (2000). 
[55] W. Webber, R. Golden, and S. Stephens, Proc. 20th Int. Cosmic Ray Conf. 1, 325 (1978).

[56] E-S Seo, et al., Astrophys J., 378, 763 (1991).

[57] P. Papini, et al., 23rd Int. Cosmic Ray Conf. 1, 579 (1993).

[58] M. Boezio, et al., Astrophys J., 518, 457 (1999).

[59] W. Menn, et al., Astrophys J., 533, 281 (2000).

[60] M. Ryan, F. Ormes, and V. Balasubrahmanyan, Phys. Rev. Lett. 28, 958; erratum 281497 (1972).

[61] K. Asakimori, et al., Ap. J., 502, 278 (1998).

[62] I. Ivanenko, et al., Proc. 23rd Int. Cosmic Ray Conf. 217 (1993).

[63] A. Apanasenko, et al., Astropart. Phys. 16, 13 (2001).

[64] Y. Kawamura, et al., Phys. Rev. D40, 729 (1989).

[65] P. Lipari, et al., Phys. Rev. Lett. 47, 4384 (1994).

[66] D. Farlane, et al., Z. Phys. C, 1 (1984).

[67] N. Baker, et al., Phys. Rev. D25, 617 (1982).

[68] S. Barish, et al., Phys. Rev. D19, 2521 (1979).

[69] S. Barish, et al., Phys. Rev. D16, 3103 (1977). 
[70] D. Casper Nucl. Phys. Proc. Suppl. 112), 161 (2002).

[71] V. Agrawal, T. Gaisser, P. Lipari, and T. Stanev, Phys. Rev. D53, 1314 (1996).

[72] M. Gluc, E. Reya, and A Vogt, Z. Phys. C 67, 433 (1995).

[73] K. Hagiwara, et al. Review of Particle Physics, Physical Review D66, 2002.

[74] P. Adamson et al., (the MINOS Collaboration) Phys. Rev. D 73, 072002 (2006)

[75] R. Lee, Event Reconstruction in the Near Detector, NuMI-Note-COMP-917 (Internal Pulblication).

[76] G. D. Barr et al. Submitted to Phys. Rev. D (2006) astro-ph/0611266.

[77] P. S. Auchincloss et al., Z. Phys. C48, (1990) 411.

[78] S. Eidelman et al., Phys. Lett. B592, (2004) 15.

[79] N. Gehrels, Astrophys. J. 303, (1986) 411.

[80] W. W. Allison, et al. Phys. Lett. B391, (1997) 491. 